\title{
Development and Validation of a Tire Model for a Real Time Simulation of a Helicopter Traversing and Manoeuvring on a Ship Flight Deck
}

\author{
by \\ Jason P. Tremblay, B.Eng. \\ Carleton University \\ A thesis submitted to \\ the Faculty of Graduate Studies and Research \\ in partial fulfillment of \\ the requirements for the degree of \\ Masters of Applied Science \\ Ottawa-Carleton Institute for \\ Mechanical and Aerospace Engineering \\ Department of \\ Mechanical and Aerospace Engineering \\ Carleton University \\ Ottawa, Ontario \\ January 12, 2007 \\ (C) Copyright \\ 2007 - Jason P. Tremblay
}




$\begin{array}{ll}\begin{array}{l}\text { Library and } \\ \text { Archives Canada }\end{array} & \begin{array}{l}\text { Bibliothèque et } \\ \text { Archives Canada }\end{array} \\ \begin{array}{l}\text { Published Heritage } \\ \text { Branch }\end{array} & \begin{array}{l}\text { Direction du } \\ \text { Patrimoine de l'édition }\end{array} \\ \begin{array}{l}\text { 395 Wellington Street } \\ \text { Ottawa ON K1A 0N4 }\end{array} & \begin{array}{l}\text { 395, rue Wellington } \\ \text { Ottana ON K1A ON4 } \\ \text { Canada Oa }\end{array}\end{array}$

Your file Votre référence ISBN: 978-0-494-27004-2 Ourfile Notre référence ISBN: 978-0-494-27004-2

NOTICE:

The author has granted a nonexclusive license allowing Library and Archives Canada to reproduce, publish, archive, preserve, conserve, communicate to the public by telecommunication or on the Internet, loan, distribute and sell theses worldwide, for commercial or noncommercial purposes, in microform, paper, electronic and/or any other formats.

The author retains copyright ownership and moral rights in this thesis. Neither the thesis nor substantial extracts from it may be printed or otherwise reproduced without the author's permission.
AVIS:

L'auteur a accordé une licence non exclusive permettant à la Bibliothèque et Archives Canada de reproduire, publier, archiver, sauvegarder, conserver, transmettre au public par télécommunication ou par l'Internet, prêter, distribuer et vendre des thèses partout dans le monde, à des fins commerciales ou autres, sur support microforme, papier, électronique et/ou autres formats.

L'auteur conserve la propriété du droit d'auteur et des droits moraux qui protège cette thèse. $\mathrm{Ni}$ la thèse ni des extraits substantiels de celle-ci ne doivent être imprimés ou autrement reproduits sans son autorisation.
In compliance with the Canadian

Privacy Act some supporting forms may have been removed from this thesis.

While these forms may be included in the document page count, their removal does not represent any loss of content from the thesis.
Conformément à la loi canadienne sur la protection de la vie privée, quelques formulaires secondaires ont été enlevés de cette thèse.

Bien que ces formulaires aient inclus dans la pagination, il n'y aura aucun contenu manquant. 


\section{Abstract}

A transient tire model was developed to improve a simulation program, HeliMan, used to model the manoeuvring and traversing of a maritime helicopter on a ship flight deck. The nonlinear single contact patch transient tire model is used to simulate the tire behaviour. The nonlinear differential equation of the transient tire model is solved by obtaining the instantaneous relaxation length of the tire through the use of the steady state Magic Formula and Similarity Method tire models. The Magic Formula model was developed by matching empirical tire test results, collected as part of this project, to the curve fits of the model. The Similarity Method uses the Magic Formula model to produce a set of nominal conditions that are then adapted to changes made by normal force or frictional coefficient acting on the tire.

Tire tests were conducted using Carleton University's tire testing facility, which was retrofitted in order to test aircraft tires on the surface of a non-skid ship deck. Forces acting on a particular representative aircraft tire were collected as slip angle, camber inclination, and normal force was varied. The resulting tire model was adapted to match the performance of a Seahawk helicopter manoeuvring and traversing on a ship flight deck under the control of the Aircraft/Ship Integrated Secure and Traverse (ASIST) system. The simulation was validated by comparing the results to those obtained through full-scale validation tests. The validation tests consisted of comparing simulation results with data collected using a Dead Load Test Vehicle (DLTV) simulating a helicopter, undergoing several manoeuvres under the control of the ASIST aircraft handling system.

The tire model improved the tire force generation of the HeliMan simulation and captured the underlying dynamics of the helicopter manoeuvring and traversing on a ship flight deck. A further key benefit of the tire model is its inherent flexibility in terms of applying it to different aircraft tires without the necessity for extensive tire 
testing each time a different tire design must be simulated. 


\section{Acknowledgements}

I would like to extend my gratitude to my thesis supervisor, Dr. Robert Langlois. Rob has made my time as a graduate student truly enjoyable. He has always been more than willing to lend both his time and experience whenever it was required. I wish him the best of luck in his career and in his research.

I would like to give a warm thank you to the staff of Carleton's Mechanical and Aerospace Department. The office was always helpful and the technical support staff was always willing to lend a helping hand. I would like to give special mention to Fred Barrett, and Steve Truttmann who both provided a great amount of assistance and equipment for my work in the tire test laboratory. I would also like to extend my thanks to Jim Sliwka and Alex Proctor for their guidance in the machine shop.

Thanks to all my colleagues in the graduate department and in the dynamics research team. You have made my time at Carleton truly enjoyable and full of memories.

Finally, I would like to thank both my mother and my father for all their love and support. 


\title{
List of Symbols
}

\author{
Subscripts: \\ $X, Y, Z \quad$ ground reference plane \\ c contact point centre with no slip reference \\ $i \quad$ time step \\ $\hat{i}, \hat{j}, \hat{k} \quad$ direction vectors relative to the ground \\ o nominal condition \\ $r \quad$ variables pertaining to residual moment \\ $s \quad$ contact point centre with slip reference \\ $t \quad$ variables pertaining to the pneumatic trail \\ $x, y, z \quad$ reference plane of the tire contact patch centre with the \\ $z$ component pointing downward, normal to the ground \\ $\alpha \quad$ variable affected by the lateral or side slip \\ $\gamma \quad$ variable affected by tire camber \\ $\kappa \quad$ variable affected by the longitudinal slip \\ $\varphi \quad$ variable affected by the turn slip
}

Superscripts:

transient variation of the variable

Variables:

$B \quad$ Magic Formula stiffness parameter

C Magic Formula shape factor

$C_{\text {bend }} \quad$ bending stiffness of the string model contact patch

$C_{c} \quad$ constant used in the contact patch string model 


\begin{tabular}{|c|c|}
\hline$C_{\text {lat }}$ & lateral stiffness of the tire's string model contact patch \\
\hline$C_{F x}$ & longitudinal stiffness of the tire \\
\hline$C_{F y}$ & lateral stiffness of the tire \\
\hline$C_{F \alpha}$ & tire cornering stiffness \\
\hline$C_{F \gamma}$ & lateral stiffness due to camber \\
\hline$C_{F \kappa}$ & tire stiffness due to longitudinal slip \\
\hline$C_{M \alpha}$ & angular stiffness due to side slip \\
\hline$C_{M \gamma}$ & angular stiffness due to camber inclination \\
\hline$C_{s}$ & constant used in the tire contact patch string model \\
\hline$C_{\text {yaw }}$ & yaw stiffness of the string model contact patch \\
\hline$D$ & Magic Formula peak value parameter \\
\hline E & Magic Formula curvature parameter \\
\hline$H$ & Magic Formula additional curvature parameter \\
\hline$M_{z}$ & aligning torque \\
\hline$M_{z r}$ & residual torque component of the aligning torque \\
\hline$M_{z \varphi \infty}$ & aligning torque of a tire under pure rotation about \\
\hline & its vertical axis \\
\hline$P$ & Similarity Method parameters involving normal force \\
\hline$S_{H}$ & Magic Formula horizontal curve shift parameter \\
\hline$S_{V}$ & Magic Formula vertical curve shift parameter \\
\hline$V_{r}$ & longitudinal velocity of the tire when free rolling \\
\hline$a$ & half length of the tire contact patch \\
\hline$b$ & half width of the tire contact patch \\
\hline$c$ & constant parameters involved in the Magic Formula and Similarity \\
\hline & Method tire models \\
\hline & stiffness of the tire contact patch \\
\hline
\end{tabular}




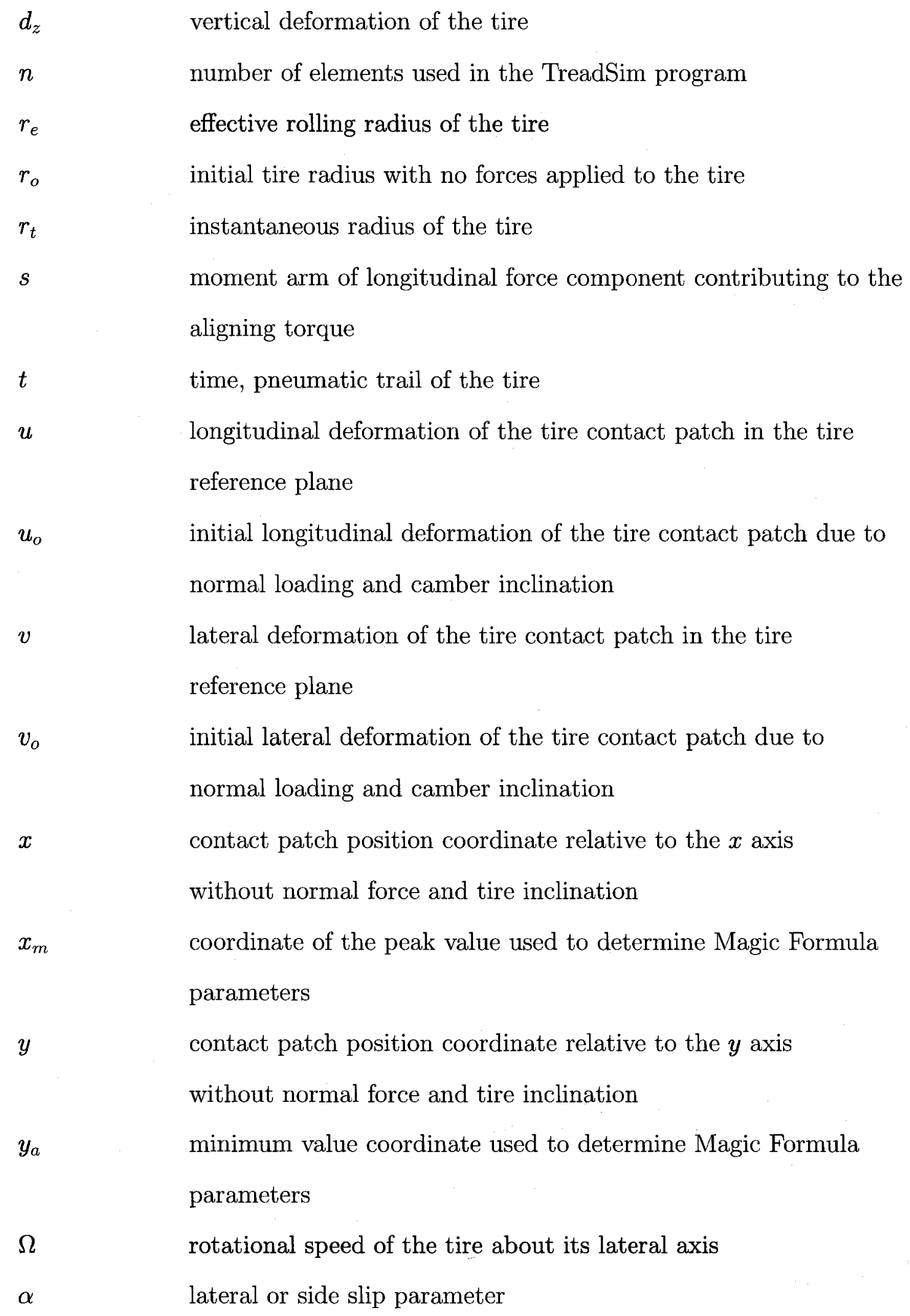




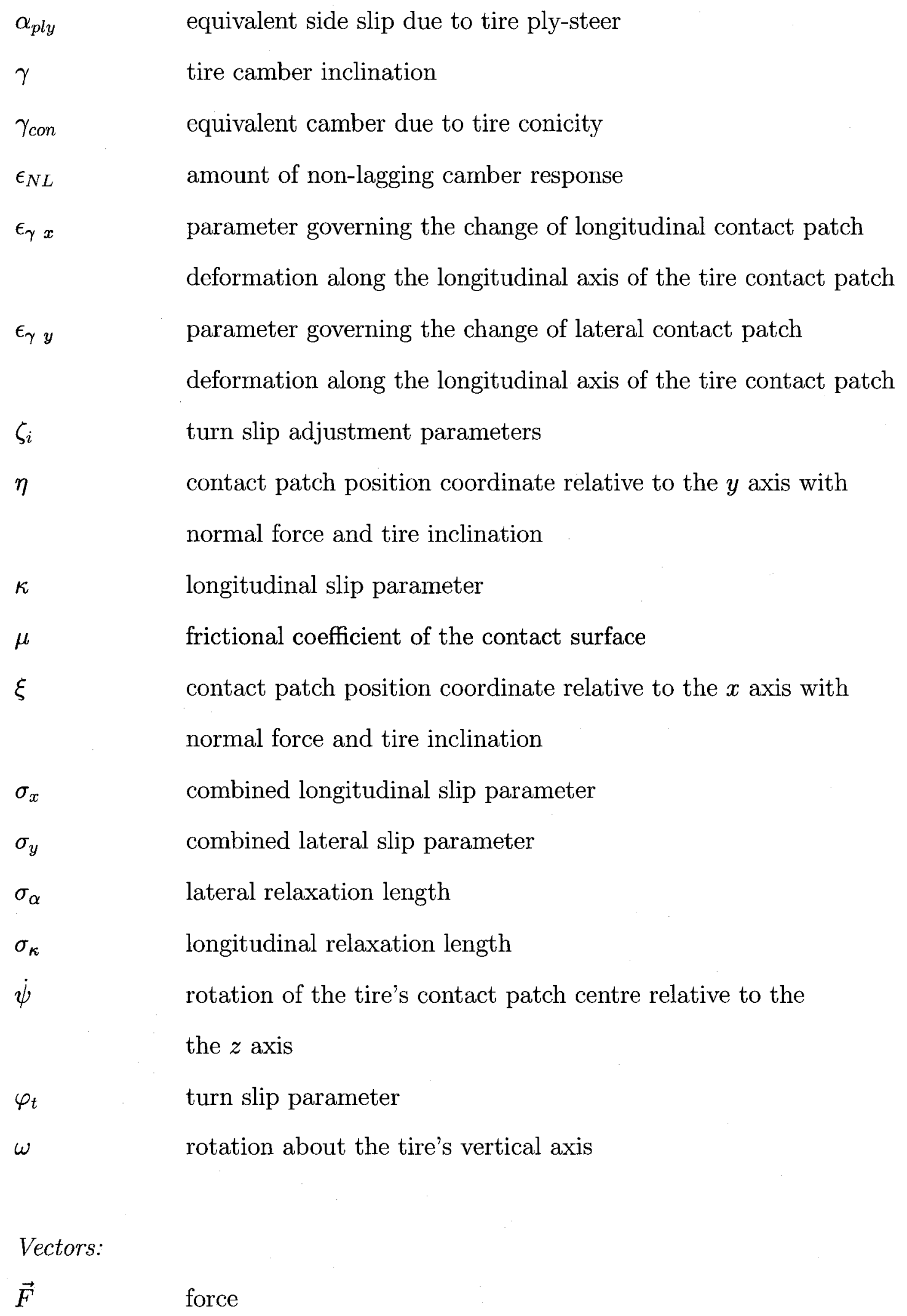


$\vec{P}$

$\vec{Q}$

$\vec{V}$

$\vec{e}$

$\vec{g}$

$\vec{q}$

$\vec{s}$

velocity of a point on the contact patch relative to the ground

velocity of a point on the contact patch relative to the

contact patch centre

velocity

deformation vector of a point on the contact patch

sliding velocity of a point on the tire's contact patch

shear or pressure of a point on the tire's contact patch

displacement of a point on the tire contact patch without

the influence of tire slip 


\section{Contents}

Acceptance $\quad$ ii

Abstract $\quad$ iii

Acknowledgements $\quad$ v

List of Symbols $\quad$ vi

Contents $\quad$ xi

List of Figures xiv

List of Tables $\quad$ xx

1 Introduction $\quad 1$

1.1 Motivation ........................... 1

1.2 Tire Simulation . . . . . . . . . . . . . . . . 3

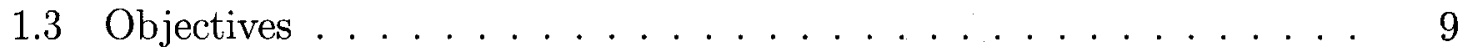

1.4 Thesis Overview . . . . . . . . . . . . . . . . 10

2 Tire Model Theory 12

2.1 Tire Input Quantities . . . . . . . . . . . . . . . . . . 12

2.2 Computational Modelling . . . . . . . . . . . . . . . . 19

xi 
2.2.1 Motion of the Tire Contact Patch . . . . . . . . . . . . 20

2.2.2 Modelling the Contact Patch Curvature . . . . . . . . . . 24

2.2.3 The Tread Simulation Program . . . . . . . . . . . 25

2.3 Tire Empirical Model . . . . . . . . . . . . . . . . . . . . . . . 29

2.3.1 Lateral and Longitudinal Force Model . . . . . . . . . . . . 30

2.3.2 Aligning Torque Model . . . . . . . . . . . . . . . . . . 30

2.3.3 Turn Slip Model . . . . . . . . . . . . . . . . . . . . . . . 31

2.4 Tire Semi-Empirical Model . . . . . . . . . . . . . . . . . 34

2.4.1 The Similarity Method . . . . . . . . . . . . . . . . 34

2.5 Single Contact Point Transient Tire Models . . . . . . . . . . . . . . 37

2.5.1 Transient Deformation of the Contact Patch Centre . . . . . . 38

3 Tire Model Development $\quad 44$

3.1 Empirical Testing . . . . . . . . . . . . . . . . . . . . 44

3.1.1 Tire Test Facility Setup . . . . . . . . . . . . . 45

3.1.2 Instrumentation and Data Acquisition . . . . . . . . . 51

3.1.3 Tire Test Experiment and Results . . . . . . . . . . . . . . . . 54

3.1.4 Computational Model Validation and Results . . . . . . . . 59

3.1.5 The Magic Formula Tire Model . . . . . . . . . . . . . . . . . 62

3.1.6 The Similarity Method Tire Model . . . . . . . . . . . . . 70

4 Real Time Tire Computational Simulation $\quad 73$

4.1 The HeliMan Computational Simulation . . . . . . . . . . . . . . . . 73

4.1.1 Tire Models Comparison . . . . . . . . . . . . . 75

4.1.2 New Tire Model Application . . . . . . . . . . . . . 78

4.2 Validation of the Real Time Tire Simulation . . . . . . . . . . . 79 
Appendices

A Test Equipment and Calibration 


\section{List of Figures}

1.1 Different elements of the helicopter/ship dynamic interface. . . . . . . 2

1.2 Illustration of the tire string relaxation length. . . . . . . . . . . 7

2.1 Velocities associated with the contact patch centre. . . . . . . . . 14

2.2 Tire brush model demonstrating longitudinal slip. . . . . . . . . . 15

2.3 A typical longitudinal force versus $\kappa$ curve. . . . . . . . . . . . . . . . 16

2.4 Tire brush model demonstrating lateral slip. . . . . . . . . . . . 17

2.5 A typical lateral force versus $\alpha$ curve. . . . . . . . . . . . . 17

2.6 An illustration of contact patch rotation. . . . . . . . . . . 18

2.7 Tire contact patch. . . . . . . . . . . . . . . 20

2.8 Top view of a tire inclined by a camber angle. . . . . . . . . . . . . . 21

2.9 Reduction of radius along width of contact patch. . . . . . . . . . 22

2.10 Discritization of the tire contact patch. . . . . . . . . . 26

2.11 Tire tread deformation relative to the ground. . . . . . . . . . . . . 27

2.12 Graphical Solution to Equation 2.33 . . . . . . . . . . 29

2.13 Lateral force versus slip angle with turn slip. . . . . . . . . . . . 33

2.14 Illustration of the single contact patch tire model. . . . . . . . . . 38

2.15 Determining the lateral deflection from an $\alpha$ input. . . . . . . . . 41

2.16 Determining the lateral deflection from a $\gamma$ input. . . . . . . . . . . 42 


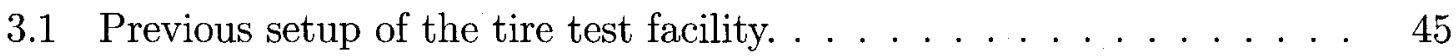

3.2 Oblique view of the tire test facility configured for aircraft tire testing. 46

3.3 Longitudinal view of the tire test facility configured for aircraft tire

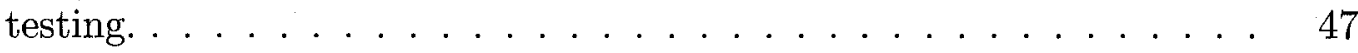

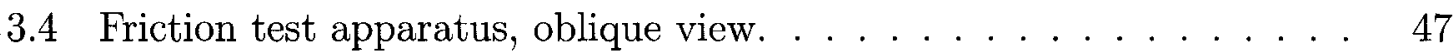

3.5 Friction test apparatus, side view. . . . . . . . . . . 48

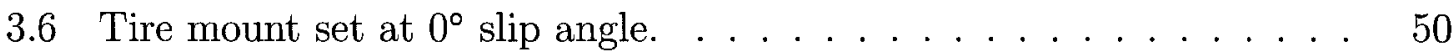

3.7 Tire mount set at $45^{\circ}$ slip angle. . . . . . . . . . . . . . . 50

3.8 X-Y load cell used to measure tire shear forces. . . . . . . . . . . 51

3.9 Axial load cell used to measure tire static normal force. . . . . . . . 52

3.10 Potentiometer used to measure carriage displacement. . . . . . . . . 52

$3.11 \mathrm{X}-\mathrm{Y}$ load cell mounted in the axial load testing machine. . . . . . . 53

3.12 A map of the steady state tire modelling. . . . . . . . . . . 55

3.13 Lateral force versus slip angle empirical test results with $351 \mathrm{lb}$ normal load on an Airtrac 6.00-6 8 ply tire for various camber angles. . . . . 56

3.14 Aligning torque versus slip angle empirical test results with $351 \mathrm{lb}$ normal load on an Airtrac 6.00-6 8 ply tire for various camber angles. $\quad 56$

3.15 Lateral force versus slip angle empirical test results with $509 \mathrm{lb}$ normal load on an Airtrac 6.00-6 8 ply tire for various camber angles. . . . . 57

3.16 Aligning torque versus slip angle empirical test results with $509 \mathrm{lb}$ normal load on an Airtrac 6.00-6 8 ply tire for various camber angles.

3.17 Lateral force versus slip angle empirical test results with $591 \mathrm{lb}$ normal load on an Airtrac 6.00-6 8 ply tire angles. . . . . . . . . . . . 58

3.18 Aligning torque versus slip angle empirical test results with $591 \mathrm{lb}$ normal load on an Airtrac $6.00-68$ ply tire angles. . . . . . . . . 58 
3.19 Comparison of the TreadSim computer model results to the empirical data for lateral force versus slip angle. . . . . . . . . . . . 60

3.20 Comparison of the TreadSim computer model results to the empirical

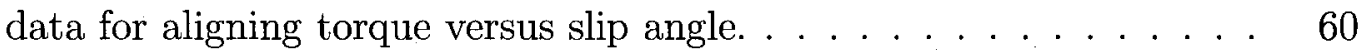

3.21 Lateral force versus slip angle with turn slip. . . . . . . . . . . 61

3.22 Aligning torque versus slip angle with turn slip. . . . . . . . . . 61

3.23 Magic Formula model fit of the lateral force versus slip angle to the empirical data. . . . . . . . . . . . . . . . 64

3.24 Magic Formula model fit of the aligning torque versus slip angle to the

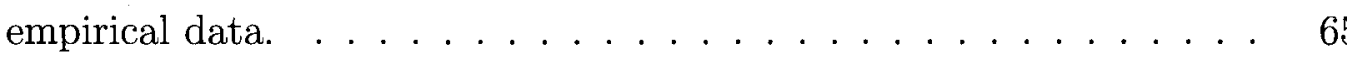

3.25 Magic Formula model fit of the aligning torque versus slip angle using

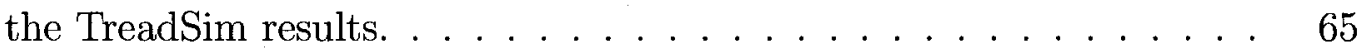

3.26 Magic Formula model fit of the $S_{H_{y \varphi}}$ spacing caused by turn slip. . . 67

3.27 Magic Formula model fit of $\zeta_{3}$ caused by turn slip. . . . . . . . . 67

3.28 Magic Formula fit of the lateral force versus slip angle with turn slip. $\quad 68$

3.29 Residual torque peak value versus turn slip. . . . . . . . . . . . 69

3.30 Aligning torque versus slip angle with turn slip. . . . . . . . . 69

3.31 Comparison of the lateral force versus slip angle curve generated by the Similarity Method to the empirical data collected with the normal

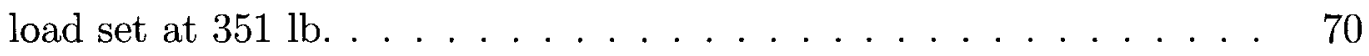

3.32 Comparison of the aligning torque versus slip angle curve generated by the Similarity Method to the empirical data collected with the normal

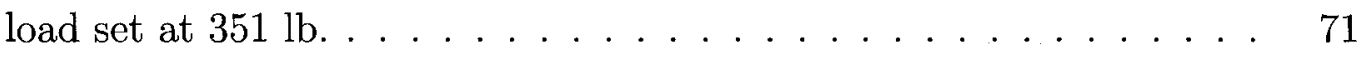

3.33 Comparison of the lateral force versus slip angle curve generated by the Similarity Method to the empirical data collected with the normal load set at $592 \mathrm{lb} \ldots \ldots \ldots \ldots \ldots \ldots$. . . . . . . . . . . . . . . . 
3.34 Comparison of the aligning torque versus slip angle curve generated by the Similarity Method to the empirical data collected with the normal

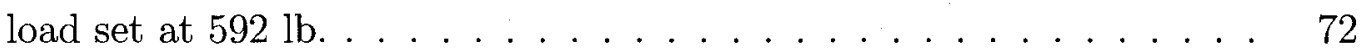

4.1 Schematic of the dynamic helicopter model. . . . . . . . . . . . 74

4.2 Free-body diagram of the main helicopter body and castorable wheel

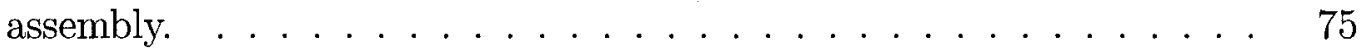

4.3 Block diagram of the computational tire model. . . . . . . . . . 78

4.4 Dead load test vehicle emulating a Seahawk helicopter. . . . . . . . 80

4.5 A representation of the computational simulation. . . . . . . . . 81

4.6 Comparison of the RSD claw y-position for Case 1. . . . . . . 82

4.7 Comparison of the force acting on the probe in the DLTV's y-direction for Case $1 \ldots \ldots \ldots \ldots \ldots$

4.8 Comparison of the force acting on the probe in the DLTV's x-direction for Case 1. . . . . . . . . . . . . . . . . . . . 83

4.9 Comparison of the lateral deflection of the right tire for Case 1. . . 84

4.10 Comparison of the lateral deflection of the left tire for Case 1. . . . 84

4.11 Initial position of DLTV and direction RSD is moved for Case 2. . . . 85

4.12 Comparison of the RSD claw y-position for Case $3 \ldots \ldots \ldots$

4.13 Comparison of the RSD claw x-position for Case $3 . \ldots \ldots$. . . . 87

4.14 Comparison of the castor orientation for Case $3 \ldots \ldots \ldots$. . . 87

4.15 Comparison of the helicopter orientation for Case $3 \ldots \ldots \ldots 8$

4.16 Comparison of the force acting on the probe in the DLTV's y-direction for Case $3 \ldots \ldots \ldots$

4.17 Comparison of the force acting on the probe in the DLTV's x-direction for Case $3 \ldots \ldots \ldots$. . . . . . . . . . . . . . . . . 89

xvii 
4.18 Comparison of the lateral deflection of the left tire for Case $3 . \ldots 90$

4.19 Comparison of the lateral deflection of the right for Case 3. . . . . . 90

4.20 Initial position of DLTV and direction RSD is moved for Case 4. . . . 91

4.21 Comparison of the RSD claw y-position for Case 4. . . . . . . . 91

4.22 Comparison of the RSD claw x-position for Case 4. . . . . . . . 92

4.23 Comparison of the castor orientation for Case $4 \ldots \ldots$. . . . . 92

4.24 Comparison of the helicopter orientation for Case $4 \ldots \ldots$. . . . 93

4.25 Comparison of the force acting on the probe in the DLTV's y-direction for Case $4 \ldots \ldots \ldots$. . . . . . . . . . . . . . . . . . . 93

4.26 Comparison of the force acting on the probe in the DLTV's x-direction

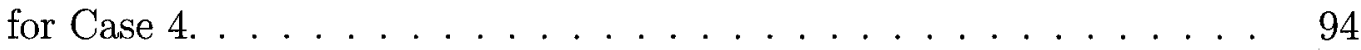

4.27 Comparison of the lateral deflection of the left tire for Case 4. . . . 94

4.28 Comparison of the lateral deflection of the right for Case 4 . . . . . 95

4.29 Comparison of the RSD claw y-position for Case $7 \ldots \ldots$. . . . . 97

4.30 Comparison of the RSD claw x-position for Case 7. . . . . . . . . 97

4.31 Comparison of the castor orientation for Case $7 \ldots \ldots \ldots$

4.32 Comparison of the helicopter orientation for Case $7 \ldots \ldots \ldots$

4.33 Comparison of the force acting on the probe in the DLTV's y-direction for Case $7 \ldots \ldots \ldots \ldots \ldots \ldots$

4.34 Comparison of the force acting on the probe in the DLTV's x-direction for Case $7 \ldots \ldots \ldots \ldots$

4.35 Comparison of the lateral deflection of the left tire for Case 7. . . . 100

4.36 Comparison of the lateral deflection of the right for Case $7 \ldots \ldots$. . 100

A.1 Calibration of load cell 7745 x-force. . . . . . . . . . . . . . 114

A.2 Calibration of load cell $7745 \mathrm{y}$-force. . . . . . . . . . . . 114

xviii 
A.3 Calibration of load cell $7700 \mathrm{x}$-force. . . . . . . . . . . . 115

A.4 Calibration of load cell $7700 \mathrm{y}$-force. . . . . . . . . . . . 115

xix 


\section{List of Tables}

3.1 Results of the non-skid deck friction tests . . . . . . . . . . . . . 49 


\section{Chapter 1}

\section{Introduction}

\subsection{Motivation}

The purpose of this thesis is to develop an improved tire model appropriate for use with a mathematical model and computer simulation developed for analyzing the motion and interface forces associated with a helicopter straightening and traversing on a ship flight deck. The real time helicopter traversing simulation named HeliMan was developed by Linn and Langlois [1]. The aircraft tires produce the reaction forces between the helicopter and the flight deck, and therefore play a pivotal role in the dynamic behaviour of the system.

Research aimed at improving the performance of helicopter operations on ships is of importance due to the advantages the helicopter brings to the ship's safety and effectiveness. A helicopter will improve a ship's response time and range of influence. Militaries use helicopters for security, search and rescue, and general utility. Helicopters can engage encounters that pose possible threats at safe distances from the ship in a swift manner. Search and rescue is improved by shipboard helicopters due to the increased range of the search area and in the reaction time of a rescue. 
Canada's military has been using helicopters onboard ships since the 1950's with sea trial experiments having begun in 1956.

The steps involved in the recovery of a helicopter onto a ship are outlined in Figure 1.1. They involve the helicopter's approach to the ship, the hovering and landing phase, the securing of the helicopter, the engage/disengage of the rotor, and the manoeuvring and traversing of the helicopter on the ship flight deck. Postural stability studies the ability of the crew to function without the need to actively balance to avoid a fall. All aspects of the helicopter recovery are subjects of ongoing research. A summery of the helicopter recovery research can be found in Linn [1].

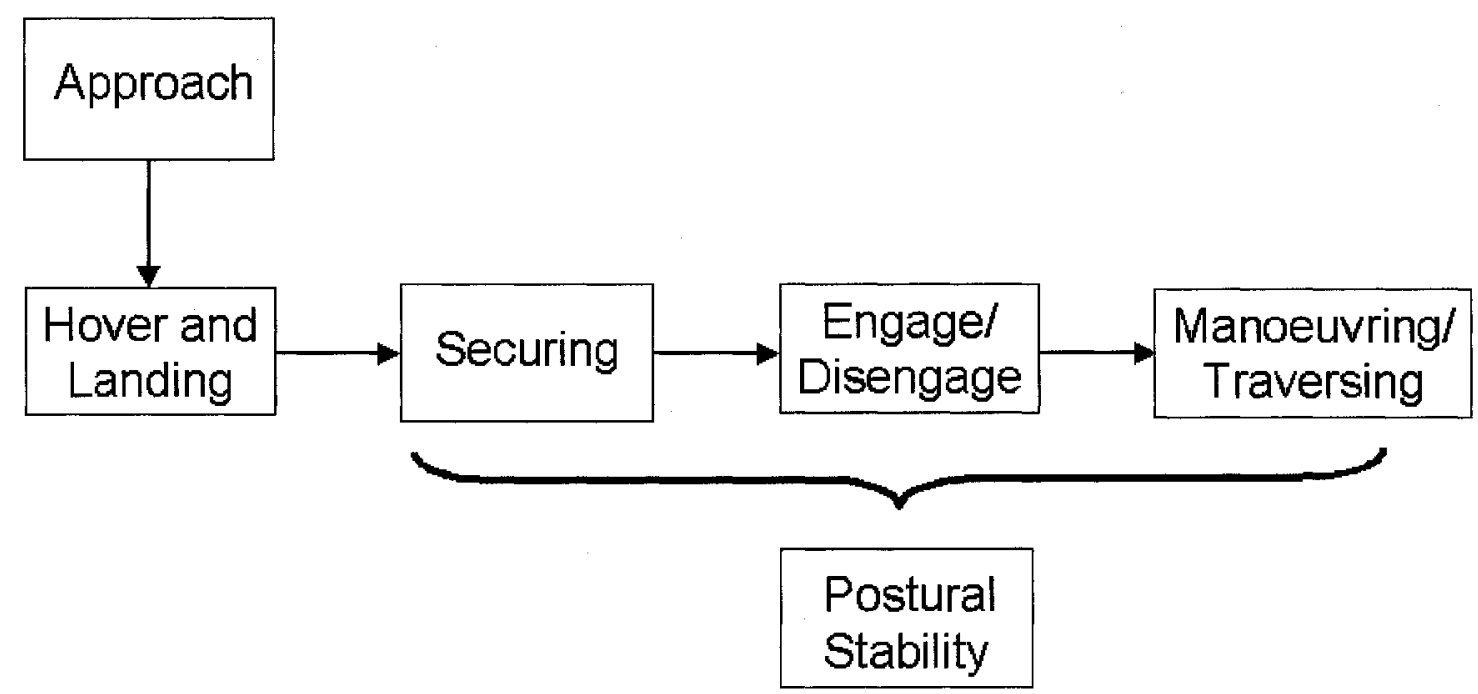

Figure 1.1: Different elements of the helicopter/ship dynamic interface.

In order to reduce the need for crew interaction with the helicopter recovery process, the recovery mechanisms are often designed for both capturing the helicopter upon touchdown as well as manoeuvreing and traversing the helicopter on the ship flight deck. The system that the HeliMan simulation models is that of the Aircraft Ship Integrated Secure and Traverse (ASIST) system. The ASIST system uses an electro-optical tracking system that strategically jockeys the Rapid Securing Device (RSD) position under the hovering helicopter. Light signals are also used to direct 
the pilot to the position of the RSD. The RSD autonomously traps the landing probe upon touch down. ASIST's RSD provides control in both the longitudinal and lateral direction.

The manoeuvring and traversing portion of the dynamic interface is the focus of this research. It is also the area that has least been explored academically. There are several reasons that motivate the development of a simulation for this phase of helicopter recovery. Simulations are useful in the development phase to predict the behaviour of the system. In the case of HeliMan developed by Linn and Langlois [1], the model can be used to further analyze the traversing system, offering potential improvements and predicting unusual operating conditions. The model can also be used for training an operator of the system. In the case of the ASIST system initial training currently occurs with actual hardware. Finally, the model can be used for development of automated control algorithms for the traversing system.

\subsection{Tire Simulation}

When simulating a naval helicopter traversing on a ship flight deck, the forces of interaction between the helicopter's tires and the ship's flight deck play a major role in determining the overall dynamic reaction of the system. Since the tire model plays a pivotal role in determining the dynamics of the system, it is important that an accurate mathematical representation be chosen to model the tire's behaviour. For the real-time helicopter simulation, the choice of a tire model must also allow for the tire's behaviour to be computed faster than real time. There are many choices of tire models ranging from complicated finite element models to pure empirical fitting models. The accuracy of the model often relies on the complexity of the mathematical formulation or from the number of experimental tests required to derive the model. 
Some of the popular tire models will be discussed below while pursuing the goal of choosing the best model for the purpose of the real-time helicopter simulation.

Many of the analytical tire models are derived by dividing all or part of the tire into discretized elements. Assumptions can then be made about the elements in order to derive their mechanical properties. Other models simplify the tire's geometry by representing it as a group of strings, beams, rings, or spokes. As a result, their mechanical properties can then be represented mathematically.

There are several finite element models in development that emulate the geometry of a complete tire including work by Hashimoto et al [2], Yu and Aboutorabi [3], Shiraishi et al [4], and Negrus et al [5]. The application of the finite element tire models ranges from steady state behaviour of the tire under conditions of contact patch slip, transient tire behaviour, to mode shape and eigenvalue analysis in the frequency domain. The finite element tire models produce results that correlate well with empirical test data. However, accuracy increases with the degree of complexity of the model. The complexity can range from a cylindrical tire with square and triangular elements to complicated models that use separate elements to simulate the ply and rubber individually as well as modelling complex tread geometry.

Similar to the complex finite element models are the tire models that simplify the geometry. Most of these techniques present surprisingly accurate results when compared with actual test data and have the advantage of shorter computation cycle time due to the simplicity of the tire model compared with finite element analysis models.

Perhaps the simplest tire model in this category is the tire brush model. The mathematics and results generated by this model are published by Pacejka [6]. The tire's contact patch is modelled by rectangular elements extending from one or more longitudinal spines representing the rigid portion of the tire to the contact point with 
the ground. Figures 2.2 and 2.4 in Chapter 2 illustrates side and top views of this model. As the tire moves along the ground the rectangular elements will adhere to the ground and experience slip under the right cornering and driving torque conditions. Therefore, the top of the rectangular element will be moving with the rigid portion of the tire while the ground contact point either adheres to the ground or skids across the ground when the deformation force exceeds the frictional force. The difference in position and velocity of the top portion of the rectangular element relative to the bottom portion of the element represents the tire deformation and the rate of deformation respectively. The tire brush model is not used to model actual tire behaviour. The rigid nature of the tire carcass exaggerates the magnitudes of the tire behaviour. The trends of the behaviour accurately model the expected trends of the tire observed in reality, allowing the model to serve as an observational tool to better understand models of greater complexity.

The stretched string based tire model is a technique widely used to model the tire's contact patch behaviour with greater accuracy when compared to that of the brush model and to those consisting of beams. The model consists of one or more parallel longitudinal strings of infinite length. A portion of the string will be in contact with the ground. The remainder of the string represents the circular portion of the tire not in contact with the ground. The string may deform in the axial direction of the tire, while the deformation is restricted in the circumferential direction. The deformable carcass of the tire and the tire ground interaction present the boundary conditions for the strings. The mathematics, developed by Pacejka [6], associated with this model are presented in Chapter 2. Essentially, the model's mechanics is similar to that of the brush model in the sense that the model compares a point on the contact patch to its corresponding point on the deformable tire carcass located next to the hub of the tire. As adhesion and skidding changes the velocity of the contact patch relative 
to the carcass, deformation occurs creating the corresponding tire force. This process is then considered for every point on the contact patch resulting in the total contact patch deformation and force moment generation. Modelling the carcass deformation accurately results in a tire model that correlates well with actual empirical results.

The stretched string tire models have been used to study tire behaviour for steady state analysis, transient analysis, and frequency domain analysis. Zegelaar et al [7] use the string model to determine mode shapes and frequency response of a tire. Nybakken et al [8] demonstrate the effectiveness of the string model by comparing the model results to tire test results. Mastinu et al [9] use a technique similar to the string model for modelling the contact patch deformation. However, the contact patch is broken into elements and the boundary conditions for the side walls are set up using tire inflation pressure.

The spoke tire model consists of a circular hub with poles extending from the hub outwards in the circumferential direction of the tire. The spokes deform in their longitudinal directions resulting in the overall shape of the tire. This model was used to study the frequency response of a tire by Wong and Sharp [10].

A popular transient tire model that incorporates the stretched string theory is the single contact point transient tire model. The mathematical behaviour of the model is controlled by a parameter called the tire relaxation length. The leading edge relaxation length is the distance between the point on the string that is the leading edge of the tire contact patch and the point where the string is no longer laterally deformed, as shown in Figure 1.2. The trailing edge can also be given a relaxation length. In the case of the single contact point tire model, the contact patch centre is the only point modelled and lateral deformation will lead to a single relaxation length. The derivation of this model is reported by Pacejka [6] and by Higuchi and Pacejka [9]. The set of equations derived by modelling the tire behaviour from a 


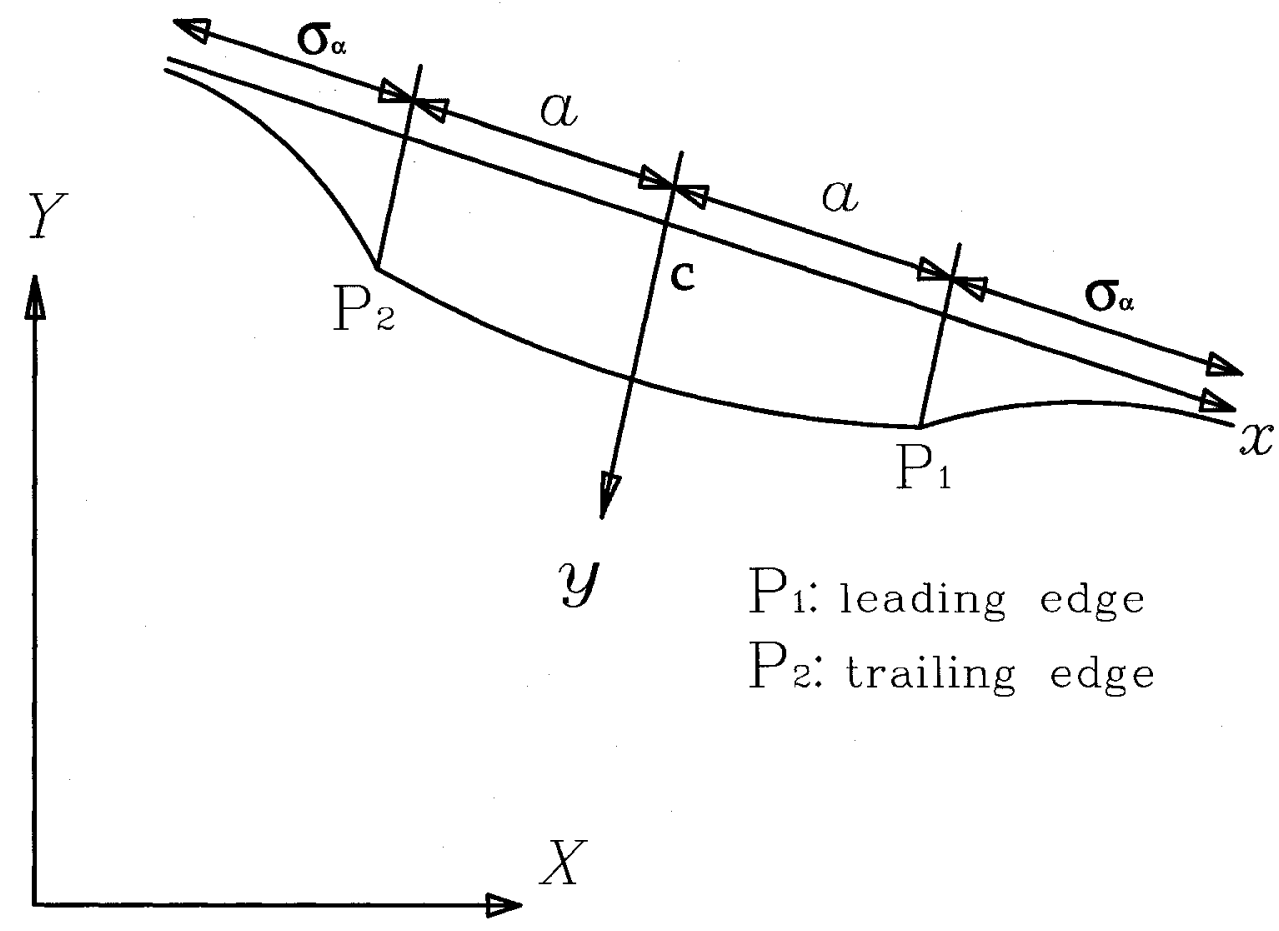

Figure 1.2: Illustration of the tire string relaxation length.

single contact point are nonlinear and can be solved by determining the relaxation length of the tire with the help of a steady state tire model. The equations can also be linearized and used for the same input values. The linearized form of the equations are often good enough for vehicle simulation.

Empirical tire models, through the use of mathematical equations, simulate the trends produced from experimental testing. They range in complexity, generally requiring a greater number of experiments for a more accurate tire model. One such model is the Magic Formula tire model presented by Pacejka and Besselink [11]. The Magic Formula Tire Model is a family of curves that fit the results of steady state tire experiments with great accuracy. The model can simulate the effects of force and moments generated by inputs of lateral, longitudinal, and turn slip as well as from changes in camber angle and tire normal force. A full development of the 
Magic Formula tire model is also presented by Pacejka [6], including the modelling of turn slip. Several implementations that have incorporated the Magic Formula tire model include those of Hoffmann et al [12] that incorporate the Magic Formula model into their vehicle simulation, Tezuka et al [13] that use the model in a motorcycle simulation with emphasis on the camber angle input, and Slagmaat [14] where the Magic Formula model is used in aircraft landing gear simulation.

One disadvantage with the pure Magic Formula tire model is the large number of tire experiments required in order to use the model with normal force as a variable input. Pacejka [6] observed that the shapes of the pure longitudinal, lateral, and aligning torque curves remained the same under different operating points of normal force or surface friction. It was observed that a non-dimensional curve could be created by normalizing the variables. This allowed for the development of a model that adjusted the tire behaviour from a group of reference variables when a different normal force or surface friction coefficient was used. The model's name is the Similarity Method, and the model can adjust for the steady state tire characteristics under new normal force loading. Fewer tests are required on the tire at different normal forces to develop the Similarity Method tire model than what is required by the pure Magic Formula model. Test procedures were developed by Oosten and Bakker [15] and by Leister [16] to determine the parameters required by the Magic Formula tire model and the Similarity Method tire model.

Tire modelling in the aviation industry has largely focused on an empirical tire study conducted by Smiley and Horne [17]. The report created an empirical model from test results conducted on modern aircraft tires of the time, originally published by NACA in 1958 and later re-published by NASA in 1960. The model determines the vertical deflection of the tire based on the amount of normal force and tire pressure. It then determines the amount of tire lateral and longitudinal deformation and 
reaction force. The deformation and reaction force is analogous to the single contact transient model discussed above. The model uses the steady state force and the relaxation length from empirical test data. Investigation of the transient portion of the model, reveals that the relaxation length and steady state force is only adjusted to the variable of normal force and not from slip which is required for a complete nonlinear version of the single contact point model.

Many aviation simulation projects have incorporated the Smiley and Horne model including the original version of HeliMan produced by Linn and Langlois [1]. Reference to the use of the Smiley and Horne model have also been made in $[18,19,20,21]$ that focused on aircraft landing and taxiing.

Daugherty [22] conducted tire experiments at the Langley Research Center using three large radial ply commercial tires. The results show a large difference in tire behaviour between the radial ply tires and the bias ply tires which were used by Smiley and Horne. This implies that the empirical model of Smiley and Horne might be obsolete for modern aircraft tires.

The literature review lead to the following tire model development. The new tire model will be developed by modelling the steady state tire behaviour with the Magic Formula method and Similarity Method through empirical testing. The transient simulation will then incorporate the steady state model to solve the fully nonlinear single contact point model. This model will allow for large slip angles to occur and allow for comprehensive modelling of turn slip and camber.

\subsection{Objectives}

The objective of this thesis is to improve the existing HeliMan simulation with a tire model that handles large input quantities of lateral slip for a slow moving tire. The 
tire model must also handle continuous changes in camber, longitudinal and turn slip, and normal force.

In order to meet these objectives, tire tests will be conducted on an aircraft tire to develop the steady state tire model using Pacejka's Magic Formula model and Similarity Method model. In order to model turn slip, a string based contact patch simulation called TreadSim will be developed based on the empirical test results. Turn slip is an input that occurs during tire rotation about the contact patch centre. The effects of turn slip are generally small and negligible so long as the radius of a turn is large. In the case of castorable helicopter tail wheels (such as the widely used Sea King and Seahawk aircraft) the turn slip is required. The steady state model will then be used to solve the full nonlinear single contact patch transient tire model.

The model will be implemented into the HeliMan computational program and the results will be compared against full-scale tests previously conducted using a dead load test vehicle performed at Indal Technologies Inc. (ITI) and against the old tire model.

\subsection{Thesis Overview}

The thesis develops an improved tire model for the HeliMan simulation used to analyze helicopter manoeuvring and traversing on a ship flight deck under the influence of the ASIST handling system. This chapter motivates the research and presents several of the current tire models in use and under development. Chapter 2 presents the mathematical theory behind the tire models used in the simulation development. Chapter 3 presents the developed tire model and compares the results with empirical test results obtained for an aircraft tire. Chapter 4 applies the tire model to the HeliMan simulation and compares the results to the empirical test data and the 
previously implemented Smiley and Horne [17] model. Chapter 5 provides discussion and presents the conclusions drawn from this study. 


\section{Chapter 2}

\section{Tire Model Theory}

\subsection{Tire Input Quantities}

The forces acting on a moving tire vary depending on a number of variables that include normal force, pressure, tire inclination, and contact patch slipping. The variables that vary the most during tire traversing are due to tire slipping, which is a result of slip angle, longitudinal slip, and turn slip. These slip quantities are ideal input parameters for tire force analysis and will be explained subsequently.

The simplest dynamic case of a tire in motion is that of an upright free rolling tire. Its forward velocity tangent to its longitudinal axis $V_{r}$ is represented by

$$
V_{r}=r_{e} \Omega
$$

where $r_{e}$ is the tire's effective rolling radius and $\Omega$ is the angular velocity of the tire about its axis of rotation perpendicular to the plane containing the tire. There is a need to further define the tire's radius because the circumference of the tire changes due to normal force and longitudinal deformation that occurs as the tire slips. The "effective radius" is the value of the tire radius that will cause Equation 2.1 to hold 
true. The effective radius will be slightly less than the radius of an undeformed tire. A tire's velocity will differ from Equation 2.1 if the tire is slipping. $\vec{V}_{c}$ will represent the velocity of the centre of the contact patch. In typical vehicle models, the velocity of the wheel hub is the likely known velocity. The velocity of the hub and the velocity of the wheel contact patch centre will be equal in most cases unless a rotation exists between the two points. Rotations between the hub and the contact patch centre typically results from a change in terrain elevation and the differences that occur are often negligible. If a tire operating at steady state is on a flat road with rate induced affects neglected then the velocity of the contact patch centre can be represented by

$$
\begin{gathered}
V_{c x}=V_{r}-V_{s x} \\
V_{c y}=V_{s y}
\end{gathered}
$$

where $V_{s x}$ and $V_{s y}$ are the orthogonal components of $\vec{V}_{s}$ which represents the slipping speed of the contact patch centre. The sliding of the contact patch is due to deformation caused by adhesion to the contact surface and from sliding when the shear force exceeds the surface frictional force. For a freely rolling tire, point $\mathrm{c}$ is an instantaneous centre between the tire and the reference frame of the ground located at the centre of contact. If the tire is slipping then the motion is due to both free rolling and translation from deformation. The velocity at witch the "instantaneous centre" would have to travel in order to represent the free rolling is the slip velocity $\vec{V}_{s}$. Figure 2.1 illustrates the velocities associated with point $\mathrm{c}$.

An observer travelling at a rate equal to the free rolling velocity, will directly observe the slip velocities at the contact centre as well as any rotation occurring on the contact patch. Contact patch angular velocity, $\omega_{z}$, will add a new component of slip velocity to all the contact points surrounding the contact patch centre. Therefore, 


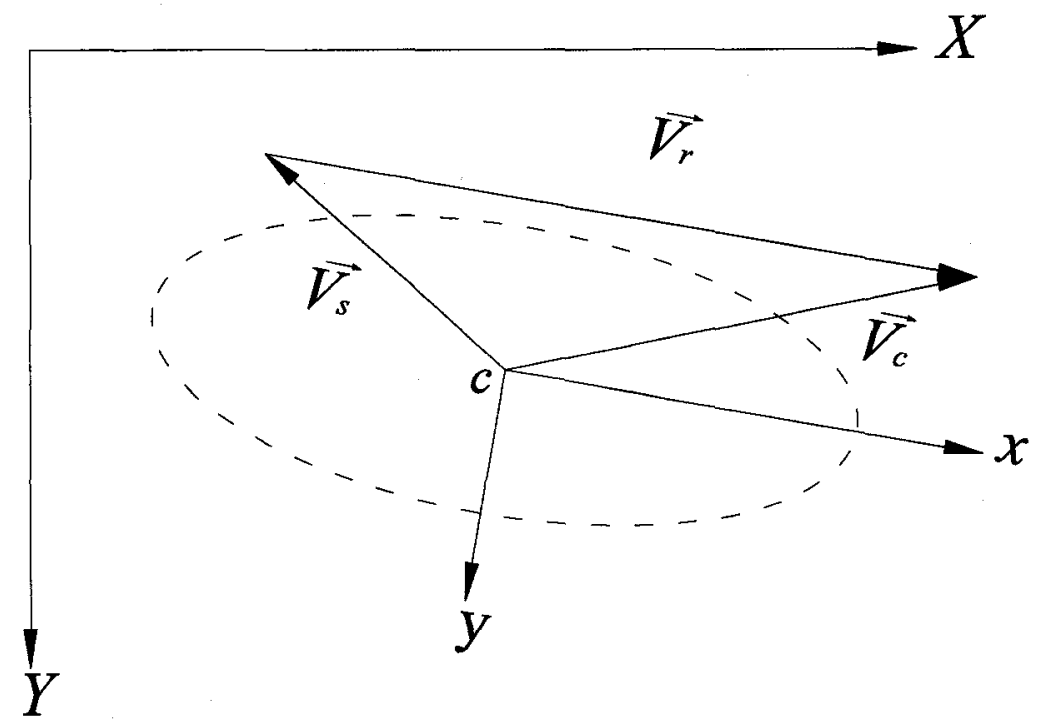

Figure 2.1: Velocities associated with the contact patch centre.

the forces that act on a tire can be categorized into inputs that cause translations about the tire's longitudinal and lateral axes, and those resulting from rotations acting about the tire's contact patch centre.

The first category of input slips are caused by translations of the contact patch. The two slip quantities used to measure the translational slips are known as the longitudinal slip, $\kappa$, and slip angle, $\alpha$. The two quantities are represented by

$$
\kappa=-\frac{V_{s x}}{V_{c x}}
$$

and

$$
\tan \alpha=-\frac{V_{s y}}{V_{c x}}
$$

The extremes of Equation 2.4 occur during wheel lock, which is the case of the tire undergoing pure translation, and wheel spin, the case when the tire is rotating with zero translational velocity. The value of $\kappa$ will be equal to -1 during wheel lock and 


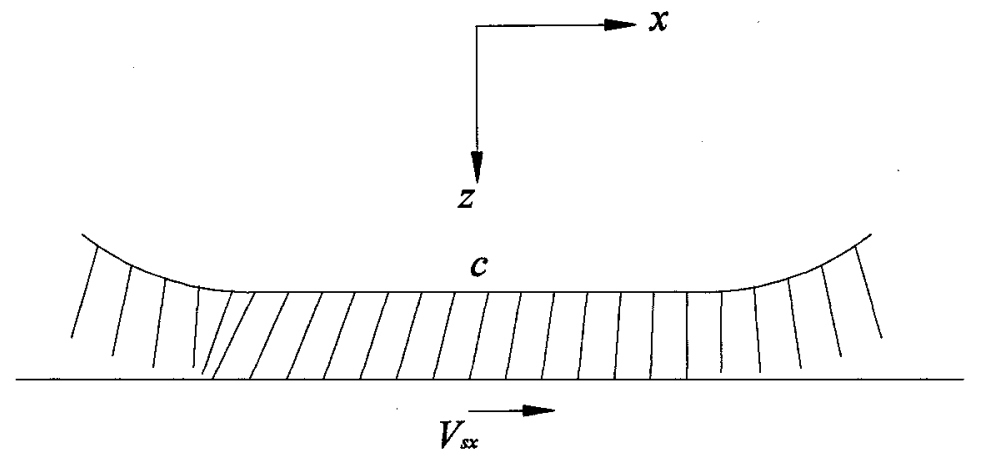

Figure 2.2: Tire brush model demonstrating longitudinal slip.

$\infty$ during wheel spin. A known quantity of torque or braking will correspond to an equal amount of longitudinal slip.

A visualization technique known as the tire brush model can be used to explain the deformation of the contact patch. Figure 2.2 shows a side view of the tire tread in contact with the ground and experiencing longitudinal braking slip (sometimes referred to as skid). If a point is observed on the circumference of a tire first coming into contact with the road, adhesion will first occur. Consequently, as the tire slips backwards (in the case of a driving torque being applied) the tread will deform. The further the observed point travels from the leading edge, the greater the deformation becomes. The deformed rubber will have an equal and opposite reaction force to that of road friction. When the maximum frictional force is reached, that equalling the product of normal force and the frictional coefficient, sliding will occur. With enough slip, the entire contact patch will eventually be in a state of sliding. The saturation of sliding on the contact patch is what gives rise to the familiar shape of the longitudinal force versus longitudinal slip curve, as illustrated in Figure 2.3. 


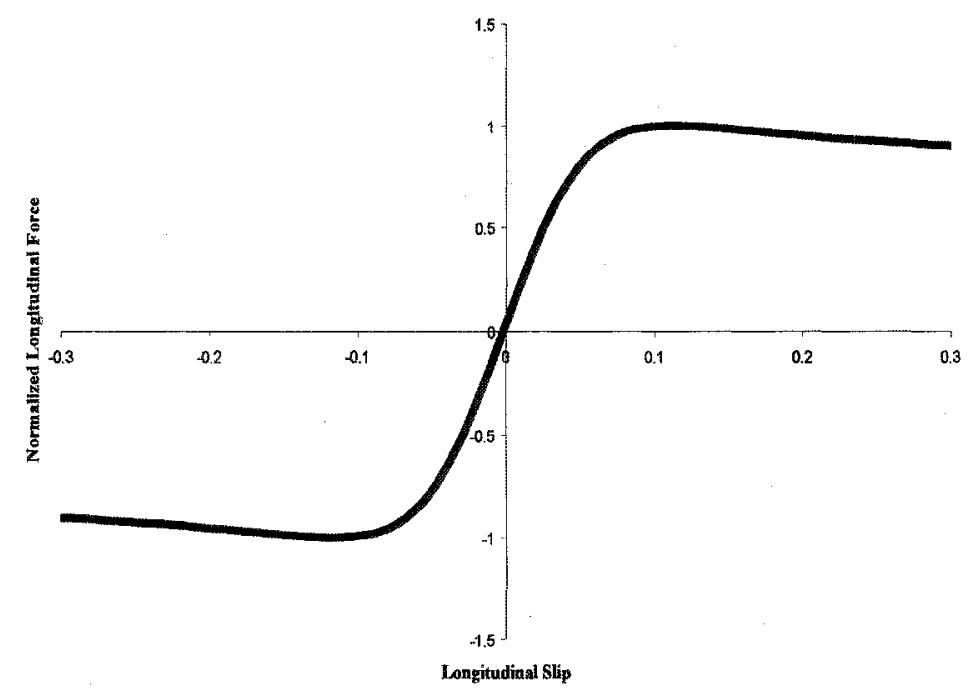

Figure 2.3: A typical longitudinal force versus $\kappa$ curve.

The brush model also illustrates the effect of lateral slip effectively. Figure 2.4 shows a top view of a longitudinal strip of contact patch. As the contact patch deforms, the "bristles" of the brush become visible. Similar to the case of longitudinal slip, as a point touches the ground at the leading edge of the contact patch, adhesion will occur. A point further back from the leading edge will have deformed proportionally to $-\frac{V_{s y}}{V_{c x}}$, until sliding occurs. The concentration of normal loading is assumed to have a parabolic distribution. The parabola in Figure 2.4 represents the maximum deformation that occurs before sliding. As the slip angle is increased, a larger portion of the contact patch will begin slipping until the entire contact patch is sliding. This causes a similar shaped curve compared to that of the longitudinal force versus $\kappa$ and is illustrated as Figure 2.5.

The slip caused by contact rotation is far more complicated than the previously described translational slips. There are two main factors that cause a tire to rotate about its $\mathrm{z}$ axis. One is caused from the tire travelling on a curved path, and the other is from a component of the tire's angular rotation when the tire is inclined away 


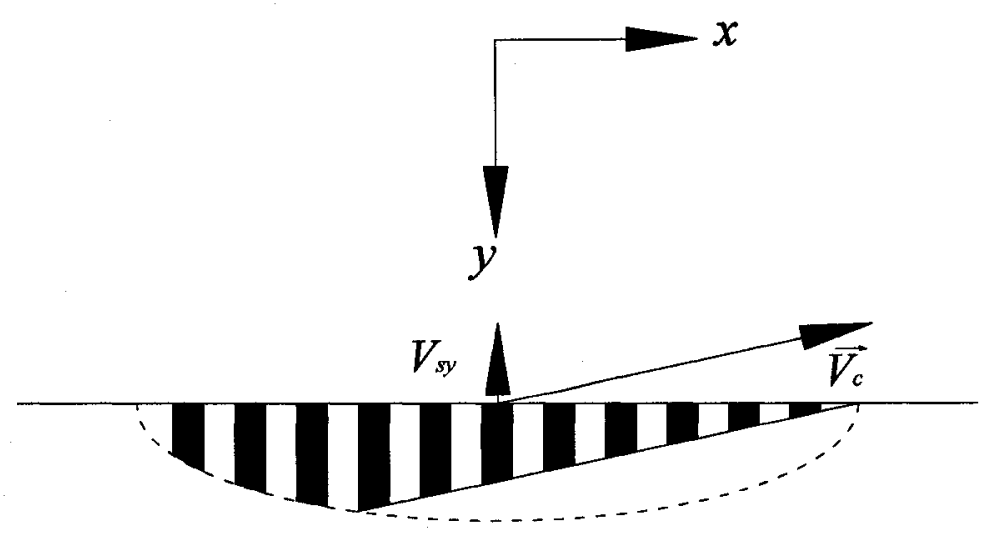

Figure 2.4: Tire brush model demonstrating lateral slip.

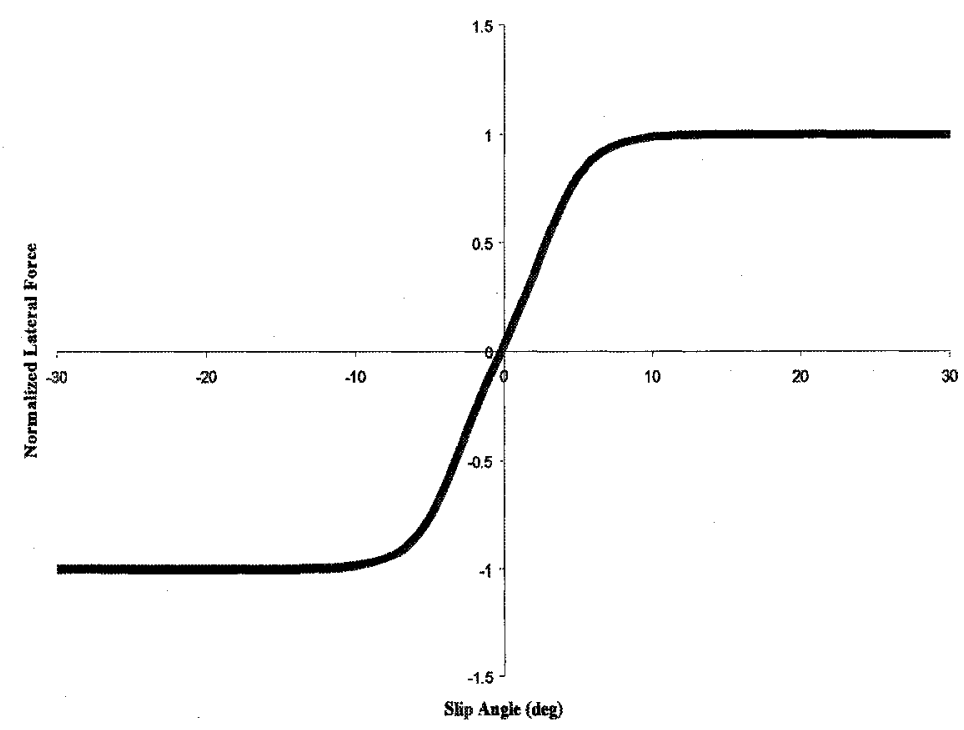

Figure 2.5: A typical lateral force versus $\alpha$ curve. 

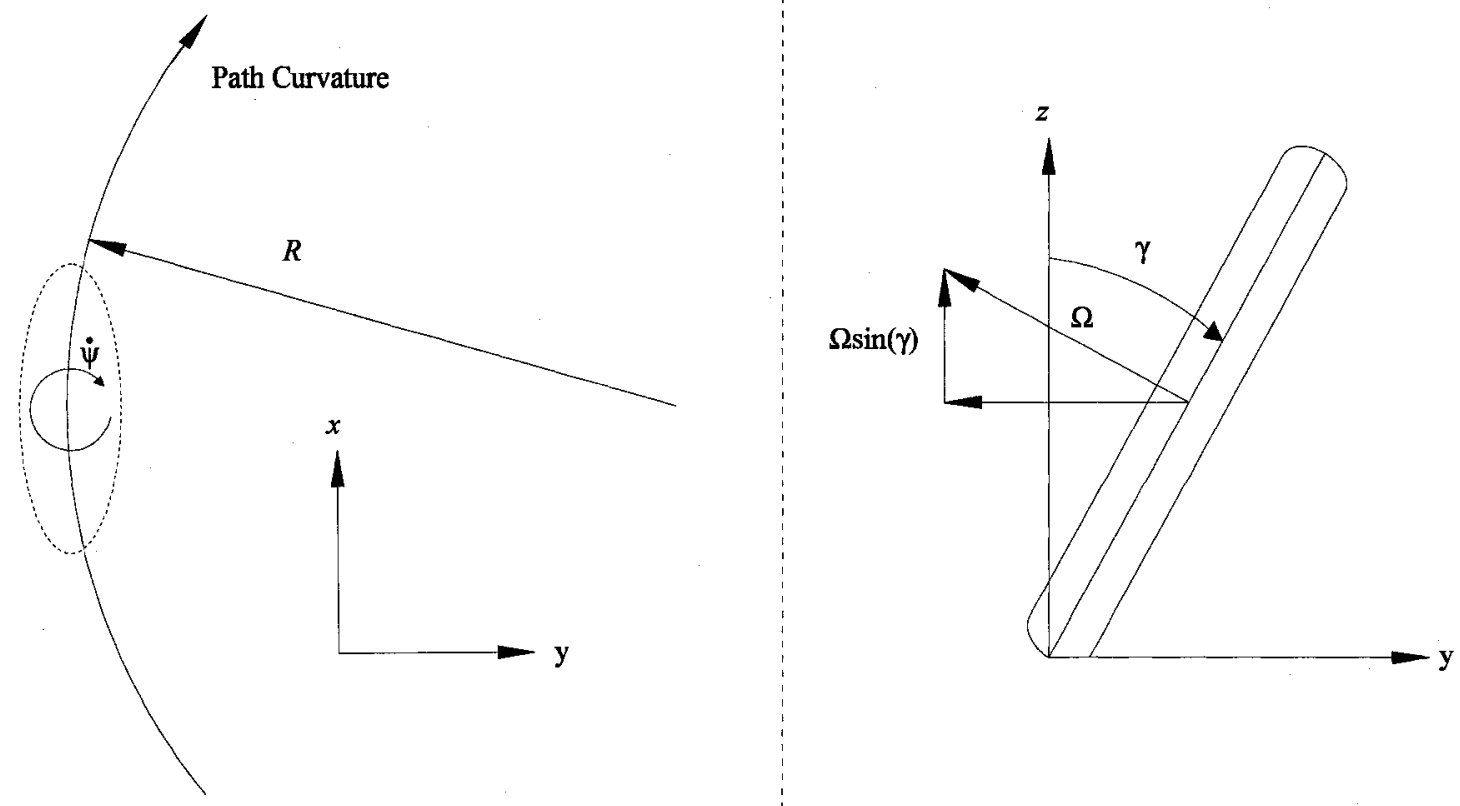

Figure 2.6: An illustration of contact patch rotation.

from an upright vertical position. The two cases are illustrated in Figure 2.6. The equation that represents $\omega_{z}$ is

$$
\omega_{z}=\dot{\psi}-\Omega \sin \gamma
$$

where $\dot{\psi}$ represents the angular velocity of the tire due to path curvature and $\gamma$ represents the camber angle defined as the tire inclination relative to the vertical plane. In most tire models the effect due to contact patch rotation is separated into the effects due to road curvature and the effects due to camber. Camber causes the lateral force versus slip angle curve to shift and stretches the aligning moment versus slip angle curve. The shifting and stretching that occurs to the force and 
moment curve are linearly proportional to the amount of inclination for small values of camber that are most often experienced. The changes due to path curvature are often negligible and disregarded in many tire models. However, if the radius of the path curvature becomes small, the slip generated from the path curvature will become sufficient such that its effects should not be ignored. Rotational slip is treated in one of two ways. Spin slip equals $-\frac{\omega_{z}}{V_{c x}}$. However, this is not often used; rather it is more common to treat curvature rotation and camber separately. If this is the case, then

$$
\varphi_{t}=\frac{-\dot{\psi}}{V_{c x}}
$$

which represents turn slip, is used.

\subsection{Computational Modelling}

Tread Simulation is a program developed by Pacejka [6] that determines the steady state forces acting on a tire by modelling the deformation of the tire's contact patch. The amount of deformation of any point on the contact patch is determined by the velocity and slip inputs applicable at that point. The program allows for modelling of tire behaviour for situations that are not easily testable in a controlled environment. Such situations include combined longitudinal and lateral slip inputs, large degrees of camber inclination, as well as modelling all tire behaviours with the influence of turn slip. The parameters for the tire model are chosen based on curve fitting the model's behaviour with the empirical data obtained from tire testing. Once the tire parameters are chosen, the model is then used to observe the tire behaviour as influenced by turn slip. 


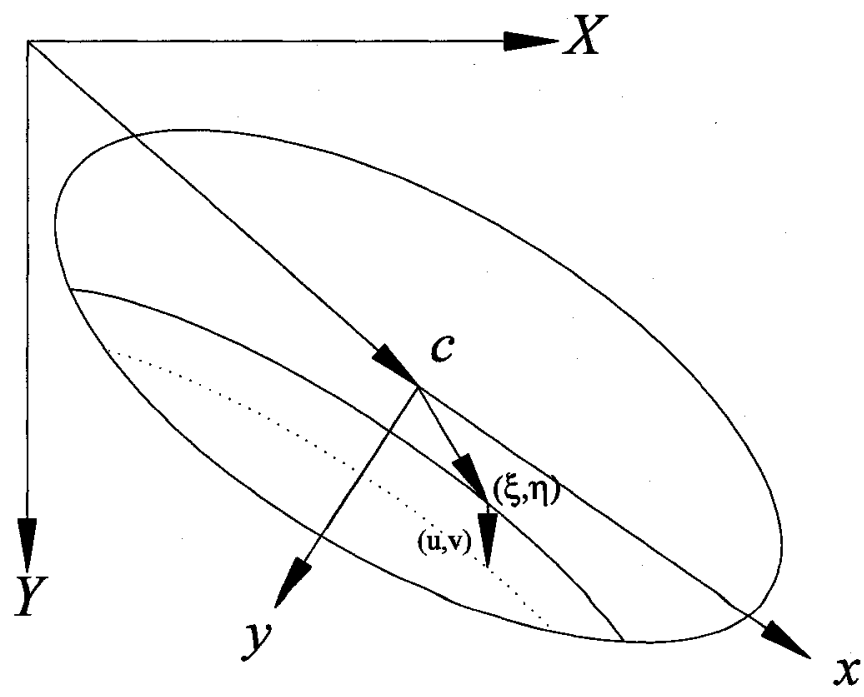

Figure 2.7: Tire contact patch.

\subsubsection{Motion of the Tire Contact Patch}

In order to model the motion of the tire's contact patch, several reference planes must be introduced. The stationary $(\mathrm{X}, \mathrm{O}, \mathrm{Y})$ reference plane will represent the ground that the tire traverses. The $(\mathrm{x}, \mathrm{c}, \mathrm{y})$ reference plain will move with the tire's contact patch centre point, c, and represents the tire's longitudinal $(\mathrm{x})$ and lateral $(\mathrm{y})$ axes. Both reference plains serve as orthogonal coordinate systems with their third coordinate vector, $\mathrm{z}$, pointing towards the ground. Top view of the tire's contact patch and the two coordinate systems are illustrated in Figure 2.7.

When a tire is inclined with a camber angle, the tire will prefer to follow a curved path. The curved path can be defined by viewing the cambered tire from a top view as in Figure 2.8. In this view the tire is not loaded with a normal force and the surface is considered frictionless. Three lines are drawn in Figure 2.8 representing the tire's circumference. The projection of these lines onto the ground plain represents the curvature path that the tire will try to follow.

When a normal force is added, the tire's rubber will deform due to compression 


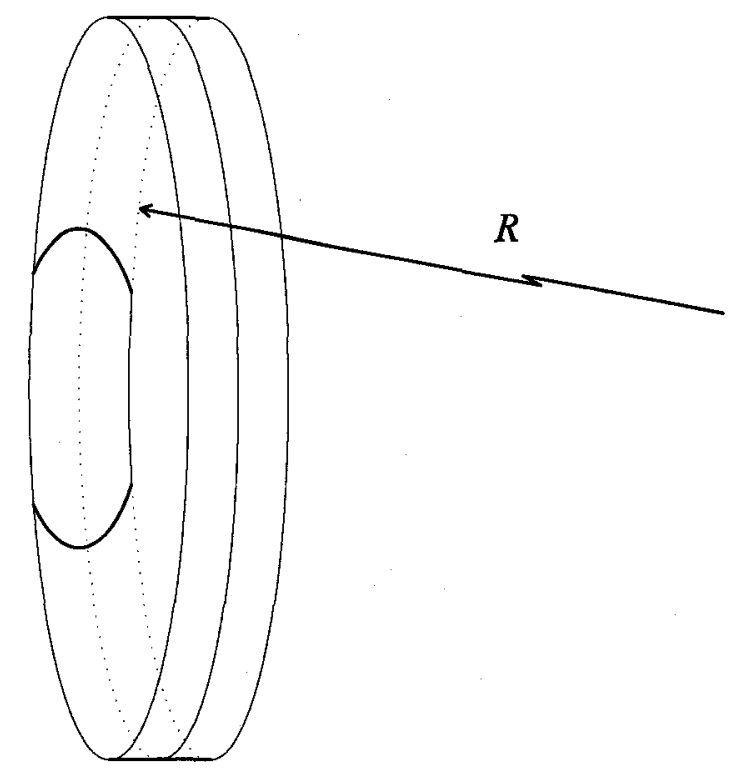

Figure 2.8: Top view of a tire inclined by a camber angle.

and the tire's carcass and belt will distort due to ply steer, conicity, and camber. A new set of coordinates $(\xi, \eta)$ represent the position of a point on the contact patch after the tire has been initially cambered and normally loaded. The velocity of the point $(\xi, \eta)$ travelling on a frictionless surface is represented by

$$
\begin{gathered}
\dot{\xi}=\dot{x}+\dot{u}_{o}(x) \\
\frac{\partial \xi}{\partial t}=\frac{d x}{d t}+\frac{\partial u_{o}}{\partial x} \frac{d x}{d t} \\
\frac{\partial \xi}{\partial t}=r_{o} \Omega\left[1+\frac{\partial u_{o}}{\partial x}\right] \\
\dot{\eta}=\dot{y}+\dot{v}_{o}(x) \\
\frac{\partial \eta}{\partial t}=\frac{d y}{d t}+\frac{\partial v_{o}}{\partial x} \frac{d y}{d t} \\
\frac{\partial \eta}{\partial t}=-\Omega\left[\xi \sin \gamma+\frac{\partial v_{o}}{\partial x} r_{o}\right]
\end{gathered}
$$

where $(\mathrm{x}, \mathrm{y})$ represents the coordinates of the contact patch point before normal load- 


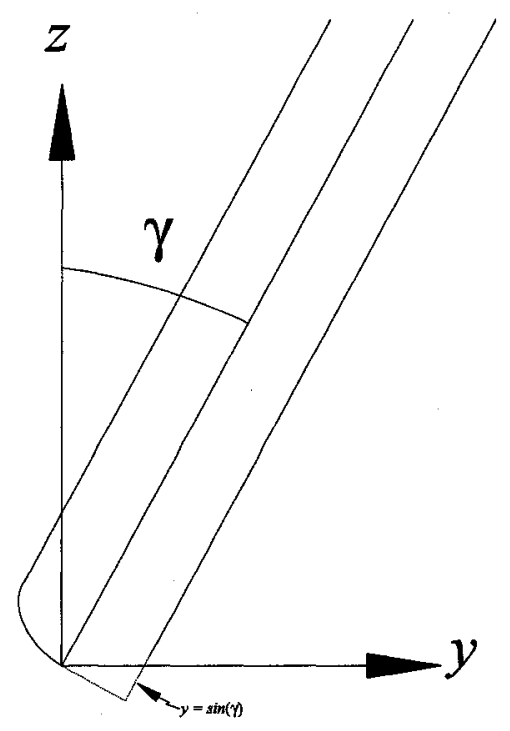

Figure 2.9: Reduction of radius along width of contact patch.

ing is applied and $\left(u_{o}, v_{o}\right)$ represents the initial deformation of that point with the addition of a normal load. The $\frac{\partial u_{0}}{\partial x}$ and $\frac{\partial v_{0}}{\partial x}$ terms represent the curvature change on the contact patch caused by camber and conicity after the tire has been normally loaded. The change in rolling radius along the width of the contact patch must also be considered. A tire that is inclined with a camber angle will experience compression of the tire's radius on the inside of the induced curvature path and an expansion on the outside of the curvature path. The radius change across the contact patch is illustrated in Figure 2.9.

To account for the radius change, Equations 2.8 and 2.9 are transformed to

$$
\begin{gathered}
\dot{\xi}=-\left[r_{e}+y \sin (\gamma)\right] \Omega-\left[r_{e}+y \sin (\gamma)\right] \frac{\partial u_{o}}{\partial x} \\
\dot{\xi}=-\left(1+{\frac{\partial u_{o}}{\partial x}}_{\operatorname{con} x(y)}\right) r_{e} \Omega-\left(1+{\frac{\partial u_{o}}{\partial x}}_{\gamma x(y)}\right) \eta \sin (\gamma) \Omega \\
\dot{\eta}=-\Omega\left[\xi \sin \gamma+\frac{\partial v_{o}}{\partial x} r_{e}\right]
\end{gathered}
$$


where $r_{e}$, the effective rolling radius, is the radius of the tire at the contact patch centre. In Equation 2.10, $\frac{\partial u_{o}}{\partial x}$ has been split into two quantities. One representing the change of curvature due to camber and the other a change in curvature due to tire conicity.

The sliding velocity of any point on the tire's contact patch relative to the ground can be represented by

$$
\vec{P}=\vec{V}_{c}+\vec{Q}
$$

where $\vec{V}_{c}$ represents the velocity of the contact patch centre in the upright position relative to the ground and $\vec{Q}$ represents the velocity of a point on the contact patch relative to the contact patch centre.

When a tire moves along a surface, its contact patch will experience deformation due to friction. The deformation of a point on the contact patch after an initial normal force and camber inclination have been applied will be represented by the variables $u$ and $v$ for deformation in the $(\mathrm{x}, \mathrm{y})$ directions. With vectors $\hat{i}, \hat{j}, \hat{k}$ representing the directions in the $(\mathrm{x}, \mathrm{y}, \mathrm{z})$ coordinate system, the rate of change of $\vec{Q}$ can be represented as

$$
\dot{\vec{Q}}=(\dot{\xi}+\dot{u}) \hat{i}+(\dot{\eta}+\dot{v}) \hat{j}+\dot{\psi}[(\xi+u) \hat{j}-(\eta+v) \hat{i}]
$$

The velocity of the contact patch centre can be represented as the velocity of the tire when free rolling $\vec{V}_{r}$ plus the slip velocity. The slip velocity, $\vec{V}_{s}$, is the rate of deformation at the contact centre. Therefore

$$
\begin{gathered}
\vec{V}_{r}=r_{e} \Omega \\
\vec{V}_{c}=\vec{V}_{r}+\vec{V}_{s}
\end{gathered}
$$

The deformation of a point on the contact patch depends on the location of the 
point and on time. Therefore

$$
\dot{u}=\frac{\partial u}{\partial \xi} \frac{d \xi}{d t}+\frac{\partial u}{\partial \eta} \frac{d \eta}{d t}+\frac{\partial u}{\partial t}
$$

and

$$
\dot{v}=\frac{\partial v}{\partial \xi} \frac{d \xi}{d t}+\frac{\partial v}{\partial \eta} \frac{d \eta}{d t}+\frac{\partial v}{\partial t}
$$

By neglecting small terms and considering the case where there is no lateral rolling Equation 2.12 becomes:

$$
\begin{gathered}
\dot{P} x=\vec{V}_{s} \cdot \hat{i}-\left(\frac{\partial u}{\partial x}+{\frac{\partial u_{o}}{\partial x}}_{\operatorname{con} x(y)}\right) V r+\frac{\partial u}{\partial t}-\eta(\dot{\psi}-\Omega \sin \gamma)+{\frac{\partial u_{o}}{\partial x}}_{\gamma x(y)} \eta \sin (\gamma) \Omega \\
\dot{P} y=\vec{V}_{y} \cdot \hat{j}-\left(\frac{\partial v}{\partial x}+\frac{\partial v_{o}}{\partial x}\right) V_{r}+\frac{\partial v}{\partial t}+\xi(\dot{\psi}-\Omega \sin \gamma)
\end{gathered}
$$

\subsubsection{Modelling the Contact Patch Curvature}

In order to use Equations 2.17 and 2.18 to represent a specific tire, the curvature characteristics $(\xi, \eta)$ must be properly defined. The equations that represent the curvature and curvature slope of the contact patch in terms of camber, conicity, plysteer, and external forces are given by:

$$
\begin{gathered}
\eta=-\frac{a^{2}}{2 r_{e}}\left(1-\frac{\partial v_{o}}{\partial x}\right)\left(\gamma_{c o n}+\sin (\gamma)\right)+\frac{F y}{C_{l a t}}+C_{s} \xi+\frac{1}{2} C_{c} \xi^{2} \pm b_{\text {row }} \\
\frac{\partial \eta}{\partial x}=C_{s}+C_{c} \eta=\frac{M z^{\prime}}{C_{y a w}}-\frac{\xi F y}{C_{b e n d}}+\frac{\xi}{r_{e}} \sin (\gamma)+\frac{\partial v_{o}}{\partial x}
\end{gathered}
$$

The equations for $\frac{\partial u_{o}}{\partial x}$ and $\frac{\partial v_{o}}{\partial x}$ are given by:

$$
\frac{\partial v_{o}}{\partial x}=\frac{\partial v_{o}}{\partial x} \frac{\xi}{\gamma y} \frac{\xi}{r e} \sin (\gamma)+\left(1+{\frac{\partial v_{o}}{\partial y}}_{\gamma y}\right) \frac{\xi}{r_{e}} \gamma_{c o n}+\alpha_{p l y}
$$




$$
\begin{aligned}
& {\frac{\partial u_{o}}{\partial x}}_{\operatorname{con}(x)}=\left(1+{\frac{\partial u_{o}}{\partial x}}_{\gamma x}\right) \frac{\xi}{r_{e}} \gamma_{c o n} \\
& {\frac{\partial u_{o}}{\partial x}}_{\gamma x}=-\epsilon_{\gamma x} \\
& {\frac{\partial v_{o}}{\partial x}}_{\gamma y}=-\epsilon_{\gamma y}
\end{aligned}
$$

In the above equations the curvature due to camber and conicity is represented by

$$
\frac{\left(x^{2}-a^{2}\right)}{2 r_{e}}\left(1-\frac{\partial v_{o}}{\partial x}\right)\left(\gamma_{c o n}+\sin (\gamma)\right) .
$$

The curvature of a particular tire can be modelled appropriately by selecting proper values of $\epsilon_{\gamma x}$, and $\epsilon_{\gamma y}$. The external forces will cause translation, bending, and twisting

of the contact patch. The corresponding stiffnesses are represented by $C_{l a t}, C_{b e n d}$, and $C_{\text {yaw }}$.

\subsubsection{The Tread Simulation Program}

The Tread Simulation Program, developed by Pacejka [6], functions by dividing the lateral width of the tire contact patch into a number of belts, which are discritized into $n$ intervals that span longitudinally. The tire contact patch model is illustrated in Figure 2.10.

The belts will deform due to the level of camber, conicity, ply-steer, and external forces acting on the tire after being normally loaded, which is represented by Equation 2.19. The belt of the tire is situated above the tread touching the ground and will not deform due to sheer generated by friction. From a top view of the contact patch, a projection of the tire's belt onto the road surface will be used as a reference for the tire's tread directly below the belt. On a frictionless surface the tread and the projection of the belt will coincide with one another. A point on the belt B will begin 


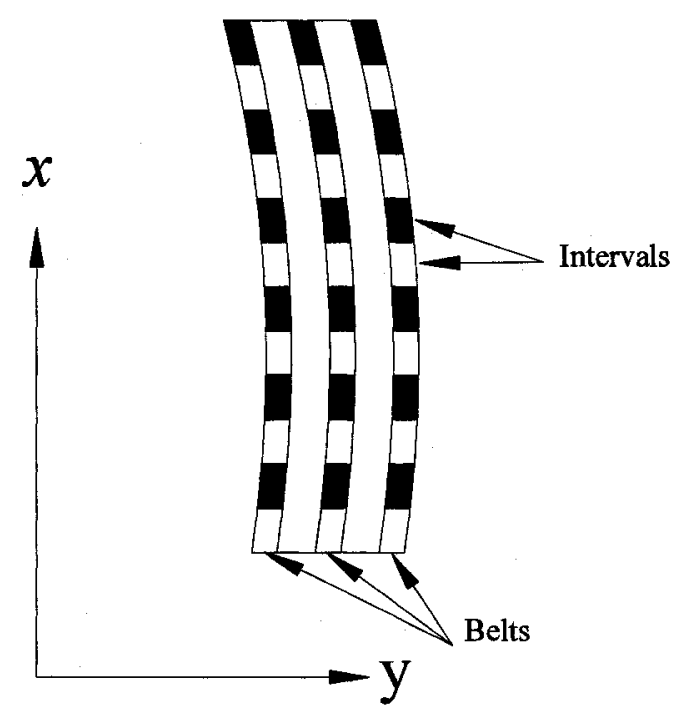

Figure 2.10: Discritization of the tire contact patch.

at the leading edge of the tire contact patch and will travel backwards $i$ intervals in $i$ time steps represented by $\triangle t$. The length travelled by point $\mathrm{B}$ in one time step is calculated by

$$
\triangle x=\frac{2 a}{n}
$$

where $a$ is half the tire's contact patch length. The length of each time step is determined by

$$
\triangle t=\frac{\triangle x}{V_{r}}
$$

The velocity of point B relative to the ground is determined by using Equation 2.17 and 2.18 by setting $\frac{\partial u}{\partial x}$ and $\frac{\partial v}{\partial x}$ to zero. The displacement vector of $\mathrm{B}, \triangle \vec{s}$, in each time step is calculated by

$$
\triangle \vec{s}=\vec{Q} \triangle t
$$

The vector $\vec{e}$ will represent the deformation of the tread relative to the projection of the belt onto the ground. Figure 2.11 illustrates the tread deformation relative to 
the belt from the perspective of an observer on the ground.

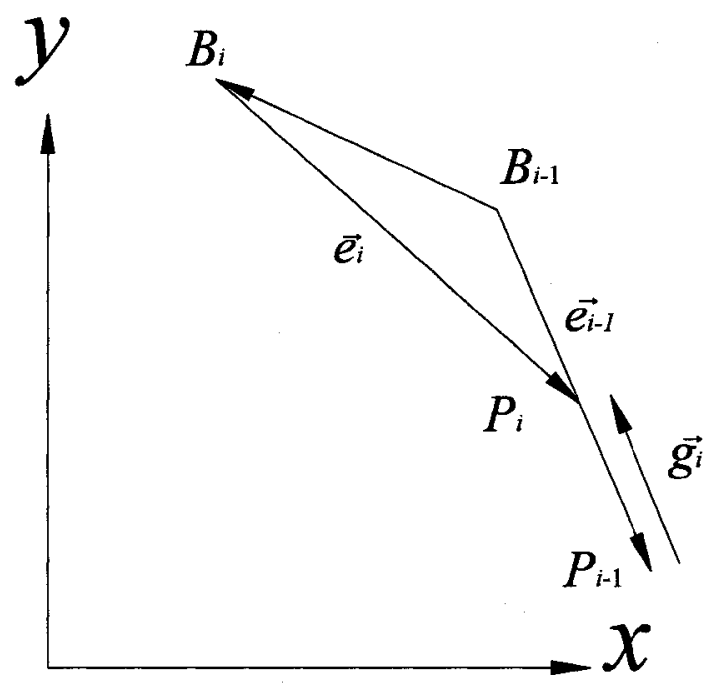

Figure 2.11: Tire tread deformation relative to the ground.

Point $\mathrm{P}$ represents a point on the tread that coincides with point $\mathrm{B}$ before deformation. When point $P$ first enters the contact zone at the leading edge, it will adhere to the ground under the force of friction. Due to the displacement of the tire and the path point $\mathrm{B}$ is forced to travel due to induced curvature, deformation between the adhered point $\mathrm{P}$ and point $\mathrm{B}$ will occur. The deformation is calculated by

$$
\vec{e}_{i}=\vec{e}_{i-1}-\triangle \vec{s}_{i}
$$

Point $\mathrm{P}$ remains fixed to the ground at the same spot until the shear force, $\vec{q}$, is large enough to overcome the force of friction. Point $\mathrm{P}$ then begins to slide with a velocity relative to the ground $\vec{g}$. The relationship between the shear force and the tread deformation is represented by

$$
\vec{q}=c_{p} \vec{e}
$$


where $c_{p}$ represents the tire tread's lateral stiffness. Coefficient $c_{p}$ can be calculated from

$$
C_{F \alpha}=2 c_{p y} a^{2}
$$

and

$$
C_{F \kappa}=2 c_{p x} a^{2}
$$

and for an isotropic tread material $c_{p x}$ will equal $c_{p y}$. Sliding will occur if

$$
\left|q_{i}\right| \leq \frac{\mu_{i} q_{z, i}}{c_{p}}
$$

where $q_{z, i}$ represents the normal pressure at point $P_{i}$. The normal pressure is assumed to have the normal force distributed in a parabolic fashion. The new equation that represents sliding is represented by

$$
\vec{e}_{i}=\vec{e}_{i-1}-\triangle \vec{s}_{i}+\vec{g}_{i}
$$

In the above equation there are two unknown quantities to be solved. The sliding velocity's magnitude is unknown; however it is known that $\vec{g}$ will be in the opposite direction to the deformation vector $\vec{e}_{i-1}$. Since the maximum shear force occurs at the onset of sliding, the magnitude of $\vec{e}$ is known, and its direction must be solved. Figure 2.12 illustrates the solution to the problem graphically. As seen on Figure 2.12 , the unknown quantities can be solved by finding the intersection between a line and a circle. This problem will present two nonlinear equations with two unknowns. The intersection can be solved by finding the root numerically or with

$$
\left|g_{i}\right|=\frac{1}{2} e_{i-1} \frac{\left(e_{x, i-1}-\triangle s_{x, i}\right)^{2}+\left(e_{y, i-1}-\triangle s_{y, i}\right)^{2}-\left|e_{i}\right|^{2}}{e_{x, i-1}\left(e_{x, i-1}-\triangle s_{x, i}\right)+e_{y, i-1}\left(e_{y, i-1}-\triangle s_{y, i}\right)}
$$




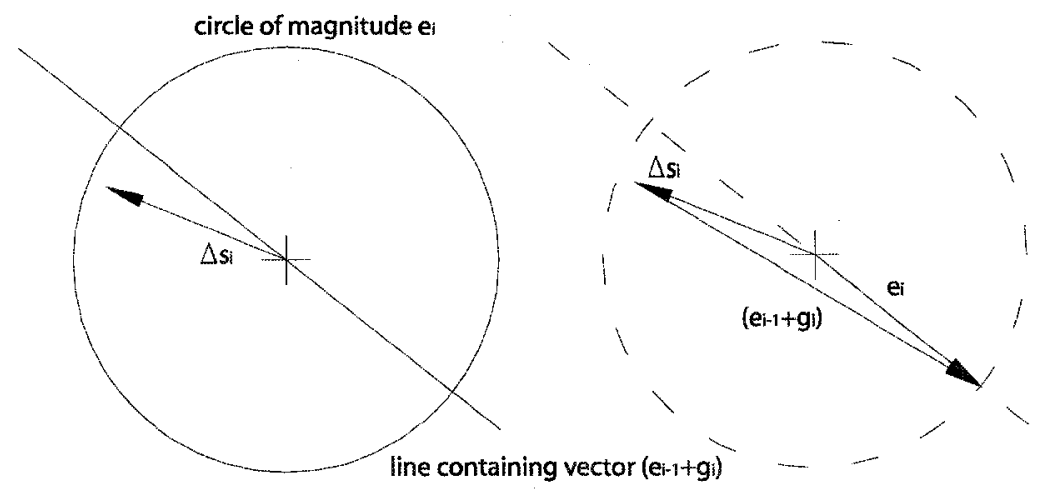

Figure 2.12: Graphical Solution to Equation 2.33

which is a simplification that relies on $\left|g_{i}\right| \ll\left|e_{i}\right|$.

\subsection{Tire Empirical Model}

The Magic Formula tire model, developed by Pacejka [6], is an empirical tire model that uses analytical equations to match the tire's behaviour observed through test results. All manner of tire behaviour can be modelled, including forces created from lateral slip, longitudinal slip, combined slip, camber inclination, and turn slip. The equations are designed in such a way that they allow easy manipulation to account for the effects of normal force. 


\subsubsection{Lateral and Longitudinal Force Model}

The lateral force caused by slip angle is modelled by

$$
F_{y \alpha}=D_{\alpha} \sin \left[C_{\alpha} \arctan \left(B_{\alpha} \alpha-E_{\alpha}\left(B_{\alpha} \alpha-\arctan \left(B_{\alpha} \alpha\right)\right)+H_{\alpha} \arctan ^{7}\left(B_{\alpha} \alpha\right)\right)\right]+S_{V y}
$$

with

$$
\alpha=\alpha_{o}+S_{H y}
$$

where $B_{\alpha}, C_{\alpha}, D_{\alpha}, E_{\alpha}$, and $H_{\alpha}$ are shaping parameters for the equation. All of the shape factors have physical meaning and will be explained further in chapter three.

The longitudinal force can be modelled in similar fashion by

$$
F_{x \kappa}=D_{\kappa} \sin \left[C_{\kappa} \arctan \left(B_{\kappa} \kappa-E_{\kappa}\left(B_{\kappa} \kappa-\arctan \left(B_{\kappa} \kappa\right)\right)\right)\right]+S_{V x}
$$

with

$$
\kappa=\kappa_{o}+S_{H x}
$$

Without the $S_{H y}$ or $S_{H x}$ offset, the force curve has an anti-symmetric shape with respect to the origin. The coefficients $\alpha_{o}$ and $\kappa_{o}$ represent the lateral and longitudinal slip variables with the absence of the horizontal shift.

\subsubsection{Aligning Torque Model}

The aligning torque is the moment produced around the tire $\mathrm{z}$-axis. There are three separate causes that produce the torque. Both the longitudinal and lateral forces can be offset from the centre of the tire creating a moment. The camber angle will affect the aligning torque in two ways. First, the camber angle will change the value of the lateral force which will cause the moment curve to be asymmetric with respect 
to $\alpha$. Finally, a residual torque will be created due to camber. The total moment is represented by

$$
M_{z}=-t F y_{y}+s F_{x}+M_{z r}
$$

where the pneumatic trail is modelled with

$$
t(\alpha)=D_{t} \cos \left[C_{t} \arctan \left(B_{t} \alpha-E_{t}\left(B_{t} \alpha_{t}-E_{t}\left(B_{t} \alpha_{t}-\arctan \left(B_{t} \alpha_{t}\right)\right)\right)\right)\right]
$$

and the moment arm for the longitudinal force is modelled with

$$
s\left(F_{y}, \gamma\right)=-\left(\frac{c_{s 1} a_{o}}{F_{z o}} F_{y}-c_{s 2} a_{o}-c_{s 3} b \gamma\right)
$$

and the residual moment due to camber is modelled with

$$
M_{z r}=D_{r} \cos \left[C_{r} \arctan \left(B_{r} \alpha\right)\right]
$$

In the model, additional shape parameter constants are represented by $c$ with subscripts refereing to the equation that they belong to.

\subsubsection{Turn Slip Model}

The rotation of an upright tire about its vertical axis is known as turn slip. Turn slip will contribute an additional velocity to every point on the contact patch apart from the exact centre. Needless to say, the effects of combined turn slip with another tire input quantity is very complicated. Therefore, the effects of turn slip on the different tire inputs will be observed through empirical results. Empirical results show that the longitudinal force will diminish with an increase of turn slip. The weighting function 
used to model this effect is

$$
\zeta_{1}=\cos \left(\arctan \left(B_{x \varphi} r_{\circ} \varphi\right)\right)
$$

where

$$
B_{x \varphi}=c_{x \varphi 1}\left(1+c_{x \varphi 2} F_{z}\right) \cos \left(\arctan \left(c_{x \varphi 3} \kappa\right)\right)
$$

The weighting factor $\zeta_{1}$ is then multiplied by $D_{\kappa}$ and $S_{v x}$ to achieve the new longitudinal force due to the combined effects of turn and longitudinal slip.

Figure 2.13 demonstrates the combined effects of a slip angle and turn slip on the lateral force. Several augmentations must be made to the original Magic Formula curve given by Equation 2.35. Similar to the effect of camber, the curve is shifted along the slip angle axis. This shift is accomplished by adding

$$
S_{H \varphi}=D_{\varphi} \sin \left[C_{\varphi} \arctan \left[B_{\varphi} r_{o} \varphi-E_{\varphi}\left(B_{\varphi} r_{o} \varphi-\arctan \left(B_{\varphi} r_{o} \varphi\right)\right)\right]\right]
$$

to Equation 2.36. The flattening of the curve is accomplished by multiplying

$$
\zeta_{2}=\cos \left[\arctan \left[c_{y \varphi 1}\left(1+c_{y \varphi 2} F_{z}\right) \cos \left(\arctan \left(c_{y \varphi 3} \tan (\alpha)\right)\right)\left(\left|r_{o}\right| \varphi+\left(c_{y \varphi 4} \sqrt{r_{o} \varphi}\right)\right)\right]\right]
$$

by the peak value $D_{\alpha}$. The value for the vertical shift $S_{V y}$ is also multiplied by $\zeta_{2}^{2}$ in order to change the overall height of Equation 2.35. Finally, the cornering force as a function of the normal force, Equation 2.61, will also change due to turn slip. The weighting function

$$
\zeta_{3}=\cos \left[\arctan \left(c_{y \varphi 5} r_{o}^{2} \varphi^{2}\right)\right]
$$

is multiplied to Equation 2.61 to accomplish the augmentation.

Turn slip will have an overall effect on the aligning torque by decreasing the 


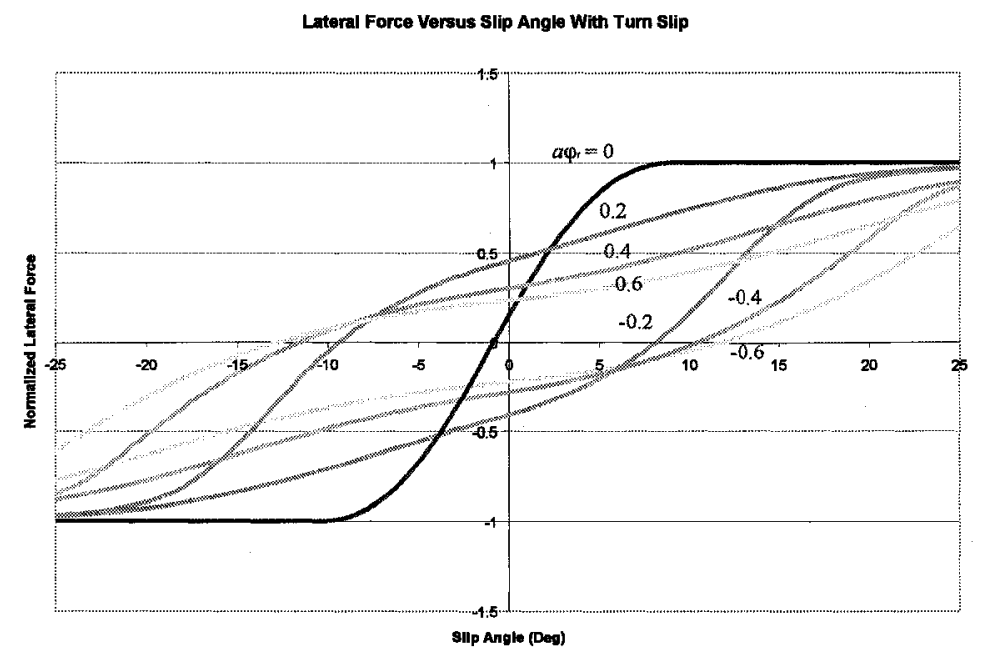

Figure 2.13: Lateral force versus slip angle with turn slip.

pneumatic trail and manipulating the residual torque. The pneumatic trail may be shortened by multiplying the weighting factor

$$
\zeta_{4}=\cos \left[\arctan \left(c_{m \varphi 1} r_{o} \varphi\right)\right]
$$

by the peak value $D_{t}$.

The peak value of the residual torque is increased by adding

$$
D_{r \varphi}=\frac{M_{z \varphi \infty}}{\sin \left(\frac{1}{2} \pi C_{r \varphi}\right)} \sin \left[C_{r \varphi} \arctan \left[B_{r \varphi} r_{o} \varphi-E_{r \varphi}\left(B_{r \varphi} r_{o} \varphi-\arctan \left(B_{r \varphi} \varphi r_{o} \varphi\right)\right)\right]\right]
$$

where

$$
M_{z \varphi \infty}=c_{m \varphi 2} \mu_{y} r_{o} F_{z} \sqrt{\frac{F_{z}}{F_{z o}}}
$$

which represents the moment generated when an upright tire is rotated about its vertical axis in the absence of translation.

The shape of the aligning torque curve gradually becomes more bell shaped with 
the increase in turn slip. To achieve this shape, $B_{r}$ is multiplied by the weighting factor

$$
\zeta_{5}=\cos \left[\arctan \left(c_{m \varphi 2} r_{o} \varphi\right)\right]
$$

\subsection{Tire Semi-Empirical Model}

A semi-empirical tire model allows for a fully developed tire model without the need for empirical testing of every conceivable situation. The normal force and the surface frictional coefficient acting on a tire has a profound effect on the tire's behaviour. Equations are presented that will determine the tire's behaviour with one set of parameters using empirical data tested with another set of parameters.

\subsubsection{The Similarity Method}

The Similarity Method tire model, developed by Pacejka [6], is a semi-empirical model created based on the observation that the force versus slip curves remain generally of the same shape under different conditions. Pacejka [6] shows that the force values resulting from different test conditions could be normalized to fit the same curve. Therefore, it is possible to develop a set of equations that will manipulate a nominal set of empirical data, generating the behaviours under the new conditions.

The first step when using the similarity method is to collect a set of data giving the relationships between lateral force versus slip angle, aligning torque versus slip angle, and longitudinal force versus longitudinal slip. The data must be collected at several different values of camber and normal force. With one set of data, all with the same normal force and frictional coefficient, the data can be fitted using the magic formula (Equations $2.35,2.37$, and 2.39). The values of the normal force $F_{z o}$ and frictional 
coefficient $\mu_{o}$ used in the data collection are declared as the nominal parameters.

The deformation, and therefore the forces, versus slip quantities are coupled with each other. This can be demonstrated mathematically by observing the deformation of a given point in the contact patch:

$$
\left[\begin{array}{l}
u \\
v
\end{array}\right]=\vec{V}_{s} \Delta t=\vec{V}_{s} \frac{(a-x)}{V_{r}}
$$

where $(a-x)$ gives the location of a point on the contact patch without the deformation added. This is a simplification of a real contact patch since it neglects curvature. Curvature would replace the $(a-x)$ term with a more complicated expression. Therefore, the input variables for the deformation are $\vec{V}_{s}$ and $V_{r}$. These variables can be represented with the slip quantities by

$$
\frac{\vec{V}_{s}}{V_{r}}=\left[\begin{array}{c}
\frac{\kappa}{1+\kappa} \\
\frac{\tan (\alpha)}{1+\kappa}
\end{array}\right]=\left[\begin{array}{c}
\sigma_{x} \\
\sigma_{y}
\end{array}\right]
$$

which uncouples the longitudinal and lateral forces by using $\sigma_{x}$ and $\sigma_{y}$ as the coupled slip input parameters. Equations 2.35, 2.37, and 2.39 can then be fitted with $\sigma_{x}$ and $\sigma_{y}$ in place of $\kappa$ and $\alpha$. The nominal curves are represented by $F_{x o}\left(\sigma_{e q}^{x}\right), F_{y o}\left(\sigma_{e q}^{y}\right)$, and $M_{z o}^{\prime}\left(\sigma_{e q}^{y}\right)$ respectively.

Observation of the lateral and longitudinal force curves reveals that both changes in normal force and changes in frictional coefficient will compress or stretch the curves proportionally with respect to the nominal values. Furthermore, the slopes at low slip parameter are altered due to the change in normal force. The equations that represent the similarity method are:

$$
F_{x}=\frac{\sigma_{x}}{|\sigma|} \frac{\mu_{x}}{\mu_{x o}} \frac{F_{z}}{F_{z o}} F_{x o}\left(\sigma_{e q}^{x}\right)
$$


with

$$
\sigma_{e q}^{x}=\frac{C_{F \kappa}\left(F_{z}\right)}{C_{F \kappa o}} \frac{\mu_{x o}}{\mu_{x}} \frac{F_{z o}}{F_{z}}|\sigma|
$$

for the longitudinal force, and

$$
F_{y}=\frac{\sigma_{y}}{|\sigma|} \frac{\mu_{y}}{\mu_{y o}} \frac{F_{z}}{F_{z o}} F_{y o}\left(\sigma_{e q}^{y}\right)
$$

with

$$
\sigma_{e q}^{y}=\frac{C_{F \alpha}\left(F_{z}\right)}{C_{F \alpha o}} \frac{\mu_{y o}}{\mu_{y}} \frac{F_{y o}}{F_{y}}|\sigma|
$$

for the lateral force, and

$$
M_{z}=\frac{\sigma_{y}}{|\sigma|} \frac{\mu_{y}}{\mu_{y o}} \frac{F_{z}}{F_{z o}} \frac{C_{F \alpha o}}{C_{F \alpha}\left(F_{z}\right)} \frac{C_{M \alpha}\left(F_{z}\right)}{C_{M \alpha o}} M_{z o}^{\prime}\left(\sigma_{e q}^{y}\right)+M_{z r}-\left(\frac{c_{s 1} a_{o}}{F_{z o}} F_{y}-c_{s 2} a_{o}-c_{s 3} b \gamma\right) F_{x}
$$

representing the aligning torque with

$$
M_{z r}=\frac{C_{M \gamma}\left(F_{z}\right)+t\left(F_{z}\right) C_{F \gamma}\left(F_{z}\right)}{1+c_{s 4} \alpha^{2}} \gamma
$$

that represents the residual moment.

The above equations all have stiffness parameters that are functions of normal force. Empirical data is required using several different normal forces in order to obtain these relationships. The relationships can be represented by

$$
\begin{gathered}
C_{F \kappa}\left(F_{z}\right)=P_{\kappa 1} \sin \left(2 \arctan \left(\frac{F_{z}}{P_{\kappa 2}}\right)\right)\left(1-P_{\kappa 3} \gamma^{2}\right) \\
C_{F \alpha}\left(F_{z}\right)=P_{\alpha 1} \sin \left(2 \arctan \left(\frac{F_{z}}{P_{\alpha 2}}\right)\right)\left(1-P_{\alpha 3} \gamma^{2}\right) \\
C_{F \gamma}\left(F_{z}\right)=P_{\gamma 1} F_{z}
\end{gathered}
$$




$$
\begin{gathered}
C_{M \alpha}\left(F_{z}\right)=P_{M \alpha 1} \sin \left(2 \arctan \left(\frac{F_{z}}{P_{M \alpha 2}}\right)\right)\left(1-P_{M \alpha 3} \gamma^{2}\right) \\
C_{M \gamma}\left(F_{z}\right)=\frac{2 b}{r_{e}} P_{M \gamma 1} F_{z}
\end{gathered}
$$

where the $P$ 's are shaping parameters. $C_{F \kappa}\left(F_{z}\right), C_{F \alpha}\left(F_{z}\right)$, and $C_{M \alpha}\left(F_{z}\right)$ are all factors that tend to increase as normal pressure increases up to a maximum point then decrease. This gives the relationship a bell shape curve. $P_{1}$ and $P_{2}$ terms are represented physically as the maximum point on the $C\left(F_{z}\right)$ and $F_{z}$ axes respectively.

\subsection{Single Contact Point Transient Tire Models}

There are many different theories and derivations of transient tire models. They range from finite element models that discritizes the entire tire, to string models that replicate the tread deformation as is the case described in Section 1.2, to fully empirical models. All have respective advantages and disadvantages. When a transient tire model is being chosen, one must choose based on how flexible the model is in representing different tires and what computational time is required to obtain solutions.

The single contact point transient tire model described in the proceeding section was chosen based on its quick calculational time and for its flexible use with different tires. The model tracks the deformation of the contact patch centre based on several first order differential equations leading to a fast computational time. The model is flexible because it uses the results from a steady state model, such as the Magic Formula and Similarity Method, to determine the behaviour of a given tire. Both qualities contribute to the model suitability for simulating a tire in real time. 


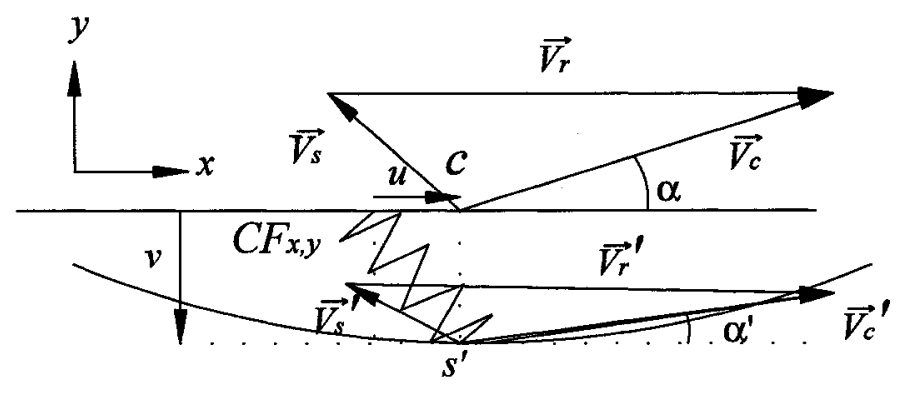

Figure 2.14: Illustration of the single contact patch tire model.

\subsubsection{Transient Deformation of the Contact Patch Centre}

A comparison will be made with the undeformed contact centre point $c$ with the deforming point $s^{\prime}$ that is situated directly beside point $c$ in the tire's lateral plane. Point $s^{\prime}$ coincides with $c^{\prime}$ s longitudinal position. Figure 2.14 illustrates the model. Point s' is constrained by two linear springs in the longitudinal and lateral directions. Their stiffness are represented as $C_{F_{x}}$ and $C_{F_{y}}$ respectively. The forces acting on the tire are

$$
F_{x}=C_{F_{x}} u
$$

and

$$
F_{y}=C_{F_{y}} v
$$

The difference between the sliding velocities of point $\mathrm{c}$ and s' will equal the rate of deformation.

$$
\frac{d u}{d t}=-\left(V_{s x}-V_{s x}^{\prime}\right)
$$




$$
\frac{d v}{d t}=-\left(V_{s y}-V_{s y}^{\prime}\right)
$$

The equation for the lateral deformation will be further developed below. Deriving the equation for the longitudinal deformation is analogous to the development of the lateral deformation.

In Equation $2.68 V_{s y}$ is the known input quantity that is determined from the side slip using Equation 2.5. An expression must be developed for $V_{s y}^{\prime}$ since it will be unknown. The lateral force can be calculated from both the cornering force multiplied by the slip angle and from the lateral stiffness multiplied by the lateral deformation. For small slip angles Equation 2.5 can be used with the terms changed to their transient counterparts in order to represent $V_{s y}^{\prime}$. An equation for $V_{s y}^{\prime}$ can then be developed as a function of the lateral deformation by

$$
\begin{gathered}
C_{F_{y}} v=C_{F_{\alpha}} \alpha^{\prime} \\
V_{s y}^{\prime}=\left|V_{c x}\right| \frac{C_{F_{y}}}{C_{F_{\alpha}}} v
\end{gathered}
$$

In the tire string model theory, $\frac{C_{F_{\alpha}}}{C_{F_{y}}}$ is often referred to as the relaxation length. This term plays an important role in the nonlinear transient model because it is a function of $\alpha^{\prime}$ and can no longer be treated as a constant. For the linear model the relaxation length will be represented by

$$
\sigma_{\alpha}=\frac{C_{F_{\alpha}}}{C_{F_{y}}}
$$

The differential equation that represents the tread deformation with time can now be expressed as

$$
\frac{d u_{\kappa}}{d t}+\frac{\left|V_{c x}\right|}{\sigma_{\kappa}} u_{\kappa}=-V_{s x}
$$




$$
\frac{d v_{\alpha}}{d t}+\frac{\left|V_{c x}\right|}{\sigma_{\alpha}} v_{\alpha}=-V_{s y}
$$

The subscripts of $\kappa$ and $\alpha$ were added to represent the deformations due to the respective input quantity.

In order to make the model relevant for larger slip angles, $V_{s y}^{\prime}$ must be left as a function of $\tan \alpha$. Also, combined slip effects can no longer be ignored. The fully nonlinear Equation 2.5 will be used to represent $V_{s y}^{\prime}$. Using Equations 2.2 and 2.4 it can be shown that

$$
V_{c x}^{\prime}=\frac{V r}{1+\kappa^{\prime}}
$$

Next Equation 2.73 can be represented as

$$
\frac{d v_{\alpha}}{d t}+\frac{V r}{1+\kappa^{\prime}} \tan \left(\alpha^{\prime}\right)=-V_{s y}
$$

Equation 2.76 can be changed to account for $C_{F_{\alpha}}$ as a function of $\alpha^{\prime}$ or as a function of $\sigma_{y}^{\prime}$, the theoretical lateral slip that accounts for the $\alpha / \kappa$ coupling. The relaxation length can then be represented as

$$
\sigma_{\alpha}^{*}=\frac{1}{C_{F_{y}}} \frac{F_{y}}{\sigma_{y}^{\prime}}
$$

The differential equations can now be represented as:

$$
\frac{d u_{\kappa}}{d t}+\frac{\left|V_{r}\right|}{\sigma_{\kappa}^{*}} u_{\kappa}=-V_{s x}
$$

and

$$
\frac{d v_{\alpha}}{d t}+\frac{\left|V_{r}\right|}{\sigma_{\alpha}^{*}} v_{\alpha}=-V_{s y}
$$

To solve Equations 2.77 and 2.78, the equation must be discritized in time. The 


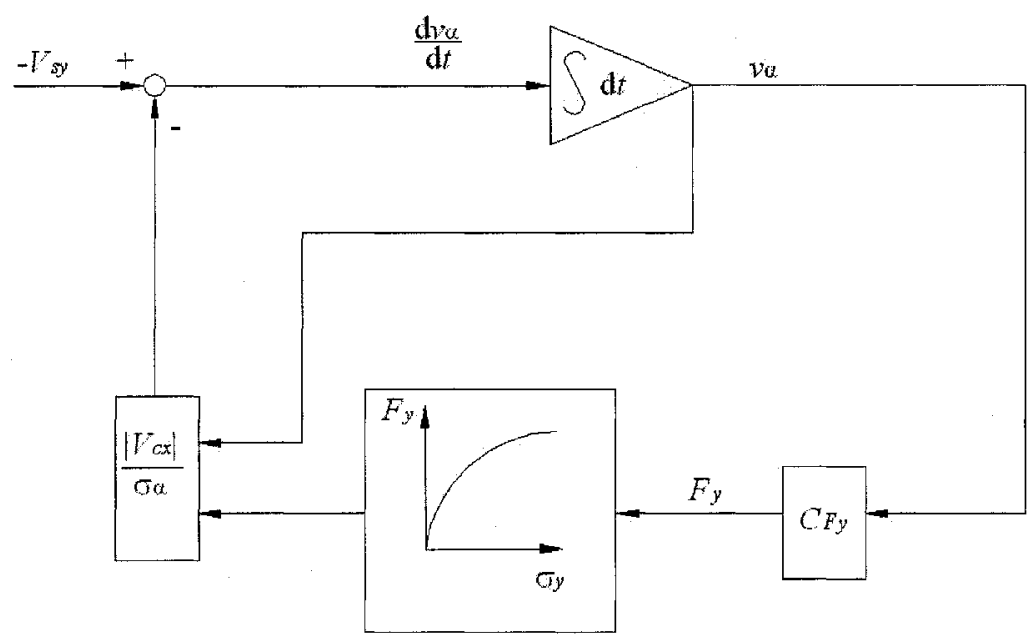

Figure 2.15: Determining the lateral deflection from an $\alpha$ input.

analysis begins with a set of initial conditions determined by $\alpha$ and $\kappa$. The solution for the linear differential equation is used for the first step in time. The force is determined with Equation 2.66. The steady state model is used to obtain $\sigma_{y}^{\prime}$ from the curve of $F_{y}$ versus $\sigma_{y}^{\prime}$. Coefficient $\sigma_{\alpha}^{*}$ is then updated by Equation 2.76. The process is repeated for the new time step using the updated parameters. Figure 2.15 shows a block diagram of this procedure.

A camber input will shift the lateral force versus slip angle graph, increasing or decreasing the force at a given slip angle. The shift can be accomplished by adding a proportional slip angle due to camber to the actual slip angle. This can be represented mathematically as

$$
\alpha^{*}=\alpha+\frac{C_{F_{\gamma}}\left(F_{z}\right)}{C_{F_{\alpha}}\left(F_{z}\right)} \gamma
$$

where $\alpha^{*}$ is the combined slip angle containing both the side slip and camber input. Coefficient $\alpha^{*}$ can be placed into Equation 2.5, which may be divided into the slip angle caused by actual lateral slip and the slip angle influenced by the camber angle. Equation 2.78 will still be used to calculate the influence of side slip and a 


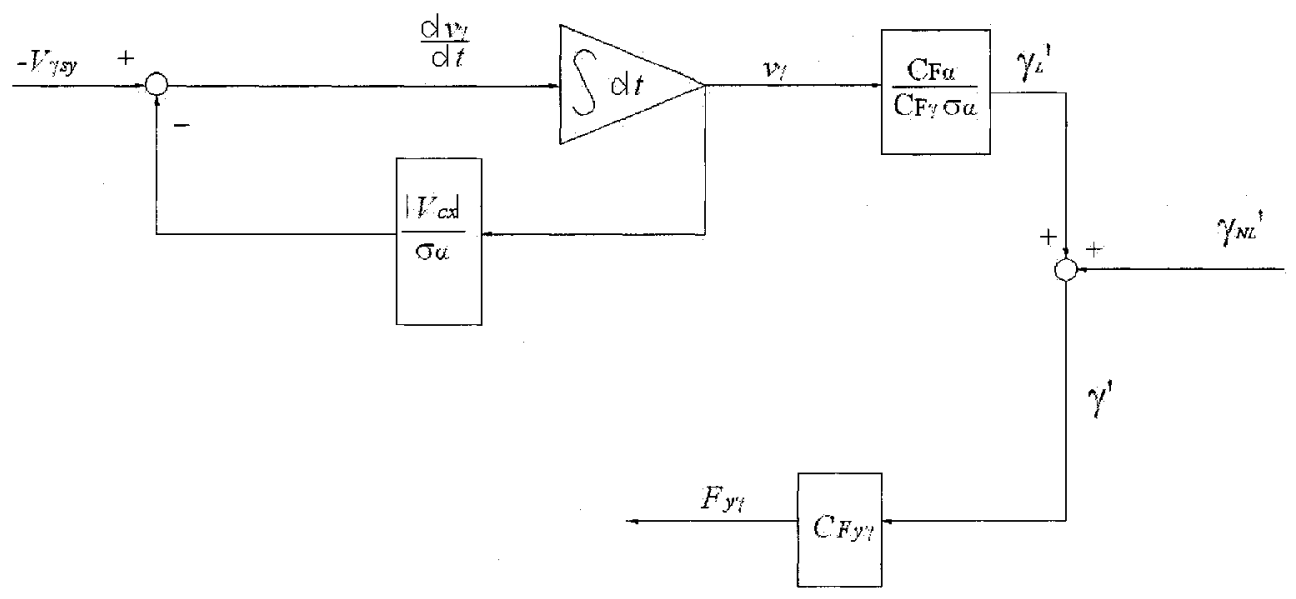

Figure 2.16: Determining the lateral deflection from a $\gamma$ input.

new expression must be developed to account for the camber input. In most applications, the tangent term of Equation 2.5 can be linearized because the camber angle is kept small; and converting it to a proportional slip angle further reduces the value. Therefore, a similar equation to Equation 2.73 will be obtained resulting in

$$
\frac{d v_{\gamma}}{d t}+\frac{\left|V_{c x}\right|}{\sigma_{\alpha}} v_{\gamma}=-V_{\gamma s y}
$$

where $-V_{\gamma s y}$ represents the lateral shift due to camber. Experiments performed by Higuchi and Pacejka [23] show that there is an immediate non-lagging increase of $-V_{\gamma s y}$ due to camber input. This was accounted for by the curvature change of the contact patch that takes place at the same rate as the tire inclination. The rate of inclination is often considered as instantaneous, and as a result, $-V_{\gamma s y}$ will be divided into a lagging and non lagging portion. The complete expression for $-V_{\gamma s y}$ is represented as

$$
-V_{\gamma s y}=\left(1-\varepsilon_{N L}\right) \frac{C_{F_{\gamma}}\left(F_{z}\right)}{C_{F_{\alpha}}\left(F_{z}\right)}\left|V_{c x}\right| \gamma
$$


Once $v_{\gamma}$ is calculated, the lagging camber angle can be obtained by

$$
\gamma_{L}^{\prime}=\frac{C_{F_{\gamma}}\left(F_{z}\right)}{C_{F_{\alpha}}\left(F_{z}\right)} \frac{v_{\gamma}}{\sigma_{\alpha}}
$$

The total transient camber angle becomes

$$
\gamma^{\prime}=\gamma_{L}^{\prime}+\varepsilon_{N L} \gamma
$$

and the lateral force will equal

$$
F_{y \gamma}=C_{F_{\gamma}} \gamma^{\prime}
$$

The computational procedure is illustrated in Figure 2.16. 


\section{Chapter 3}

\section{Tire Model Development}

The semi-empirical model was designed using the theory presented in Chapter 2. The empirical tire data required by the model was collected through tire testing and by computer simulation with the use of the TreadSim program developed by Pacejka [6]. The data was used for the selection of the tire parameters required by the Magic Formula tire model.

\subsection{Empirical Testing}

Force data was collected on a McCreary Airtrac 6.00-6 8 ply tire using the Carleton University tire testing facility. As part of this project, the tire facility was reconfigured such that it could be used to study aircraft tires on a simulated flight deck surface rather than traction studies of off-road vehicle tires for which it was originally designed. The Airtrac 6.00-6 tire is an 16 inch diameter tire commonly used on small aircraft, and is similar to the tail wheels used on the Canadian Forces Sea King helicopter.

The testing facility was originally designed to test longitudinal slip of large offroad vehicle tires by Chen [24] and Wu [25]. Retrofitting of the tire testing facility 


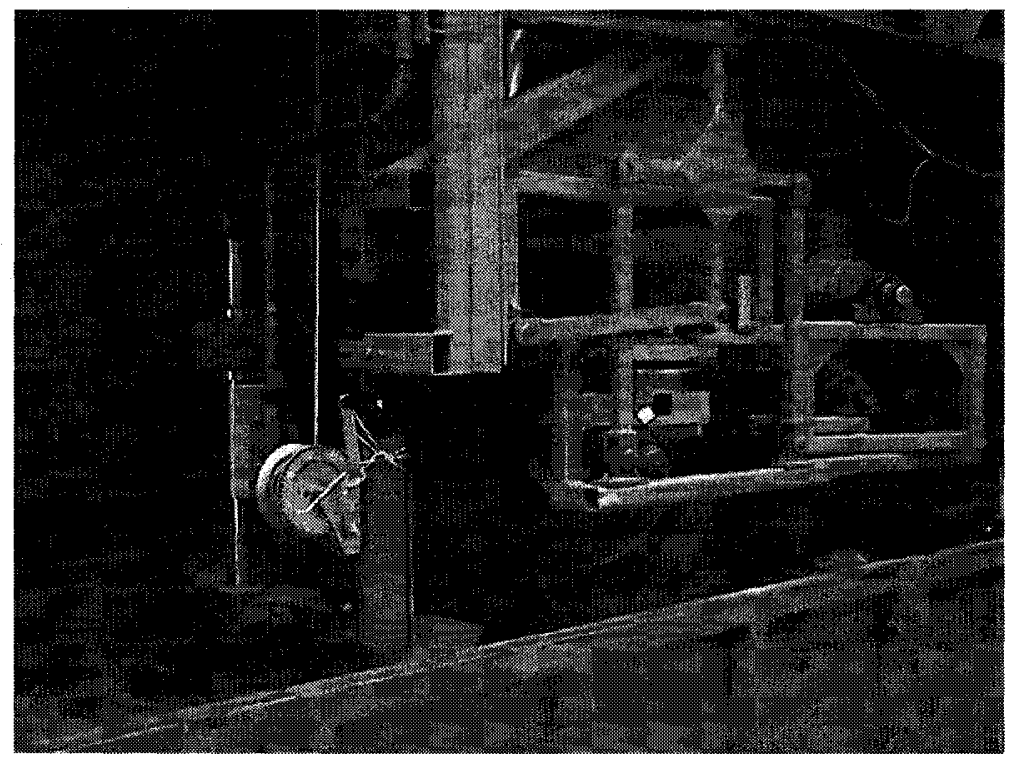

Figure 3.1: Previous setup of the tire test facility.

was required for the helicopter tail wheel simulated flight deck research. A non-skid ship deck test surface was added and a new tire mount was fabricated. The new tire mount was designed to hold the tire at a desired side slip orientation. This allowed for testing of the lateral force versus side slip characteristics of the tire. The normal force and camber angle could also be varied while collecting the lateral force data. The test facility also collected the longitudinal force and aligning moment of the tire.

The data acquisition and instrumentation had to be created, installed, and calibrated for the new batch of tire testing application. The development of the tire test facility and the test results will be presented subsequently.

\subsubsection{Tire Test Facility Setup}

Figure 3.1 illustrates the tire test facility in the previous configuration. Large off-road vehicle tires could be traversed along a sand bed with a set value of longitudinal slip. The lateral and longitudinal force data was collected by two load cells located in steel casings above the tire axis. The amount of longitudinal slip was controlled by varying 


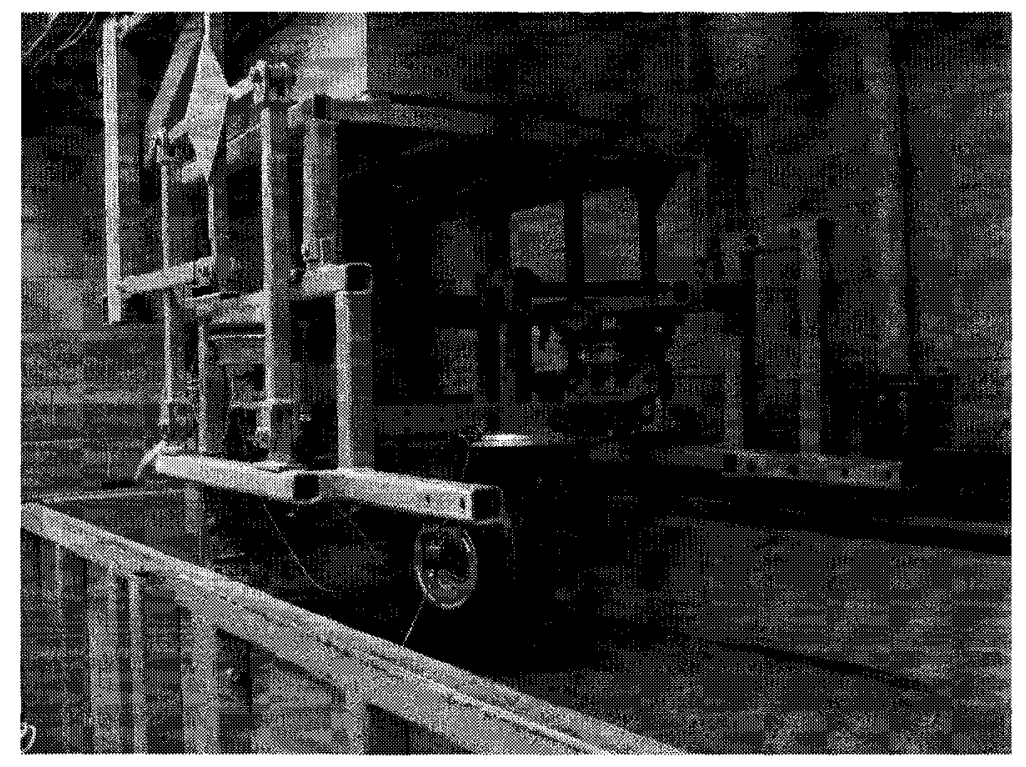

Figure 3.2: Oblique view of the tire test facility configured for aircraft tire testing.

the slip rings on a mechanism located behind the tire.

Figures 3.2 and 3.3 illustrate the new setup of the tire test facility with the new deck and tire mount installed.

The deck non-skid coating is the new standard paint for the Canadian Armed Forces. The coefficient of friction was tested using the standards presented in STANAG 1278 [26] and is vary similar to the old Canadian Military standards presented in MILC-24667A [27]. The procedure requires a sample of tire tread, attached to a steel block, be traversed across the non-skid surface while recording the average steady state shear force. The standard [26] only requires the use of a spring scale. For increased accuracy a load cell was attached to the tug string in order to record the shear force using a computer data acquisition. Five tests were required with the block traversing parallel and perpendicular to the tire tread. The test setup is illustrated in Figures 3.4 and 3.5.

When the paint surface is applied, lumps of paint gather on the roller which then form ridges perpendicular to the rolling direction. As a result, the friction 


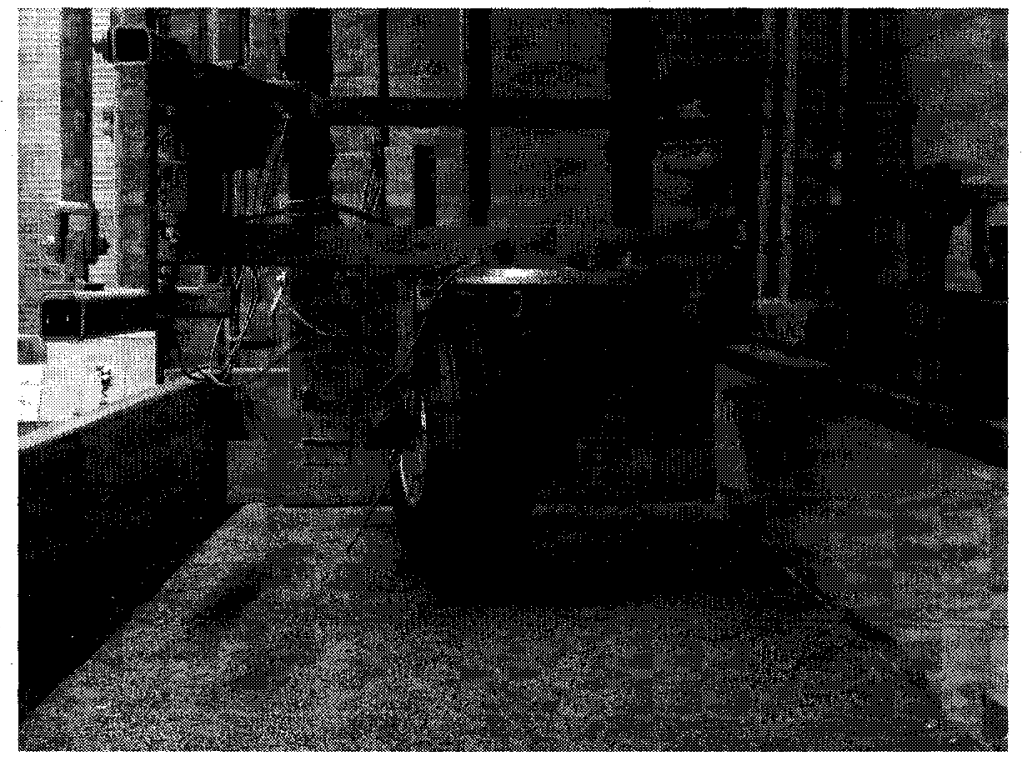

Figure 3.3: Longitudinal view of the tire test facility configured for aircraft tire testing.

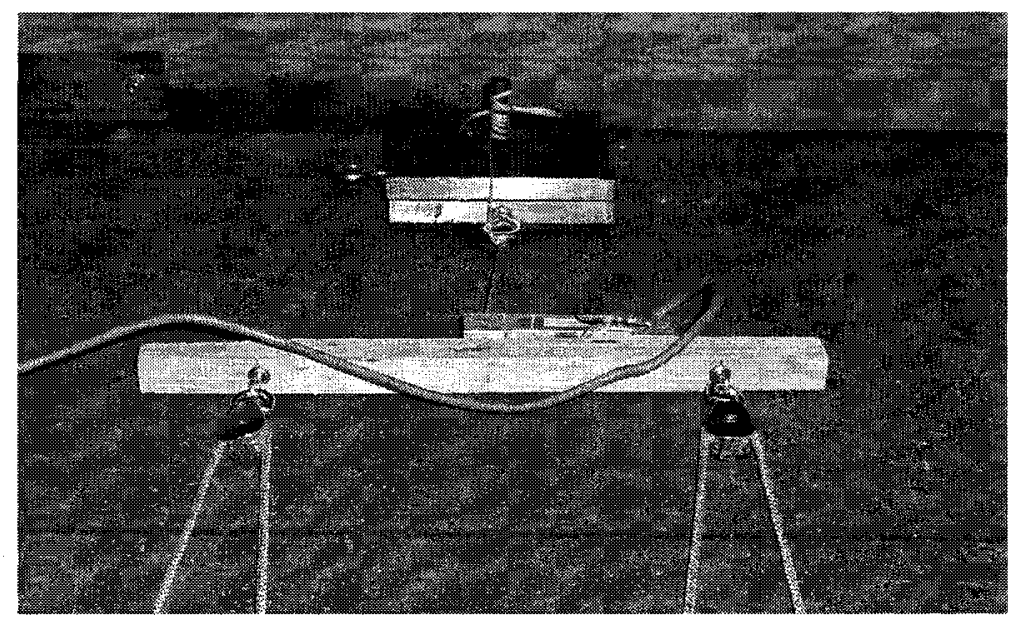

Figure 3.4: Friction test apparatus, oblique view. 


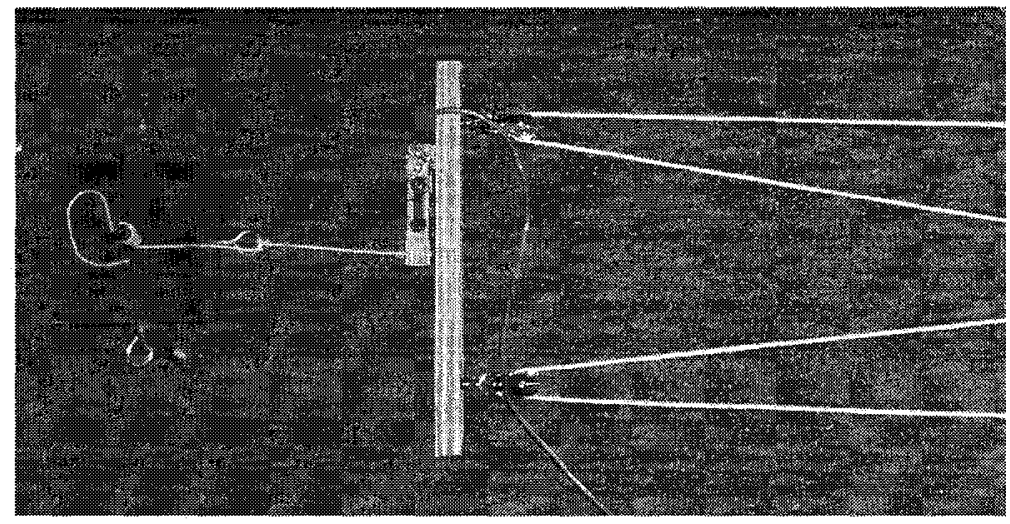

Figure 3.5: Friction test apparatus, side view.

test procedure was carried out twice, once in the direction parallel to the ridges and once in the direction perpendicular to the ridges. Table 3.1 shows the results of the experiment. Note that there are four sets of tests. The $\mathrm{X}$ is used to represent the block traversing across the paint in the test facility's longitudinal direction (the view of Figure 3.3) and the Y represents traversing along the test facility's lateral direction (as seen in Figure 3.4). For each test, the normal force on the block was 3045g. The ridges run in the lateral direction. The columns from left to right are: the average shear force on the block, the standard deviation of the average force, the average coefficient of friction for the test, the standard deviation of the coefficient of friction for the individual test, and the number of samples used in the calculations.

The test results show that there is only a small discrepancy between the tire traversing with the tread perpendicular or parallel to the travel path. The average coefficient of friction when the block was travelling against the ridge was 1.14 and with the block travelling parallel to the ridge it was 1.20 .

The tire mount was designed to test a large range of slip angles. The mount allows the tire to have a slip angle up to $45^{\circ}$. Figure 3.6 shows the tire mount set at $0^{\circ}$ slip and Figure 3.7 shows it set at $45^{\circ}$ slip. 
Table 3.1: Results of the non-skid deck friction tests

\begin{tabular}{|c|c|c|c|c|c|}
\hline Test & $\begin{array}{l}\text { Avg. Force } \\
\text { (g) }\end{array}$ & $\begin{array}{l}\text { St. Dev. } \\
\text { (g) }\end{array}$ & Coef. of Friction & St. Dev. & Num. of Samples \\
\hline X Perp to Tread t1 & 3634.92 & 788.67 & 1.19 & 0.26 & 442 \\
\hline X Perp to Tread t2 & 3628.52 & 413.06 & 1.19 & 0.14 & 438 \\
\hline X Perp to Tread t3 & 3479.85 & 714.86 & 1.14 & 0.23 & 301 \\
\hline X Perp to Tread t4 & 3349.43 & 446.54 & 1.10 & 0.15 & 371 \\
\hline X Perp to Tread t 5 & 3371.66 & 500.81 & 1.11 & 0.16 & 349 \\
\hline CF Average & & & 1.15 & & \\
\hline CF Average SDev & & & 0.04 & & \\
\hline Test & $\begin{array}{l}\text { Avg. Force } \\
\text { (g) }\end{array}$ & $\begin{array}{l}\text { St. Dev. } \\
\text { (g) }\end{array}$ & Coef. of Friction & St. Dev. & Num. of Samples \\
\hline X Par to Tread t1 & 3371.66 & 237.69 & 1.11 & 0.08 & 544 \\
\hline X Par to Tread t2 & 3215.30 & 334.61 & 1.06 & 0.11 & 489 \\
\hline X Par to Tread t3 & 3564.87 & 396.01 & 1.17 & 0.13 & 275 \\
\hline X Par to Tread t4 & 3595.78 & 287.48 & 1.18 & 0.09 & 411 \\
\hline X Par to Tread t5 & 3577.18 & 273.71 & 1.17 & 0.09 & 582 \\
\hline CF Average & & & 1.14 & & \\
\hline CF Average SDev & & & 0.06 & & \\
\hline Test & $\begin{array}{l}\text { Avg. Force } \\
\text { (g) }\end{array}$ & $\begin{array}{l}\text { St. Dev. } \\
\text { (g) }\end{array}$ & Coef. of Friction & St. Dev. & Num. of Samples \\
\hline Y Perp to Tread t1 & 3692.82 & 328.71 & 1.21 & 0.11 & 403 \\
\hline Y Perp to Tread t2 & 3672.61 & 297.59 & 1.21 & 0.10 & 499 \\
\hline Y Perp to Tread t3 & 3710.33 & 320.03 & 1.22 & 0.11 & 370 \\
\hline Y Perp to Tread t4 & 3713.05 & 301.04 & 1.22 & 0.10 & 342 \\
\hline Y Perp to Tread t5 & 3681.22 & 270.62 & 1.21 & 0.09 & 382 \\
\hline CF Average & & & 1.21 & & \\
\hline CF Average SDev & & & 0.01 & & \\
\hline Test & $\begin{array}{l}\text { Avg. Force } \\
(\mathrm{g})\end{array}$ & $\begin{array}{l}\text { St. Dev. } \\
(\mathrm{g})\end{array}$ & Coef. of Friction & St. Dev. & Num. of Samples \\
\hline Y Par to Tread t1 & 3630.26 & 192.84 & 1.19 & 0.06 & 661 \\
\hline Y Par to Tread t2 & 3622.00 & 209.55 & 1.19 & 0.07 & 453 \\
\hline Y Par to Tread t3 & 3649.79 & 229.15 & 1.20 & 0.08 & 533 \\
\hline Y Par to Tread t4 & 3643.56 & 212.99 & 1.20 & 0.07 & 469 \\
\hline Y Par to Tread t5 & 3659.99 & 209.15 & 1.20 & 0.07 & 462 \\
\hline CF Average & & & 1.20 & & \\
\hline CF Average SDev & & & 0.01 & & \\
\hline CF Avg X & 1.14 & & & & \\
\hline CF Avg Y & 1.20 & & & & \\
\hline
\end{tabular}




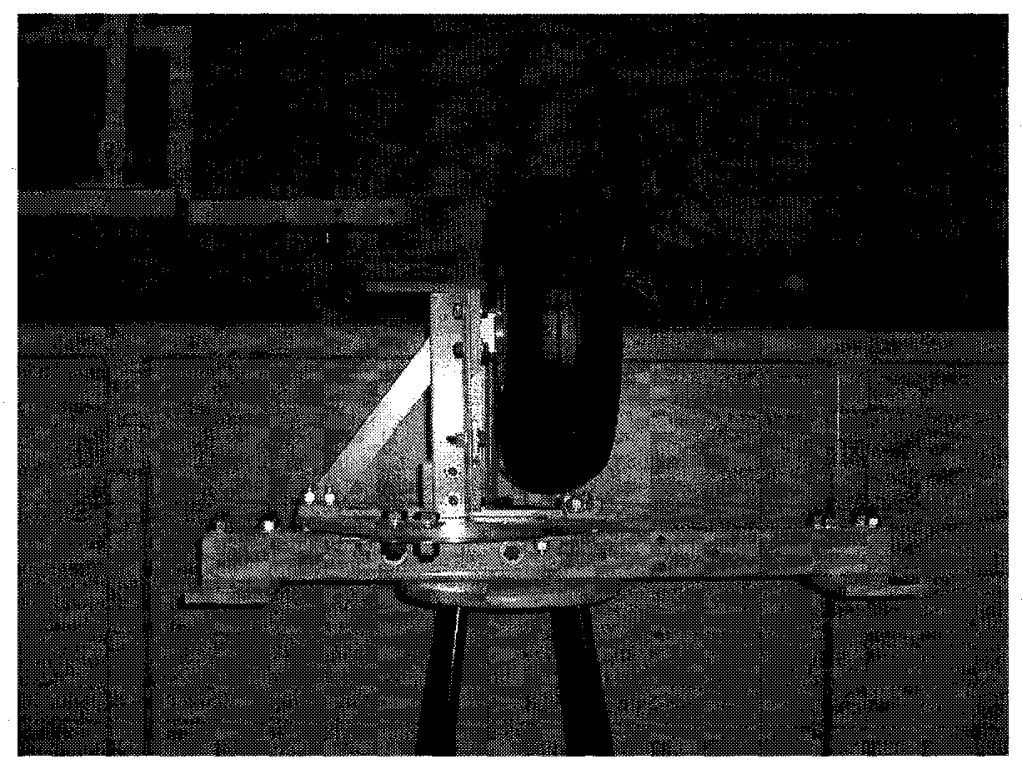

Figure 3.6: Tire mount set at $0^{\circ}$ slip angle.

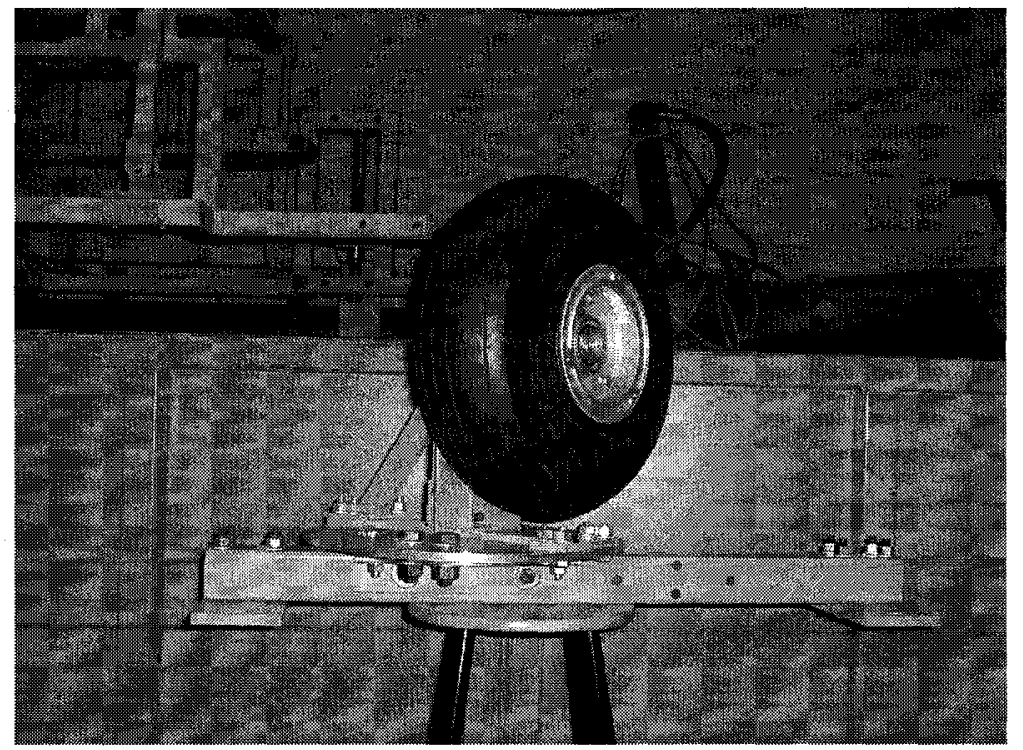

Figure 3.7: Tire mount set at $45^{\circ}$ slip angle. 


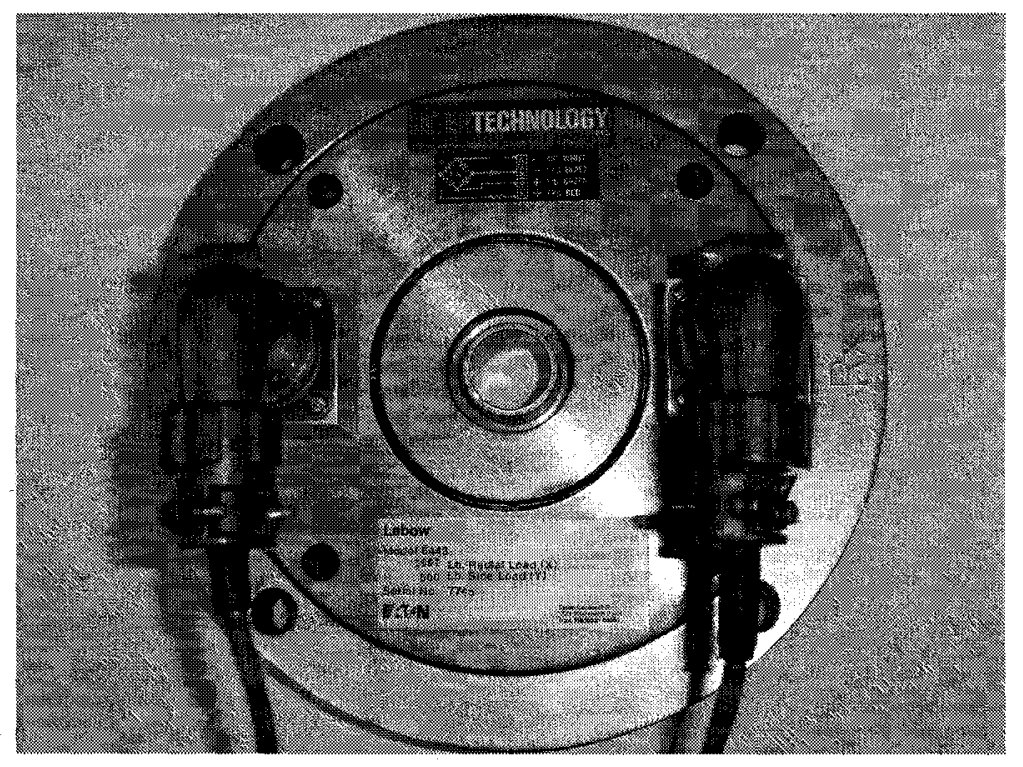

Figure 3.8: X-Y load cell used to measure tire shear forces.

\subsubsection{Instrumentation and Data Acquisition}

The tire test facility implements several different types of load cells and potentiometers to collect the experimental data. Through the use of two x-y load cells, the tire's lateral force, longitudinal force, and aligning torque are acquired. The load cells are mounted on the two attachment points connecting the tire mount to the carriage as can be observed in Figure 3.3. An axial load cell mounted under the deck and equipped with a pressure plate can measure the static normal force of the tire. There are two potentiometers mounted on the test apparatus. One of the potentiometers is mounted next to the tire and is used to measure tire lateral deflection as can be observed on Figure 3.3. The other potentiometer is used to measure carriage displacement. Figures 3.8 through 3.10 show the $x-y$ load cell, the axial load cell, and the displacement potentiometer respectively.

The instruments were calibrated though shunt calibration, axial load testing, and dead weight load testing. Each individual load cell and potentiometer were calibrated after the retrofitting of the tire testing facility. Shunt calibration is performed by 


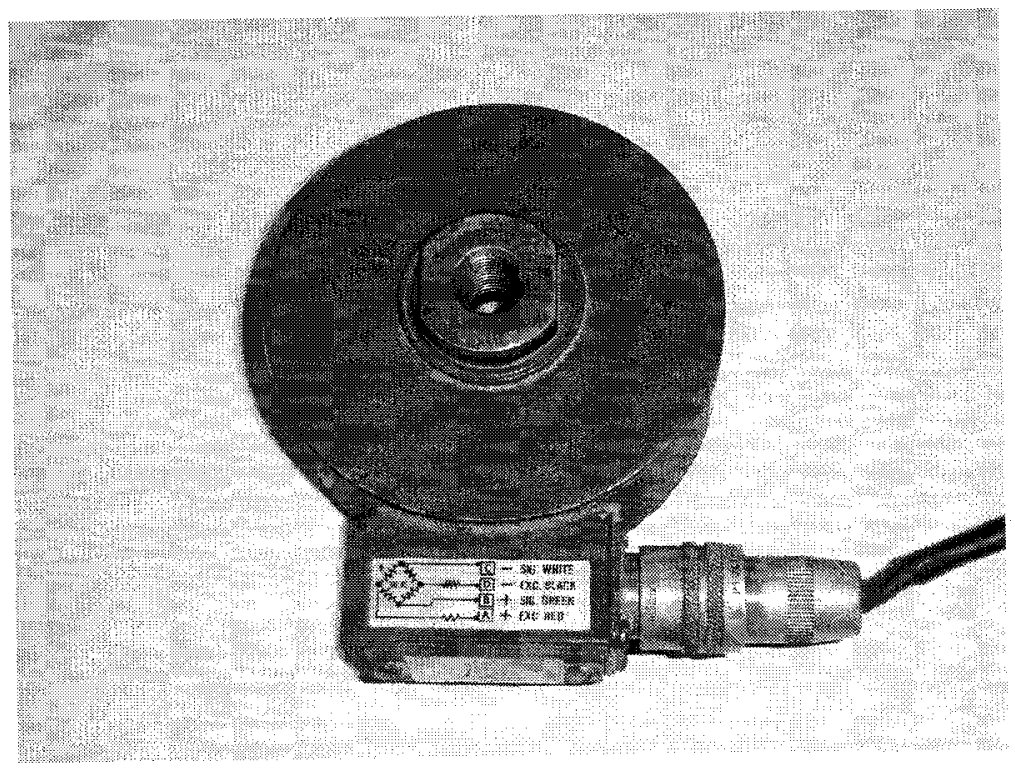

Figure 3.9: Axial load cell used to measure tire static normal force.

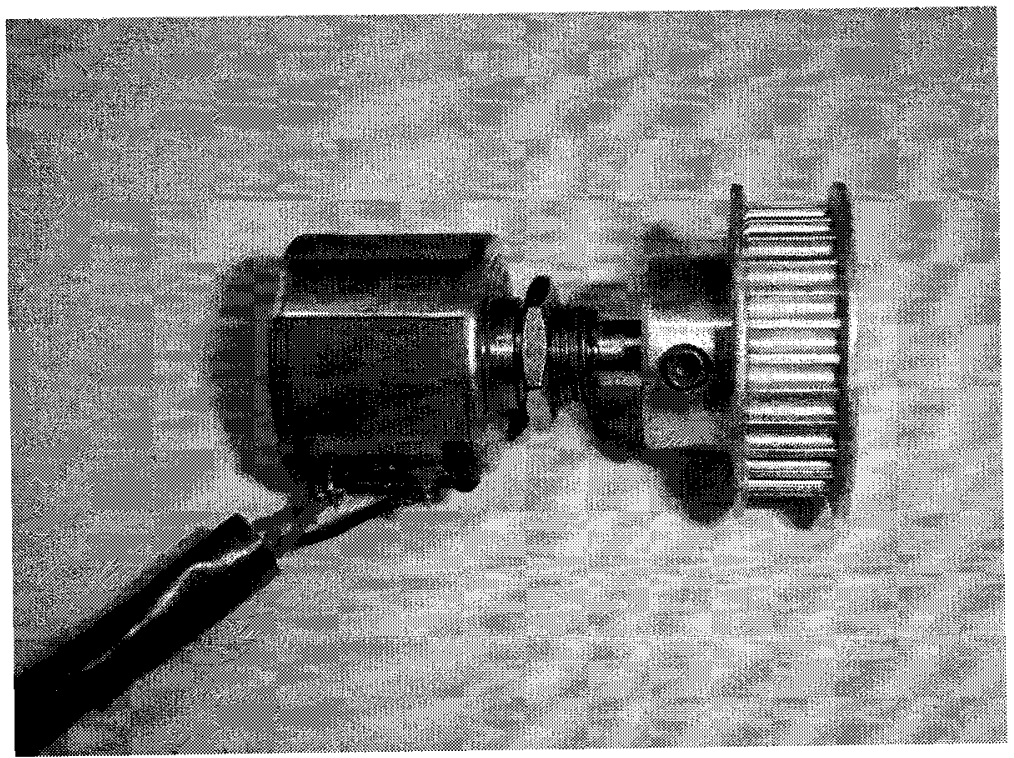

Figure 3.10: Potentiometer used to measure carriage displacement. 


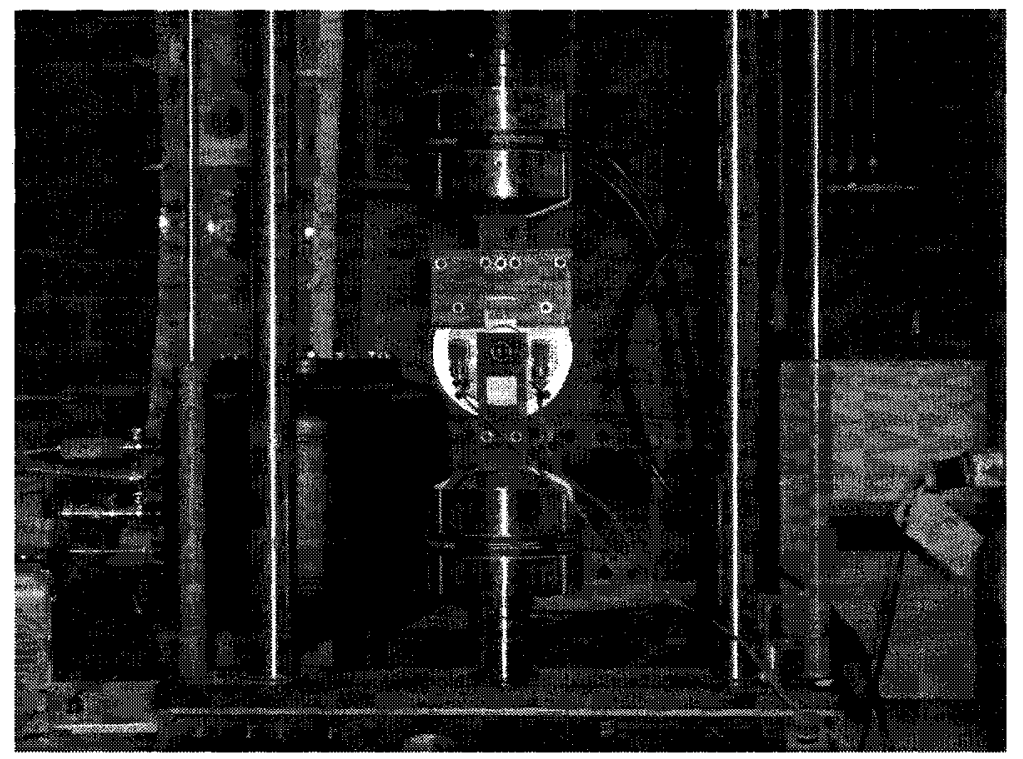

Figure 3.11: X-Y load cell mounted in the axial load testing machine.

placing a resistor across the bridge of the load cell. This creates a voltage increase and represents a load. The resistor is provided by the manufacture who tests the load cell and records the actual load that corresponds to the shunt resistor's voltage. Using this point and the nominal load, the load cell can be roughly calibrated. The wire running from the load cell to the computer adds a small amount of resistance and therefore the load cells should be re-calibrated upon installation since the slope of the load versus voltage line might differ slightly from the shunt calibration. An axial load testing machine was used to test the axial load cells up to their maximum capacity. Figure 3.11 shows an $\mathrm{X}-\mathrm{Y}$ load cell mounted in the axial load testing machine. Dead weights were used to test the smaller amplitude of the loading since the axial loading machine was designed for large loads. Once the load cells were mounted in the tire test facility a final test was performed to ensure accuracy. A second independent load cell was used to compare the measurement of the fully-mounted load cells. Appendix A shows the results of the calibration tests.

The data acquisition board is a Texas Instruments PC-LMP-16PmP. A scan rate of 
4000 readings per second was used. There were no high frequency signals being read, therefore a high scan rate was not required. The scan rate was deemed appropriate by running identical tests at higher scan rates and observing that there was no difference in the data. The computer would read 20 measurements and then record an average of the measurements. This reduced the number of recorded data points to 200 points per second. The amplifier of the PC-LMP-16PmP has a 12 bit analogue to digital converter.

\subsubsection{Tire Test Experiment and Results}

The goal behind testing the helicopter tail wheel was to obtain typical characteristics of a helicopter tire interacting with the non-skid ship deck surface. The empirical data is used to generalize the characteristics of a helicopter tire, and with the computer simulation's semi-empirical dynamic tire model. The Similarity Method requires tire data from three sets of tests performed with different normal forces. While the Magic Formula tire model requires several tests to be performed with varying camber angle. Tire longitudinal force, lateral force, and aligning torque versus slip angle data was collected at three normal load settings. For two of the tests the camber angle was also varied three times. A map of the tire steady state modelling is shown in Figure 3.12. The lateral force and aligning torque collected during the experiments are presented in Figures 3.13 through 3.18 .

Force measurements were recorded at $1^{\circ}$ slip angle intervals between $-1.2^{\circ}$ and $14.8^{\circ}$ for the first two sets of data collected with normal loadings of $351 \mathrm{lb}$ and $509 \mathrm{lb}$ respectively. These primary sets of data were used to fit the TreadSim model, the Similarity Method model, and the Magic Formula model. In the Magic Formula model three sets of points are required for fitting the cornering stiffness curve versus normal load. Therefore, a third subset of data was collected at a $591 \mathrm{lb}$ normal 


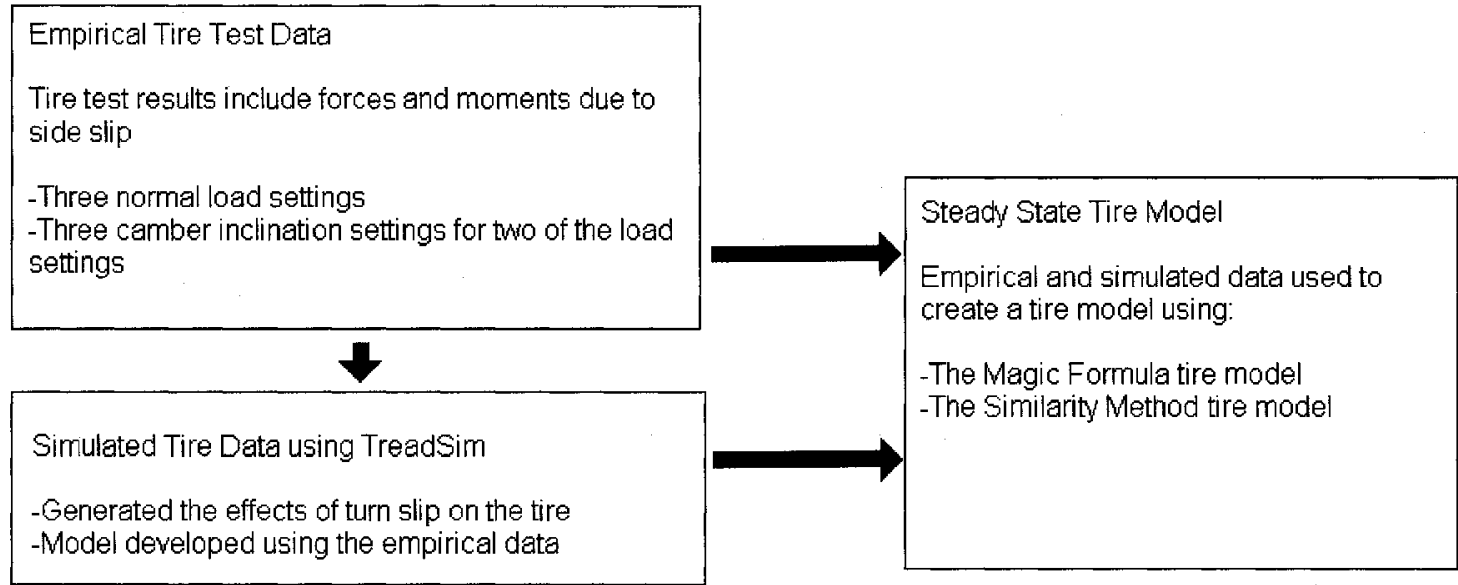

Figure 3.12: A map of the steady state tire modelling.

loading with specific points selected based on requirements for calibrating the Magic Formula model.

The shapes of the lateral force versus slip angle curves correlate well with what is typically expected of a tire undergoing lateral slip. The aligning moment curve demonstrated characteristics typically experienced by a tire undergoing both longitudinal and lateral slip. These characteristics are described by Pacejka [6]. The aligning torque will increase when there is a positive slip angle and decrease when there is a negative slip angle due to the longitudinal slip of a braking tire. Due to constraints, the test apparatus caused a small amount of interference with the force on the tire acting in the travelling direction of the tire test apparatus. This error mostly contributed to the longitudinal force acting on the tire. This caused the small magnitude of longitudinal force to behave in a erratic manner. The interference did not appear to have a large affect on the lateral force versus slip angle results. However, it did cause an undesirable spread of results for the aligning torque. The accuracy of the lateral force curve is of greater importance when compared with the aligning moment curve since the dynamic helicopter model will experience moments of greater magnitude about its mass centroid due to the lateral force generated by the tires compared to 


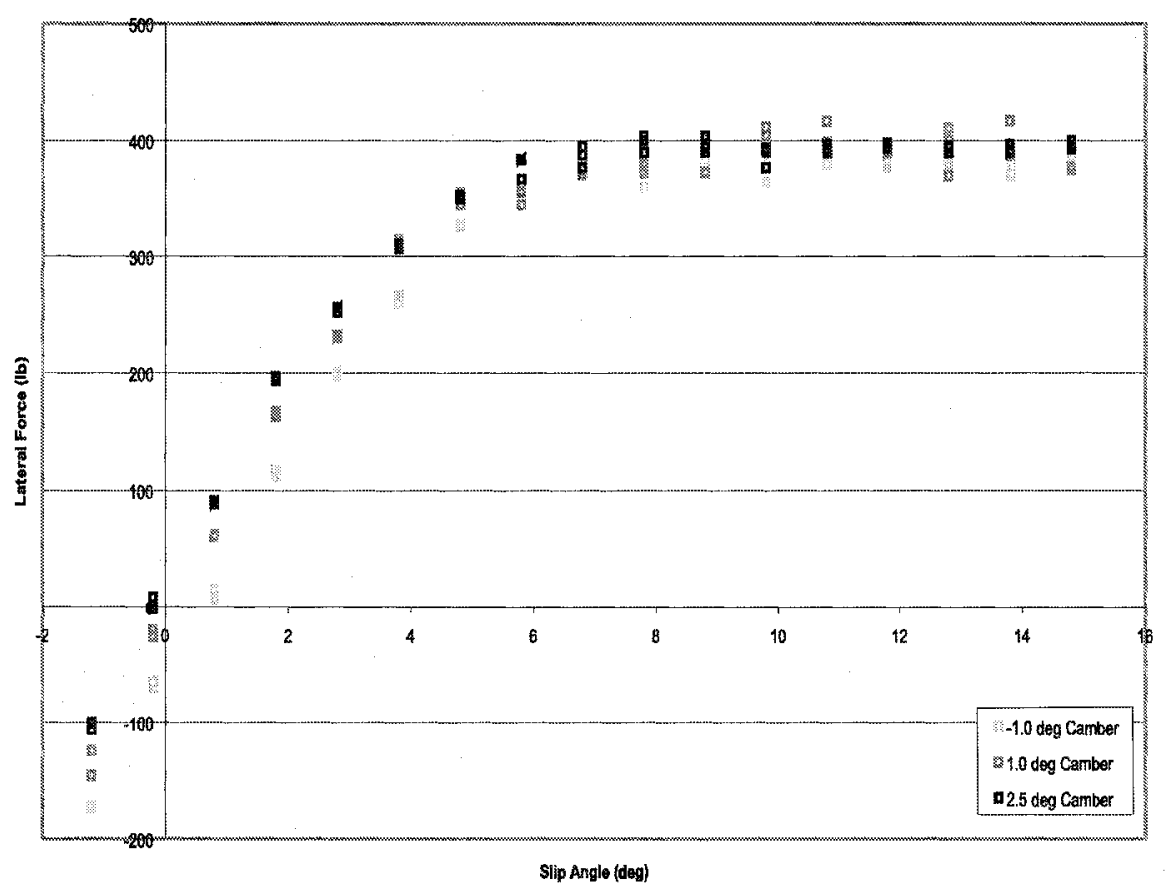

Figure 3.13: Lateral force versus slip angle empirical test results with $351 \mathrm{lb}$ normal load on an Airtrac 6.00-6 8 ply tire for various camber angles.

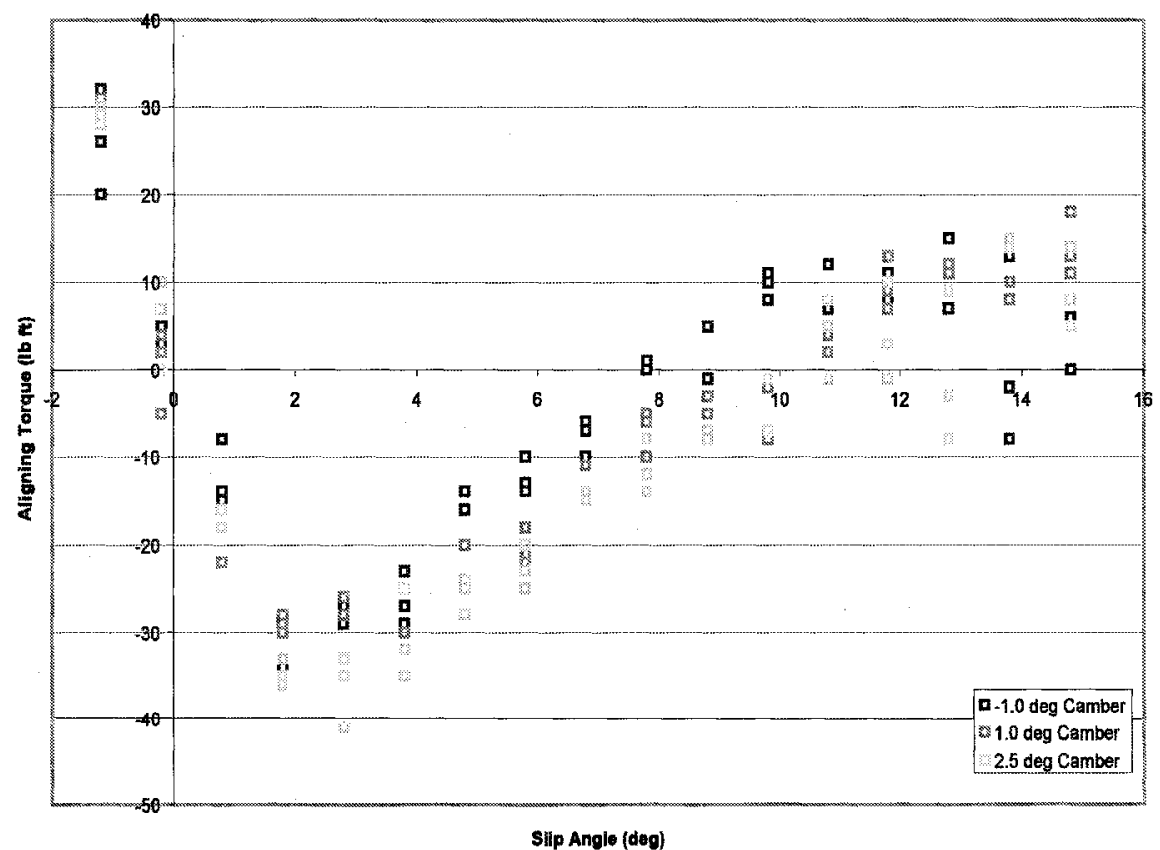

Figure 3.14: Aligning torque versus slip angle empirical test results with $351 \mathrm{lb}$ normal load on an Airtrac 6.00-6 8 ply tire for various camber angles. 


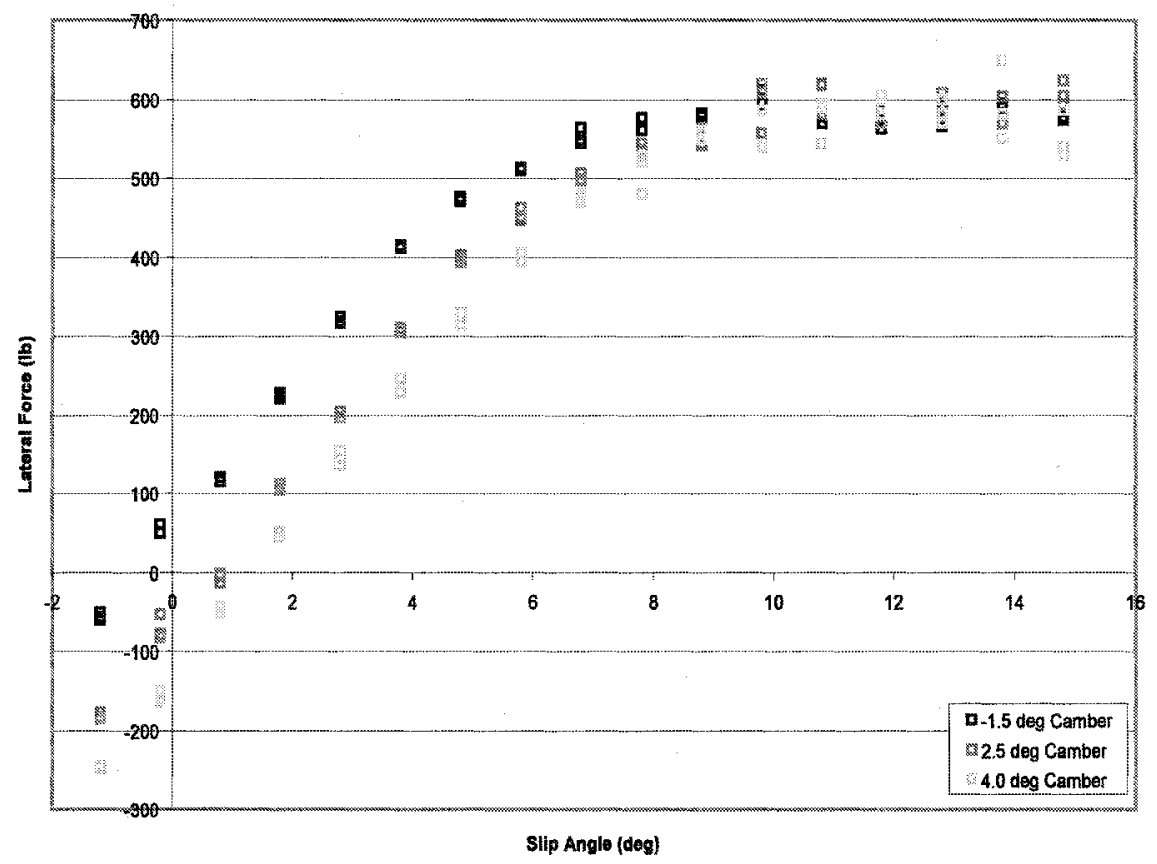

Figure 3.15: Lateral force versus slip angle empirical test results with $509 \mathrm{lb}$ normal load on an Airtrac 6.00-6 8 ply tire for various camber angles.

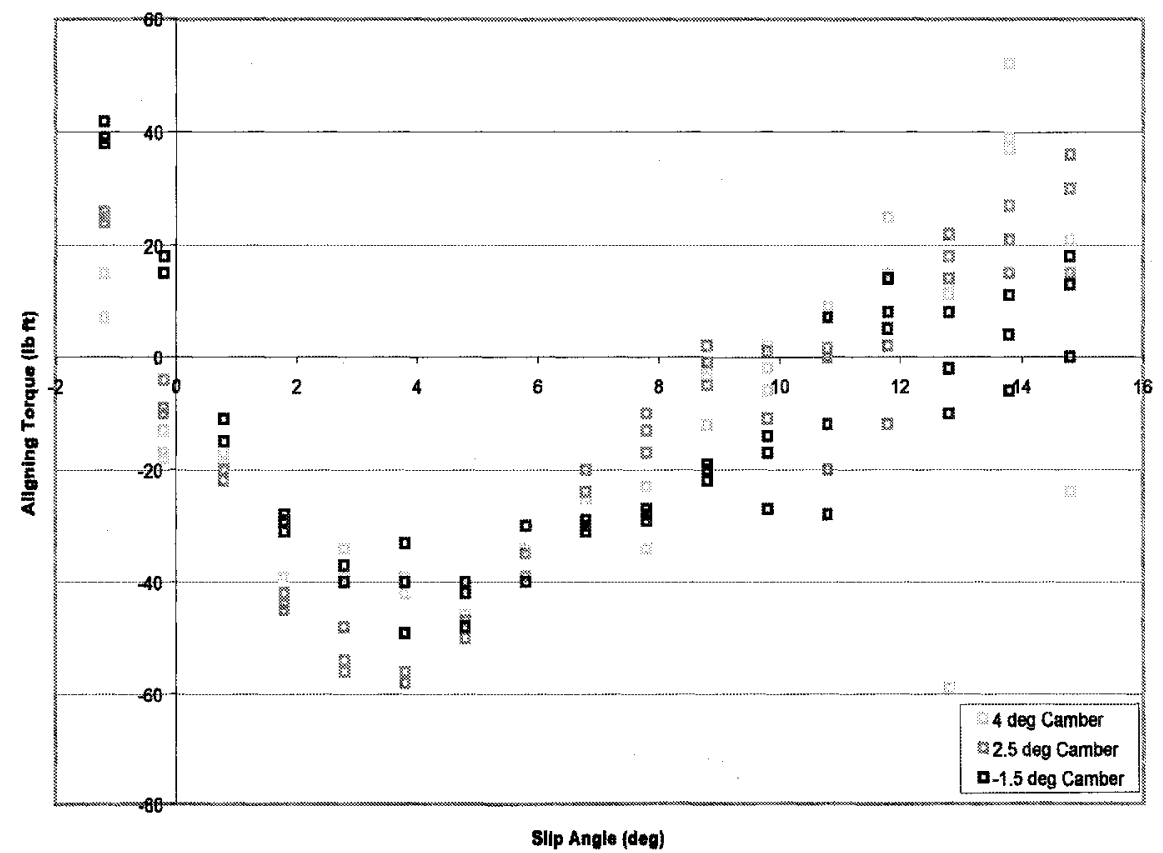

Figure 3.16: Aligning torque versus slip angle empirical test results with $509 \mathrm{lb}$ normal load on an Airtrac 6.00-6 8 ply tire for various camber angles. 


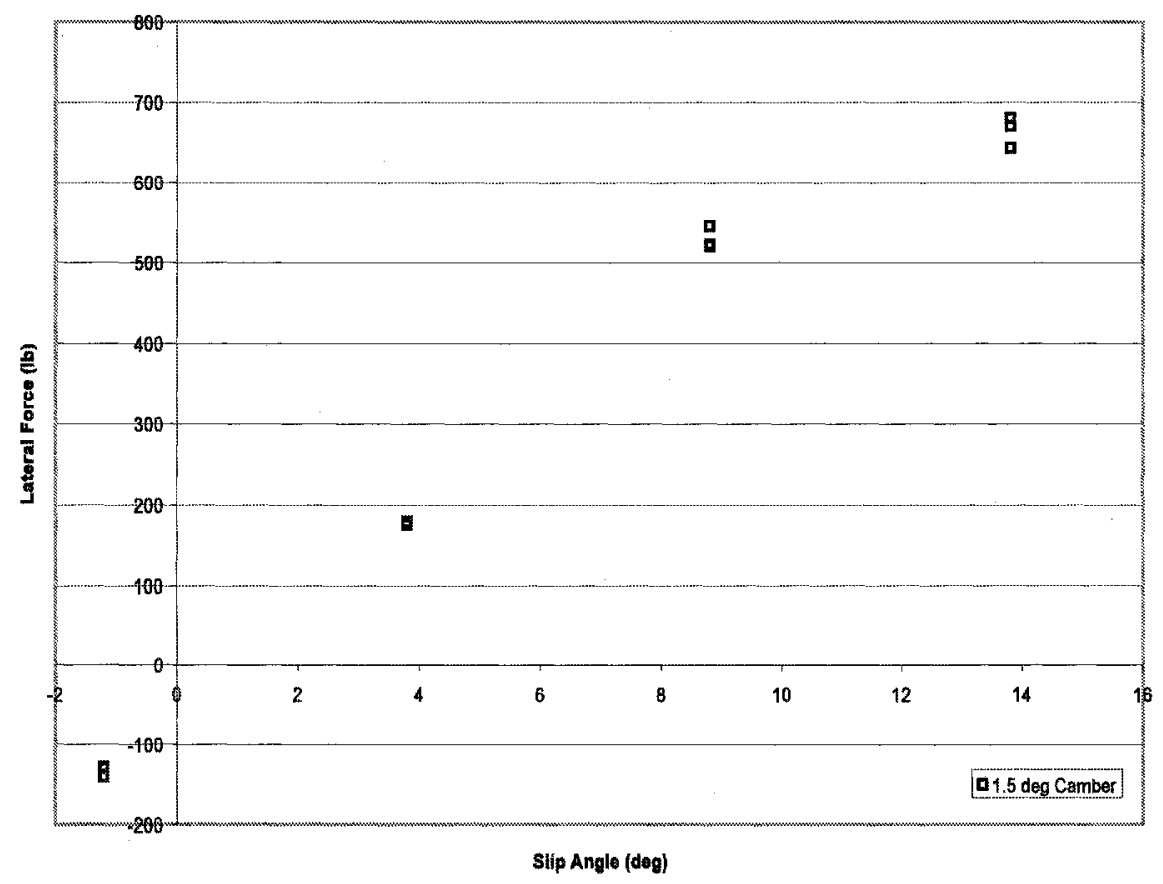

Figure 3.17: Lateral force versus slip angle empirical test results with $591 \mathrm{lb}$ normal load on an Airtrac 6.00-6 8 ply tire angles.

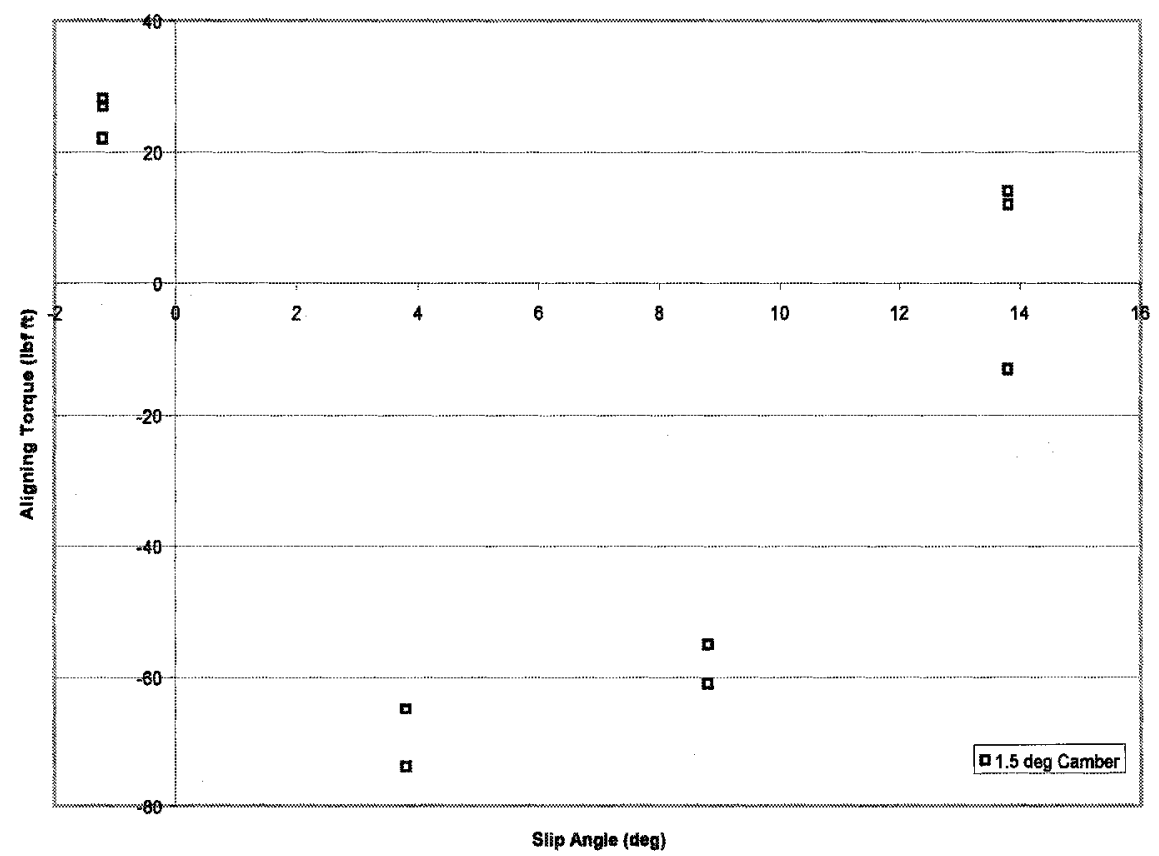

Figure 3.18: Aligning torque versus slip angle empirical test results with $591 \mathrm{lb}$ normal load on an Airtrac 6.00-6 8 ply tire angles. 
the aligning torques. For example, if Figures 3.15 to 3.16 represent the behaviour of a tire on the helicopter where there was a moment arm of $4 \mathrm{ft}$ between the tire and the helicopter's mass centroid, a slip angle of $3^{\circ}$, and a camber angle of $4^{\circ}$, the magnitude of the moment caused by the lateral force would be 15 times that of the aligning torque. The TreadSim computational model will be used to determine an accurate representation of the aligning torque versus slip angle curve for a tire undergoing pure side slip.

\subsubsection{Computational Model Validation and Results}

The TreadSim model was utilized to generate tire data for situations that are not easily tested. To generate a realistic representation of the helicopter tire, appropriate parameters must be chosen for the tire tread's lateral stiffness $c_{p}$ and for the curvature factors $-\epsilon_{\gamma x}$ and $-\epsilon_{\gamma y}$. The lateral stiffness value is easily calculated using Equation 2.31, since $C_{F_{\alpha}}$ is obtained directly from the tire test data. The curvature factors $-\epsilon_{\gamma x}$ and $-\epsilon_{\gamma y}$ are determined by iteratively refining their values until the lateral force and aligning torque versus slip angle graphs match the empirical data. Both factors affect the cornering stiffness and the camber stiffness of the tire. Figures 3.19 and 3.20 show the match between the TreadSim computer simulation results and the empirical date. The values of $-\epsilon_{\gamma x}$ and $-\epsilon_{\gamma y}$ were determined to equal 0.7 and 0.5 respectively. A high level of accuracy was achieved between the empirical data and the simulated data of the lateral force versus slip angle curve.

Since there was an undesirable amount of spread in the aligning torque test results, TreadSim will be used to help model its behaviour. The computational result of the aligning torque versus slip angle curve are in a reasonable position with respect to the empirical data and will be used to create the parameters of the Magic Formula model. 


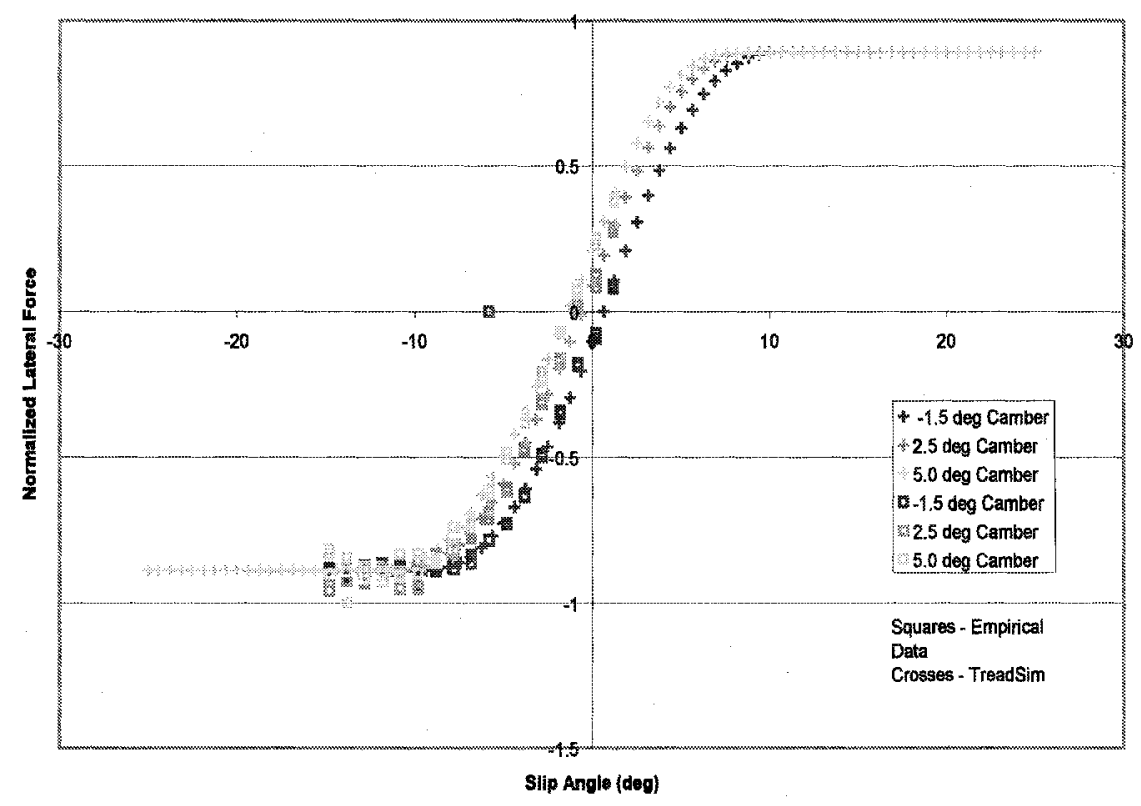

Figure 3.19: Comparison of the TreadSim computer model results to the empirical data for lateral force versus slip angle.

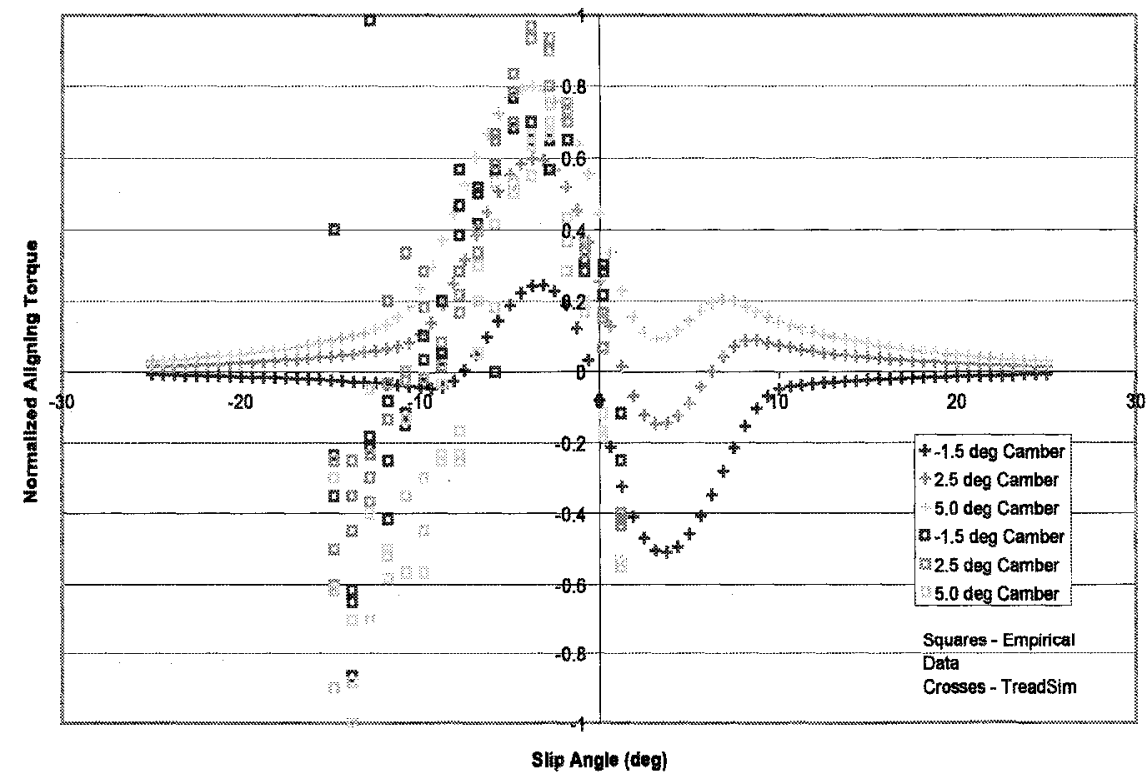

Figure 3.20: Comparison of the TreadSim computer model results to the empirical data for aligning torque versus slip angle. 


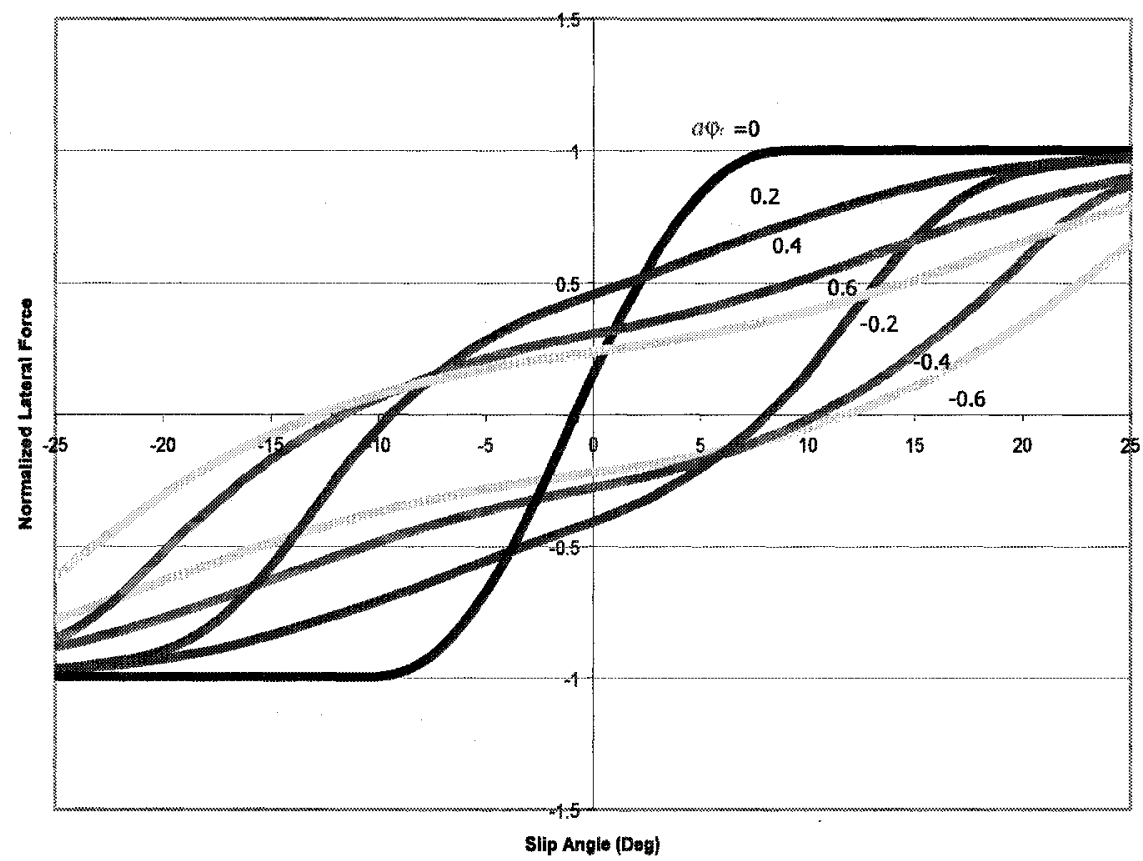

Figure 3.21: Lateral force versus slip angle with turn slip.

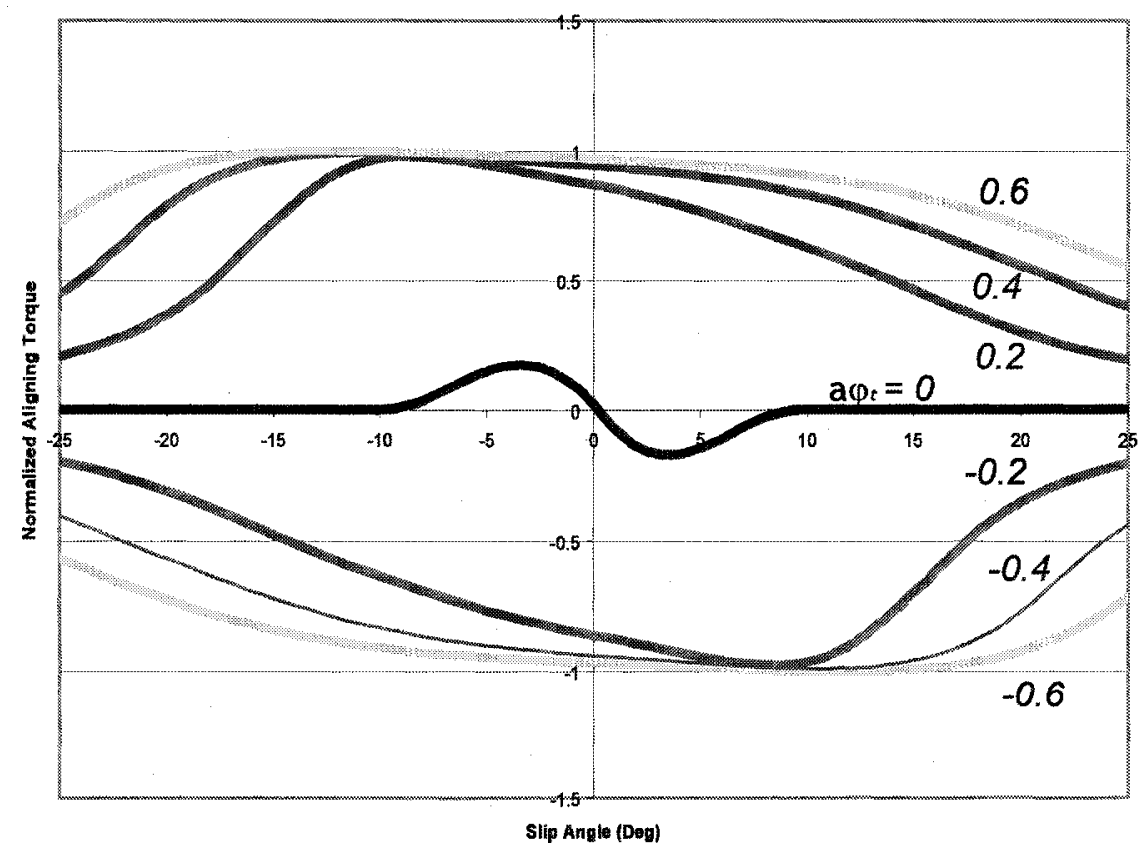

Figure 3.22: Aligning torque versus slip angle with turn slip. 
Once the computer simulation data was properly matched with the empirical data, the simulation was then used to generate the tire force due to turn slip. Turn slip is the, deformation of the contact patch caused by the rotation of the tire about its vertical axis. Figures 3.21 and 3.22 display the results of the computational turn slip model.

\subsubsection{The Magic Formula Tire Model}

The Magic Formula tire model consists of several curve fits that characterize the tire data very well. There is a sigmoid shape curve of the from

$$
Y=D \sin \left[C \operatorname { a r c t a n } \left(B x-E(B x-\arctan (B x))+S_{V}\right.\right.
$$

where

$$
x=X+S_{H}
$$

If $S_{V}$ and $S_{H}$ are equal to 0 then the curve would be perfectly anti-symmetric about the origin. The variables $S_{V}$ and $S_{H}$ allow for the curve to be offset from the origin. The other shape is the bell curve described by

$$
Y=D \cos [C \arctan (B x-E(B x-\arctan (B x)))]
$$

where

$$
x=X+S_{H}
$$

The sigmoid shape curve is used to shape the lateral and longitudinal force versus slip angle curves while the bell shaped curve is used to model the pneumatic trail and residual torque. Both curves are used to shape the characteristics of the turn slip.

The parameters of both curves have physical meanings that help identify their 
values. For the sigmoid curve $B$ is known as the stiffness factor and dictates the slope of the curve near the origin. The D parameter is the peak value factor which is easily identified from the empirical data. The factors $\mathrm{C}$ and $\mathrm{E}$ are known as the shape and curvature factors respectively and can be identified with the help of the relationships

$$
C=1 \pm\left(1-\frac{2}{\pi} \arctan \frac{y_{a}}{D}\right)
$$

and

$$
E=\frac{B x_{m}-\tan \left(\frac{\pi}{2 C}\right)}{B x_{m}-\arctan \left(B x_{m}\right)}
$$

where $y_{a}$ is the minimum value that the curve will sink to after one passes the peak along the $\mathrm{x}$ direction of the curve and $x_{m}$ is the $\mathrm{x}$ coordinate of the peak value.

The factors for the bell shaped curve are determined in a similar fashion. The factor $\mathrm{D}$ is once again the peak value. The factor B must be determined iteratively. The other two factors $\mathrm{C}$ and $\mathrm{E}$ can be solved by the relations

$$
C=\frac{2}{\pi} \arccos \frac{y_{a}}{D}
$$

and

$$
E=\frac{B x_{o}-\tan \left(\frac{\pi}{2 C}\right)}{B x_{o}-\arctan \left(B x_{o}\right)}
$$

where $y_{a}$ is the lowest value of the bell curve and $x_{o}$ is the root of the curve.

The effect of camber on the lateral force curve is modelled by setting $S_{V}$ and $S_{H}$ dependent on the camber. The equations for $S_{V}$ and $S_{H}$ dependent on camber are

$$
S_{V}=C_{M \gamma}\left(F_{z}\right) \gamma+C_{M \alpha}\left(F_{z}\right) S_{H}
$$




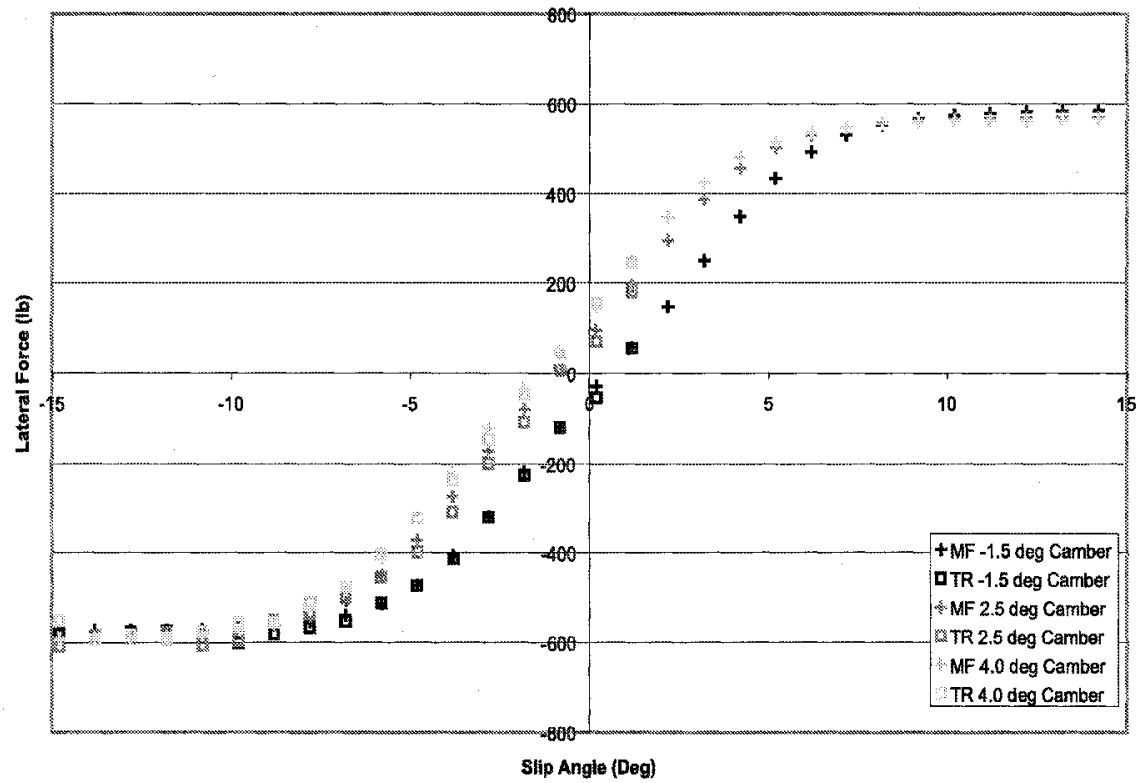

Figure 3.23: Magic Formula model fit of the lateral force versus slip angle to the empirical data.

and

$$
S_{H}=\frac{C_{F \gamma}\left(F_{z}\right)}{C_{F \alpha}\left(F_{z}\right)} \gamma
$$

In Equation 2.58, $M_{z o}^{\prime}\left(\sigma_{e q}^{y}\right)$ is the aligning torque caused by the lateral force and $M_{z r}$ is the residual aligning torque caused by camber. Equation 2.59 is used to control the changes in aligning torque due to camber. Figures 3.23 and 3.24 compare the results of the Magic Formula model fitted with the empirical data. Note that in the legend of the graph MF and TR represents Magic Formula and test results respectively.

The aligning torque versus slip angle curve was fit to match the results of the TreadSim model which is illustrated in Figure 3.25. In the legend of the graph, TS represents the TreadSim results.

The effect of turn slip on the lateral force versus side slip can be categorized the 


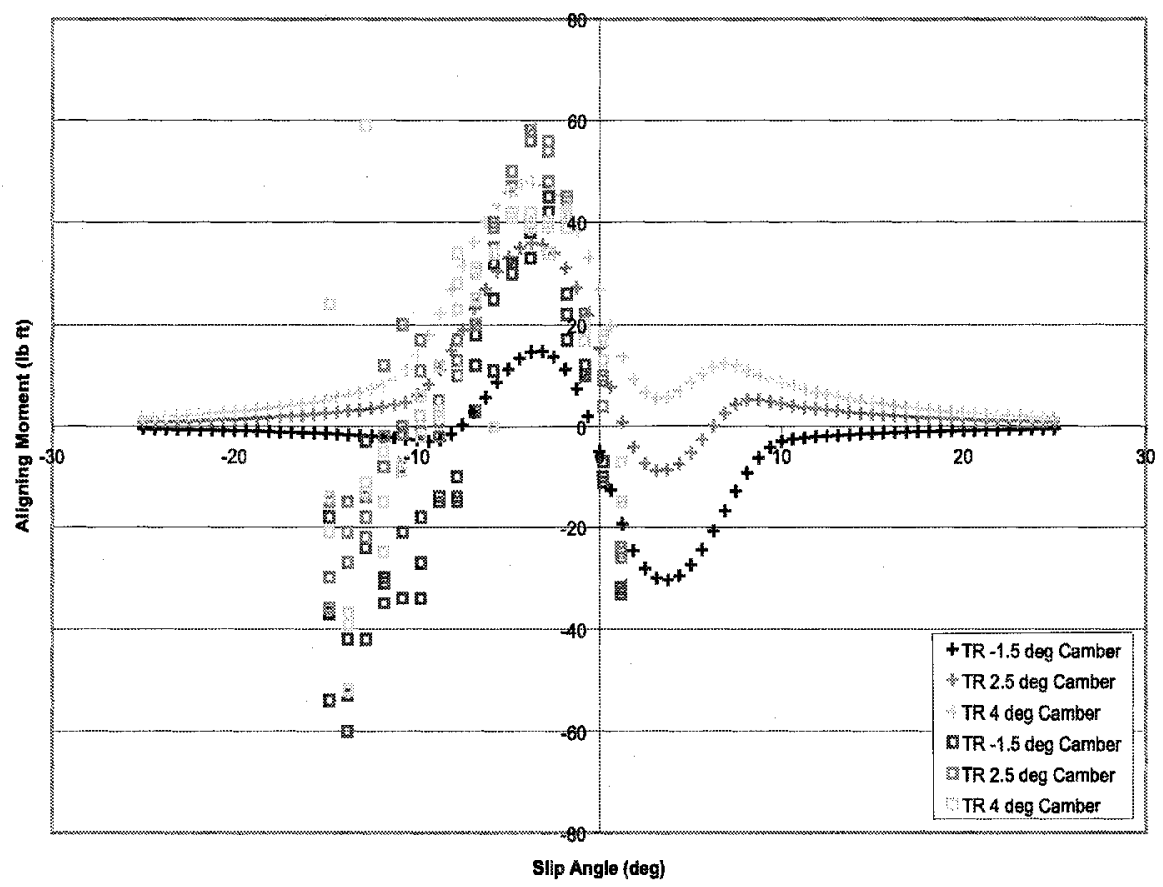

Figure 3.24: Magic Formula model fit of the aligning torque versus slip angle to the empirical data.

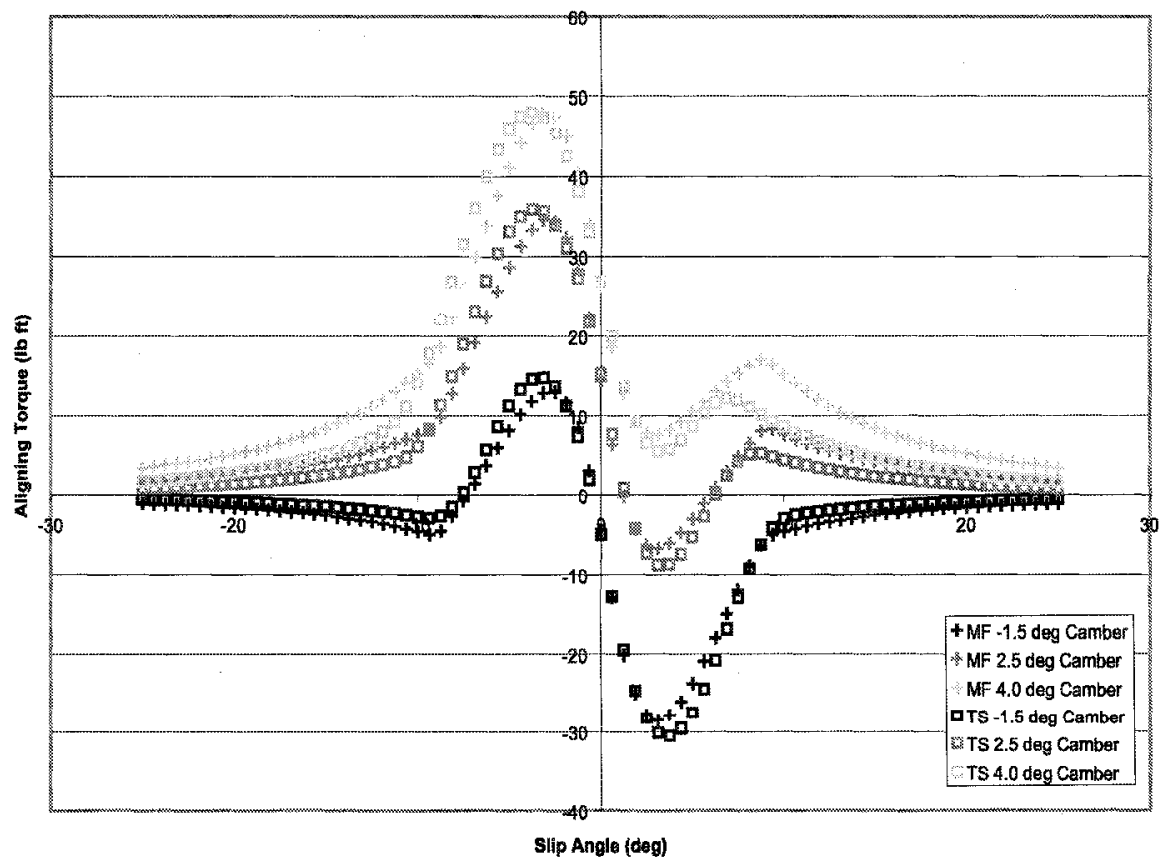

Figure 3.25: Magic Formula model fit of the aligning torque versus slip angle using the TreadSim results. 
effect of three separate categories. The curves will shift in a horizontal direction similar to camber. However, the shift will not be as linear as that of camber. The slope of the graph close to the origin will also decrease with an increase in turn slip. Finally, the location of the peak value of the lateral force will move to a higher value of slip angle as the curve changes from an $\mathrm{S}$ shape to that resembling a line.

To begin the process of fitting the Magic Formula model, the horizontal shift can be determined by graphing the locations that the curves crosses the x-axis at varying degrease of slip. The spacing between these points will begin with a large spread and their distances will decrease as the turn slip increases until the curves of large turn slips all cross the $\mathrm{x}$-axis at a similar point. This behaviour can be fit by the sigmoid shaped curve, Equation 3.1 where $Y$ equals $S_{H_{y \varphi}}$. This relationship is then added to the $S_{H}$ of the overall model. Figure 3.26 is the actual curve fit used for the tire model. Note that the target is the spaces discussed above obtained from a figure similar to Figure 3.21.

The change of slope due to turn slip can be modelled in a similar fashion. The target slope values versus turn slip were graphed and the trend of the data was fit using the bell-shaped curve, Equation 3.3. This relationship, $\zeta_{3}$, is then multiplied by $C_{F \alpha}\left(F_{z}\right)$ in Equation 2.61. Figure 3.27 illustrates the curve fit used in the tire model.

Finally, the peak values are controlled by Equations 2.43 and 2.44 which are

$$
\zeta_{1}=\cos \left(\arctan \left(B_{x \varphi} r_{o} \varphi\right)\right)
$$

where

$$
B_{x \varphi}=c_{x \varphi 1}\left(1+c_{x \varphi 2} F_{z}\right) \cos \left(\arctan \left(c_{x \varphi 3} \kappa\right)\right)
$$

Figure 3.28 shows the final curve product of the Magic Formula curve fit. On the graph the squares represent the Magic Formula model and the addition signs 


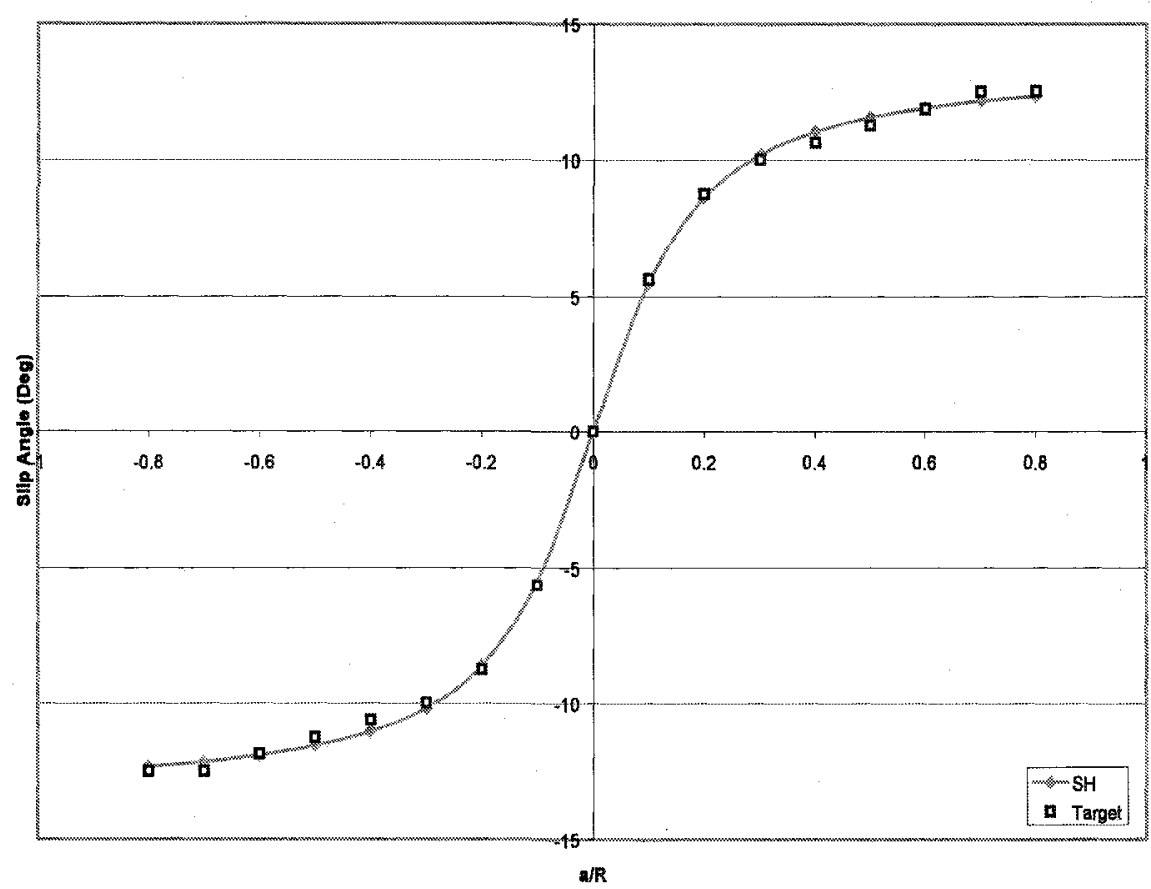

Figure 3.26: Magic Formula model fit of the $S_{H_{y \varphi}}$ spacing caused by turn slip.

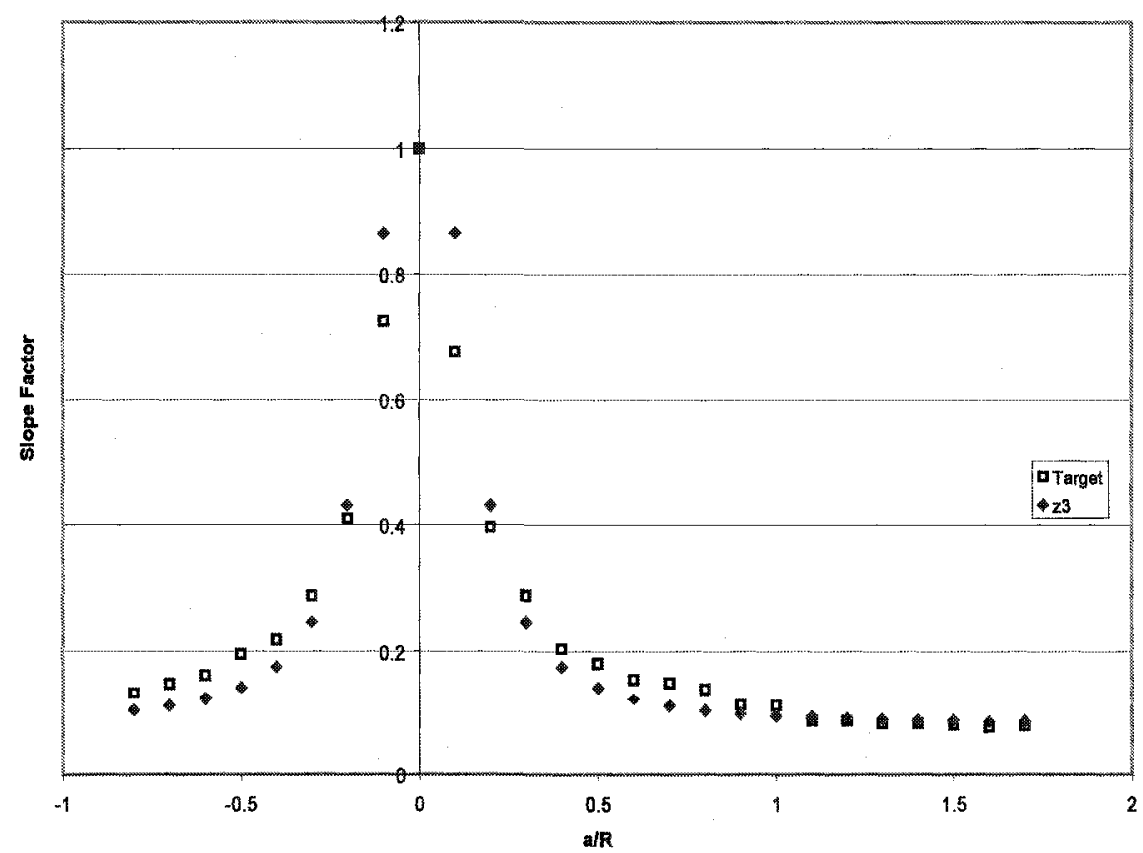

Figure 3.27: Magic Formula model fit of $\zeta_{3}$ caused by turn slip. 


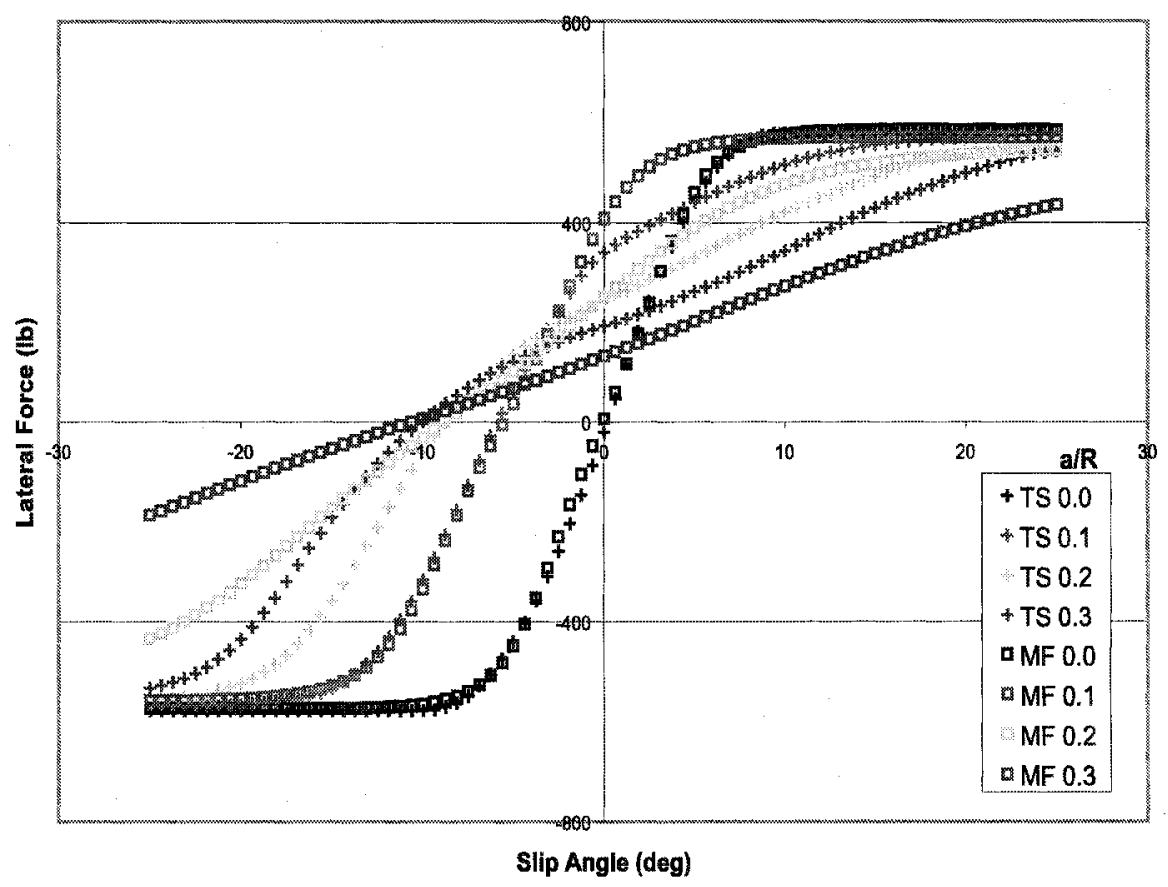

Figure 3.28: Magic Formula fit of the lateral force versus slip angle with turn slip.

represent the data collected from the TreadSim model.

Turn slip will cause the pneumatic trail to decrease and the residual torque to increase. The curves are fitted using the relations described in Section 2.3.2. The overriding effect of the aligning torque is the increase of residual torque. The peak values of the aligning torque are mainly from the residual torque. Therefore, a graph of the peak values of aligning torque versus turn slip can be created to identify a relationship for $D_{r \varphi}$ using Equation 2.49. Figure 3.29 demonstrates this concept. Figure 3.30 demonstrates the Magic Formula fit to the data collected from the TreadSim model. 


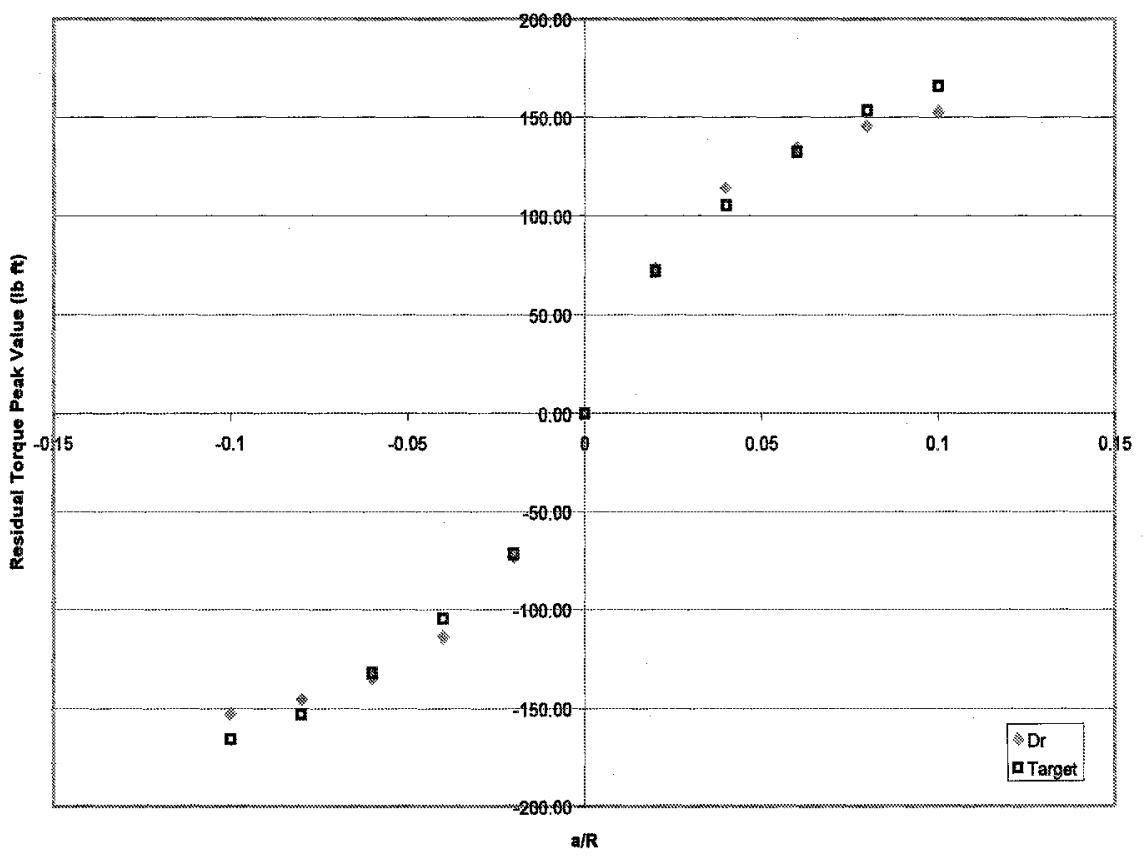

Figure 3.29: Residual torque peak value versus turn slip.

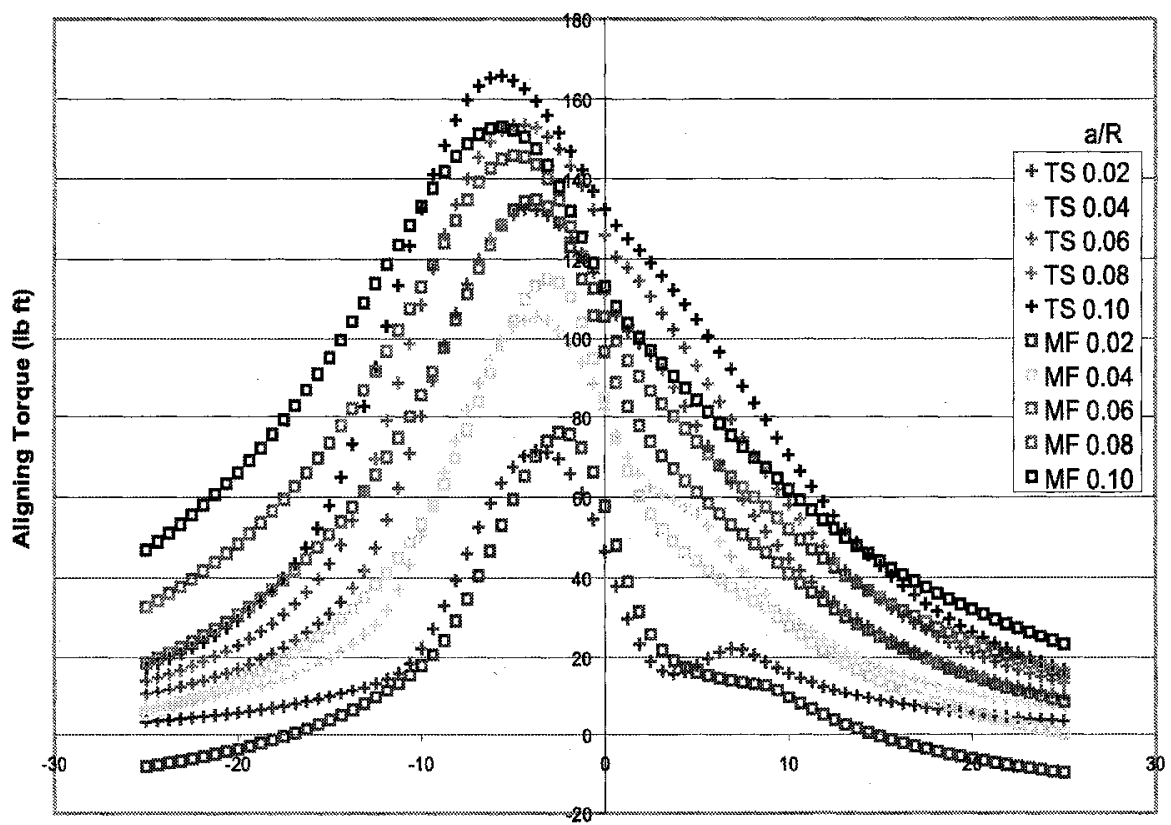

Slip Angle (deg)

Figure 3.30: Aligning torque versus slip angle with turn slip. 


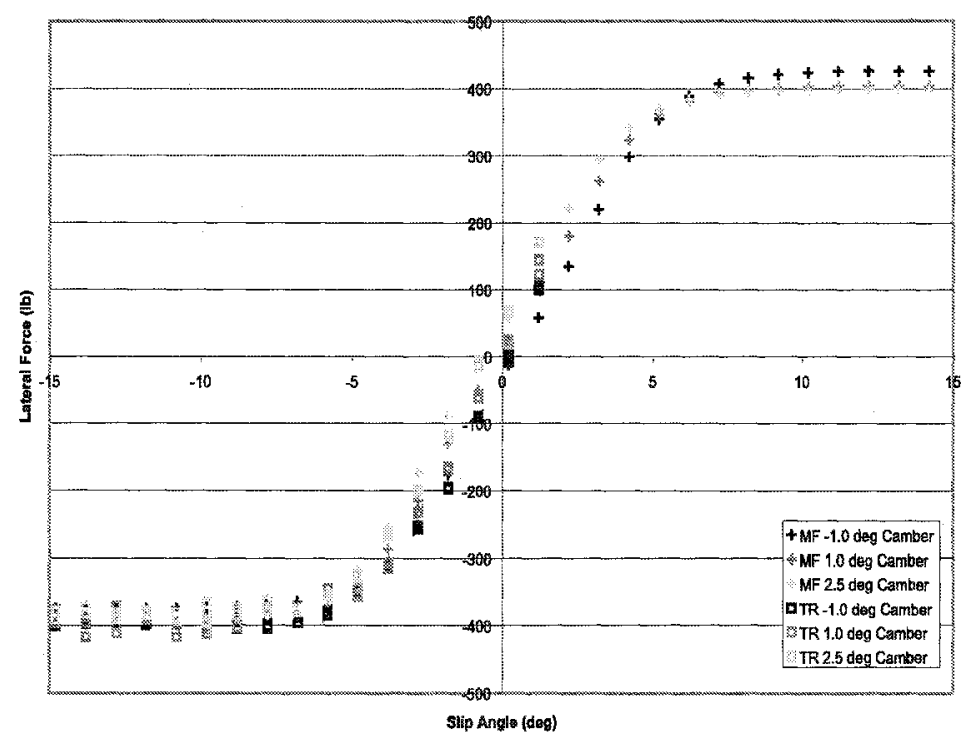

Figure 3.31: Comparison of the lateral force versus slip angle curve generated by the Similarity Method to the empirical data collected with the normal load set at $351 \mathrm{lb}$.

\subsubsection{The Similarity Method Tire Model}

The Similarity Method Tire Model takes the Magic Formula curve fits and adjusts them for changes in normal loading or frictional coefficients. The above Magic Formula curve fits were applied to the 509lb data set. Figures 3.31 through 3.34 demonstrate the accuracy of the similarity method model by comparing the generated curves due to normal load change to the empirical data collected. 


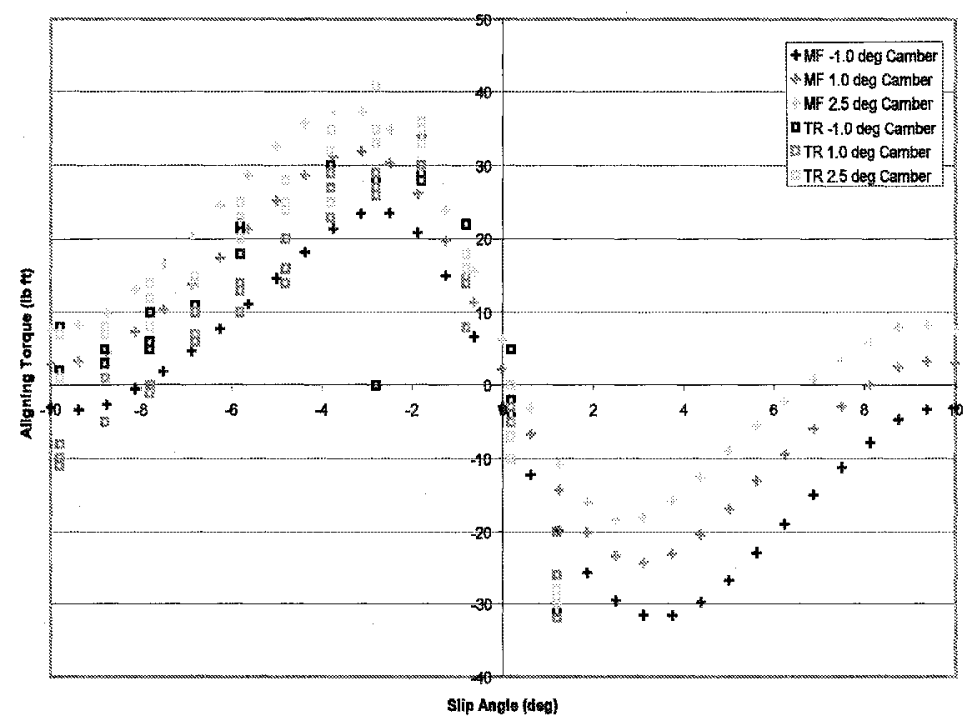

Figure 3.32: Comparison of the aligning torque versus slip angle curve generated by the Similarity Method to the empirical data collected with the normal load set at $351 \mathrm{lb}$.

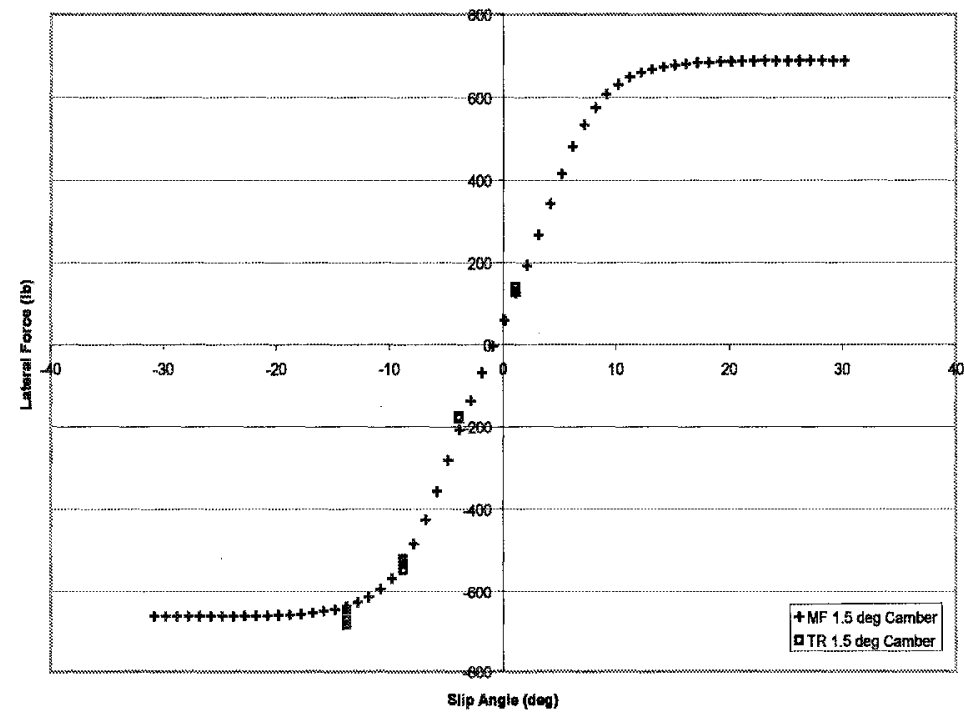

Figure 3.33: Comparison of the lateral force versus slip angle curve generated by the Similarity Method to the empirical data collected with the normal load set at $592 \mathrm{lb}$. 


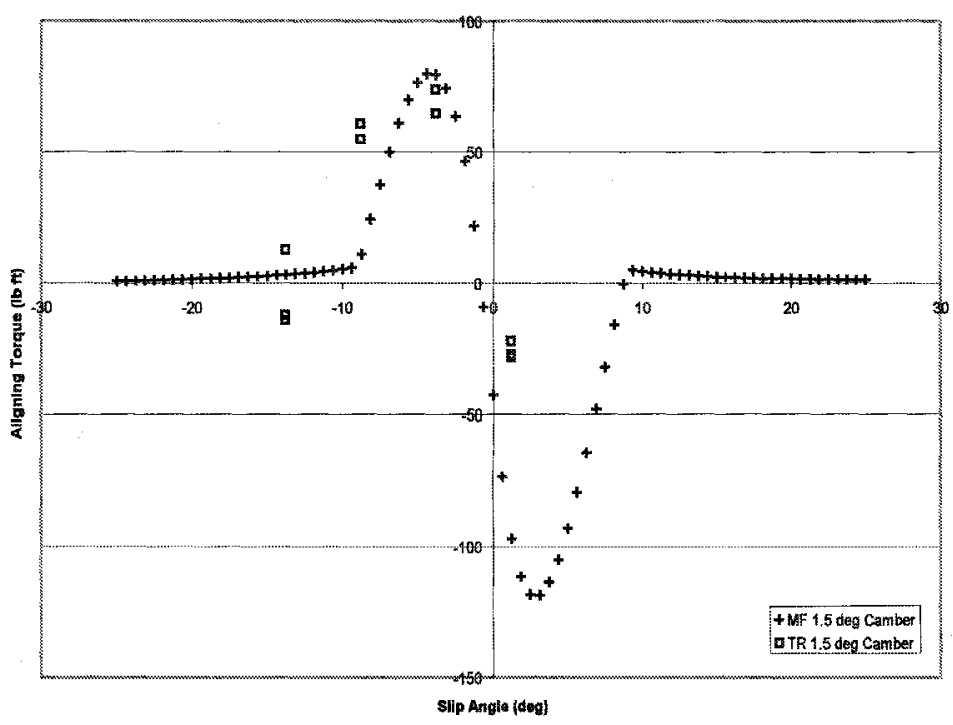

Figure 3.34: Comparison of the aligning torque versus slip angle curve generated by the Similarity Method to the empirical data collected with the normal load set at $592 \mathrm{lb}$. 


\section{Chapter 4}

\section{Real Time Tire Computational}

\section{Simulation}

The steady state tire model developed in the previous chapter was used to improve the planer helicopter shipboard manoeuvring simulation called HeliMan [1]. The steady state tire model combined with the single point transient tire model solves the fully nonlinear tire force problem, and is applicable for use in a full three-dimensional environment. This chapter will explain and demonstrate the real time tire model created to improve the HeliMan software.

\subsection{The HeliMan Computational Simulation}

The HeliMan computational simulation software is a program that models the dynamics of a shipboard helicopter under the control of the ASIST securing and traversing system. The simulation is applicable for studying the dynamic behaviour of the system, training future operators, and providing a model for future work on autonomous control of the system. For a complete review of the simulation development refer to 


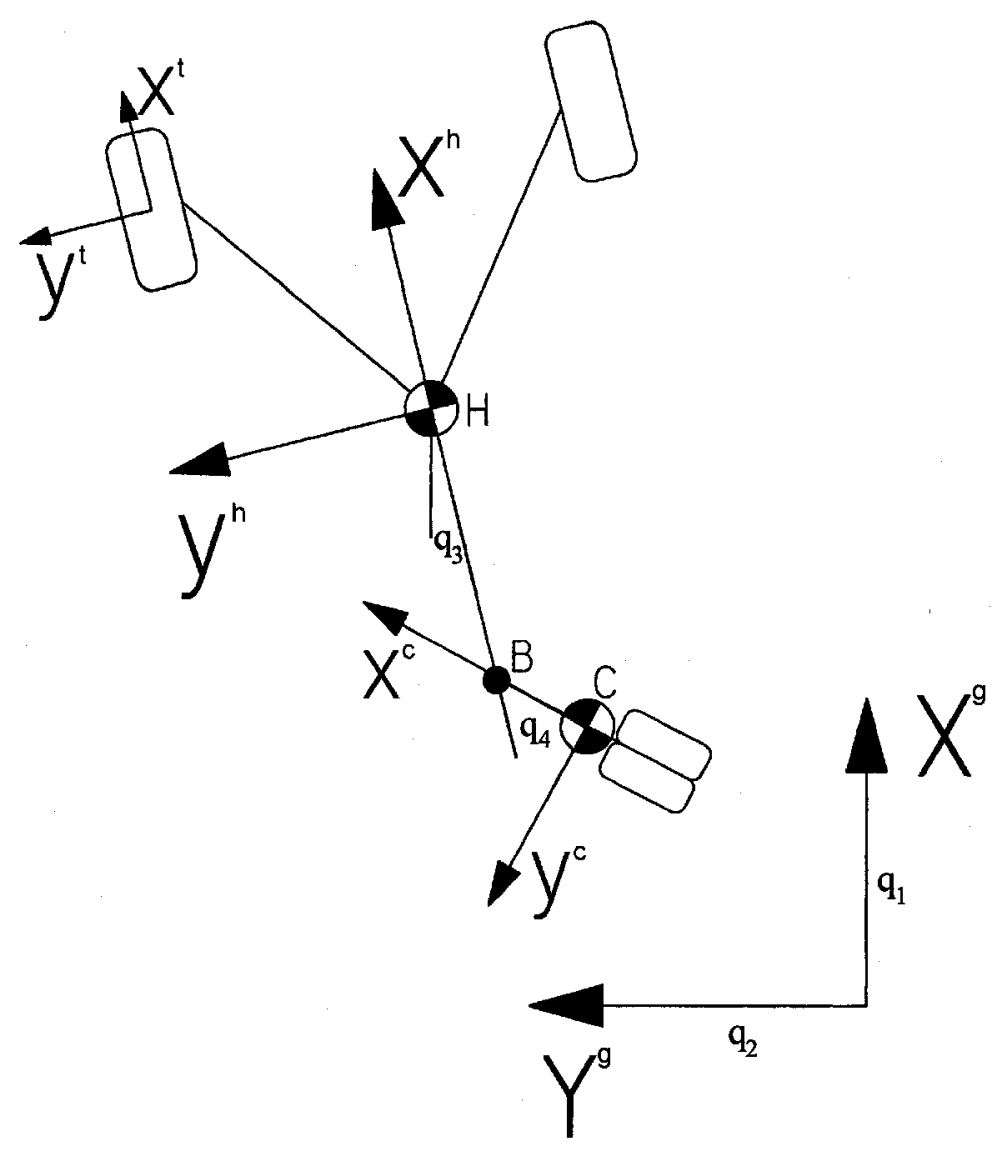

Figure 4.1: Schematic of the dynamic helicopter model.

Linn [1].

The dynamics of the planer helicopter were set up using four degrees of freedom as shown in Figure 4.1. The four generalized coordinates $q_{i}$ are: the linear $\mathrm{x}$ and $\mathrm{y}$ components of the position of the helicopter centre of mass, the orientation of the helicopter, and the orientation of the castor wheel relative to the helicopter body. The figure also illustrates the helicopter $\mathrm{h}$, ground $\mathrm{g}$, and tire $\mathrm{t}$, coordinate systems used in the model development.

Figure 4.2 shows a free body diagram of the helicopter and castor wheel assembly. The reaction forces of the castor joint were required to properly model the frictional torque of the joint at location B. $\vec{F}_{d x}$ and $\vec{F}_{d y}, \vec{F}_{p}$, and $\vec{F}_{t}$, represent the aerodynamic drag force components, probe force, and tire forces acting on the helicopter respec- 

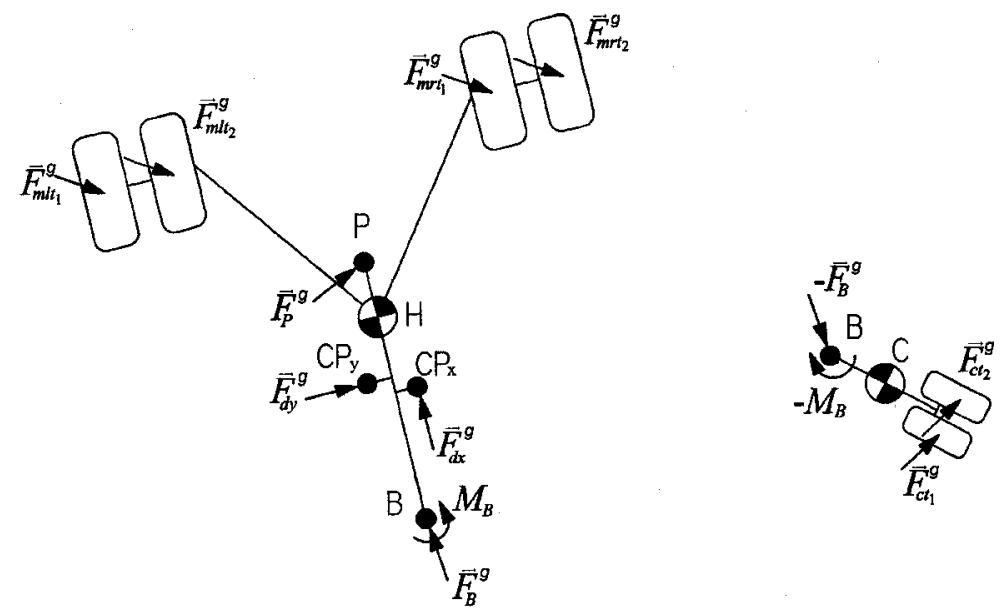

Figure 4.2: Free-body diagram of the main helicopter body and castorable wheel assembly.

tively. The equations of motion as well as the castor joint, probe force, and control models are developed in detail by Linn [1]. The numerical integration algorithm used to solve the differential equations governing the helicopter's dynamics wis a fourth order fixed time step Runge-Kutta method.

\subsubsection{Tire Models Comparison}

Special attention must be given to the forces generated by the tires of the traversing helicopter simulation when compared to a conventional motor vehicle simulation. Simulation of the tire forces on a car driving on a road is an easier task since the tires will only experience small amounts of lateral slip. For small degrees of lateral slip, the mathematical equations governing the forces generated by the tires, Equations 2.71 and 2.72, are linear in nature. Both the cornering stiffness and the relaxation length can be treated as constants. In the case of the helicopter traversing, the helicopter probe can be pushed by the ASIST handling system in any direction which may generate large degrees of lateral slip. It is important that the tire simulation model incorporate all the nonlinear properties for the case of the traversing helicopter. The 
nonlinear equations, Equations 2.76 and 2.77, must be used. These equations require a complete steady state tire model in order to solve the instantaneous relaxation length represented by Equation 2.75 .

The model used by Linn [1] was developed by Smiley and Horne [17]. The tire model was developed from empirical testing of several classes of standard aviation tires. However, this model was published in the mid $20^{\text {th }}$ century when the main design of tires consisted of a bias ply lay up as opposed to the current almost exclusive use of radial ply tires for aircraft applications.

Comparisons can be made between the Smiley and Horne tire model and the single transient tire model presented by Pacejka [6]. The older model uses an equation analogous to Equation 2.77. The solution to Equation 2.77 is:

$$
v_{\alpha_{i}}=\frac{-\sigma_{\alpha}^{*}}{V_{x}} V_{s y}+e^{\frac{V_{x}}{\sigma_{\alpha}^{*}}\left(t_{i-1}-t_{i}\right)}\left(v_{\alpha_{i-1}}+\frac{\sigma_{\alpha}^{*}}{V_{x}} V_{s y}\right)
$$

where $t$ represents time and subscript $i$ represents the current time step.

However, the steady state tire deformation and the relaxation length $\sigma_{\alpha}^{*}$ are determined by empirical relations derived from experimental tire test results. The empirical relations for the relaxation length are a function of the ratio of tire pressure $q_{z}$ to nominal tire pressure $q_{z o}$ and are:

$$
\begin{array}{llrl}
\sigma_{\alpha}=\frac{11 d_{z}}{2 r_{t}}\left(2.8-\frac{0.8 q_{z}}{q_{z o}}\right) 2 b & \text { for } & & \frac{d_{z}}{2 r_{t}} \leq 0.053 \\
\sigma_{\alpha}=\left(\frac{64 d_{z}}{2 r_{t}}-500\left(\frac{d_{z}}{2 r_{t}}\right)^{2}-1.4045\right)\left(2.8-\frac{0.8 q_{z}}{q_{z o}}\right) 2 b & \text { for } & 0.053>\frac{d_{z}}{2 r_{t}}<0.068 \\
\sigma_{\alpha}=\left(0.9075-\frac{4 d_{z}}{2 r_{t}}\right)\left(2.8-\frac{0.8 q_{z}}{q_{z o}}\right) 2 b & \text { for } & & \frac{d_{z}}{2 r_{t}} \geq 0.068
\end{array}
$$


where $d_{z}$ and $r_{t}$ represent the tire's vertical deformation and radius respectively.

The relaxation length for Pacejka's transient tire model is determined by the instantaneous cornering stiffness (the instantaneous slope of the lateral force versus slip angle curve) divided by the lateral tire stiffness. The lateral force versus slip angle curve is a function of normal force (which determines tire pressure). Therefore, the relaxation lengths of both models are functions of normal force. The difference between the two models is that Smiley and Horne's relaxation length is a constant while the normal force is constant. Pacejka's relaxation length is a function of the transient slip angle. The relaxation length will only be constant in the linear portion of the mathematical equations when the transient slip angle is small. Also, Smiley and Horne's relaxation length has three generic settings based on the value of $\frac{d_{z}}{2 r_{t}}$. Pacejka's relaxation length is adapted to any tire by the use of the Steady State Magic Formula model.

The second fundamental difference between the tire models is the determination of the steady state tire deformation for a given slip angle. In Equation 4.1, $\frac{-\sigma_{x}^{*}}{V_{x}} V_{s y}$ is the steady state lateral deformation of the tire for the given transient value of slip angle $\alpha^{\prime}$. In Pacejka's model, the steady state force is determined through the use of the Steady State Magic Formula model. Smiley and Horne use a third order polynomial that is a function of slip angle to determine the steady state force. Therefore, the older model's steady state lateral force versus slip angle curve has one specific shape for a given normal force.

The design of aircraft tires (and tires in general) have improved since then and, as indicated previously, radial tires are now more prevalent. Recently, a study of several standard aviation tires was conducted by Daugherty [22] at the Langley Research Center. Daughterty conducted tire tests on three large aviation tires used on Boeing $777 \mathrm{~s}$ and on Boeing $737 \mathrm{~s}$. The results show the effects of inflation pressure, normal 


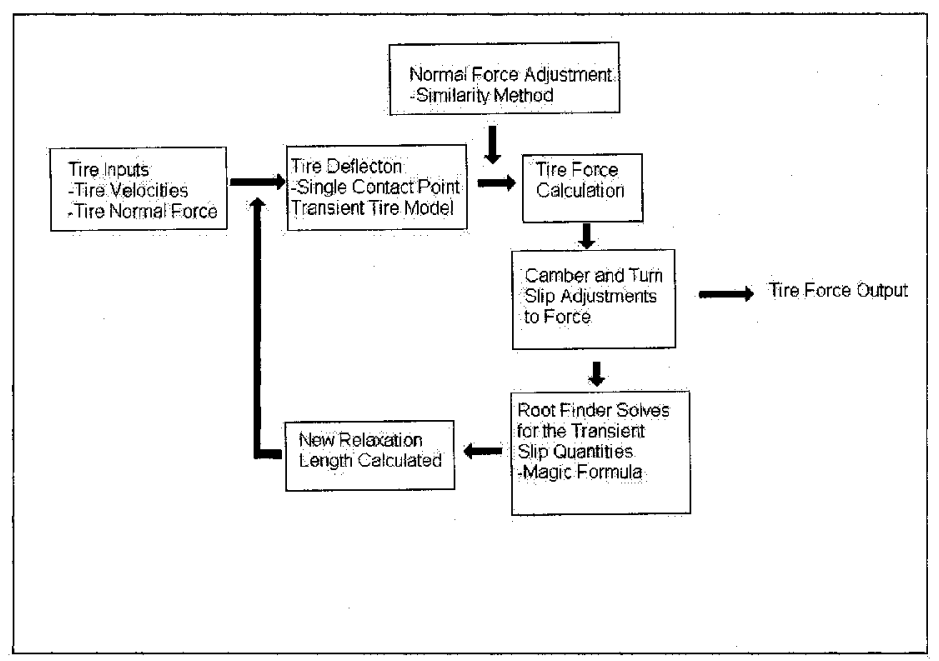

Figure 4.3: Block diagram of the computational tire model.

force, and slip angle on tire behaviour. The tires used for this study were larger, experienced a greater normal force, and were composed of a higher ply count than those used in the HeliMan simulation.

Pacejka's tire model allows for more flexibility with the resulting tire behaviour than that of Smiley and Horne. It also allows for the addition of forces and moments caused by camber and turn slip. Adjusting the values of the Steady State Magic Formula allows Pacejka's tire model to be adapted to any tire simulation application.

\subsubsection{New Tire Model Application}

The new tire model calculates the forces by following the steps shown in Figure 4.3.

First, the quantities of longitudinal slip and side slip are determined by the contact patch slip velocity components. These inputs, as well as initial tire deflections, are used to determine the tire's deflections using the single contact point transient tire model introduced in Section 2.5.1. The tire forces are determined next by multiplying the tire deflections by their respective tire stiffnesses. If there is a normal force change 
or a frictional coefficient change that differs from the base variables, adjustments are made by the use of the Similarity Method presented in Section 2.4.1. The amount of transient slip is determined by finding the transient slip quantity that corresponds to the tire force through the use of a root finding technique on the tire behaviour curves, modelled using the Magic Formula equations. The transient slip values are used to adjust the relaxation length for the next time step. The relaxation length will control the length of growth and decay of the transient force. This process repeats itself at each time step of the program.

\subsection{Validation of the Real Time Tire Simulation}

During the development of the helicopter ship flight deck traversing real time computational simulation known as HeliMan, test data was collected from a full-scale experimental apparatus at Indal Technologies Inc. The test apparatus consisted of a Dead Load Test Vehicle (DLTV) that was configured to emulate a Seahawk helicopter controlled by the ASIST securing and traversing mechanism, shown in Figure 4.4. The Seahawk helicopter has two front tires and a dual-wheel castor tail wheel assembly.

A wide variety of manoeuvres were performed for comparison purposes. The test apparatus was equipped with instruments that measured the lateral deformation of the front wheels, the reaction force on the probe securing the helicopter, and the orientation of the castor wheel. The experiment was also stopped intermittently to allow hand measurements of the helicopter's orientation relative to the ground. The position of the ASIST probe was recorded and used as input for the HeliMan simulation comparisons. There were seven manoeuvres performed by the DLTV experiment for comparison purposes that include: 


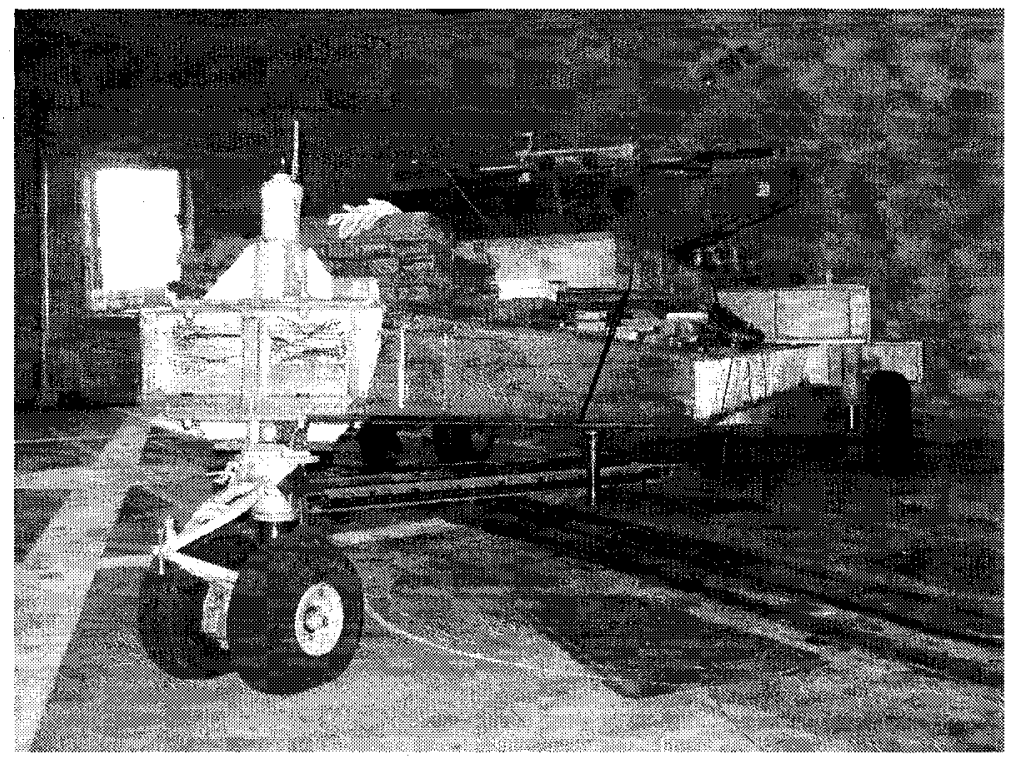

Figure 4.4: Dead load test vehicle emulating a Seahawk helicopter.

1 a lateral stiffness test;

2 traversing while yawed;

3 yawing to 90 degrees;

4 yawing back to 0 degrees with free float from 90 degrees;

5 DLTV 180 degrees castor rotation;

6 DLTV traversing to relax tire deflexion; and

7 a typical complex manoeuvre.

Linn [1] used cases one through six to validate the older tire model. The new tire model was validated in a similar fashion with several exceptions. Cases two and six are redundant tests of a simple manoeuvre that is used to observe the transient behaviour of the tire. Case five is only a slightly more complicated version of cases three and four. Case seven was compared since it involved a lengthy straightening procedure that demonstrated a complex manoeuvre and gives a better overall comparison of the tire model's performance. The the test cases used in the simulation validation of the new tire model are: 
1 lateral stiffness;

3 yawed to 90 degrees;

4 yawing back to 0 degrees with free float from 90 degrees;

7 a typical complex manoeuvre.

For each test case, the reaction forces acting on the probe, the lateral tire deflection of the front tires, and when appropriate, the orientation of the castor wheel will be compared with the experimental data and the old tire simulation model. Figure 4.5 , depicts the computational simulation program as seen on the computer screen and illustrates the orientation of the ship deck. The complexity of the manoeuvre increases with each test case.

\section{Case 1: Lateral Stiffness}

The first test case is used to determine the proper stiffness of the front tires. The tires traverse mainly in their lateral direction causing an almost pure lateral slip condition. This case allows the force to be compared with the tire deflection to determine the lateral stiffness of the tire's.

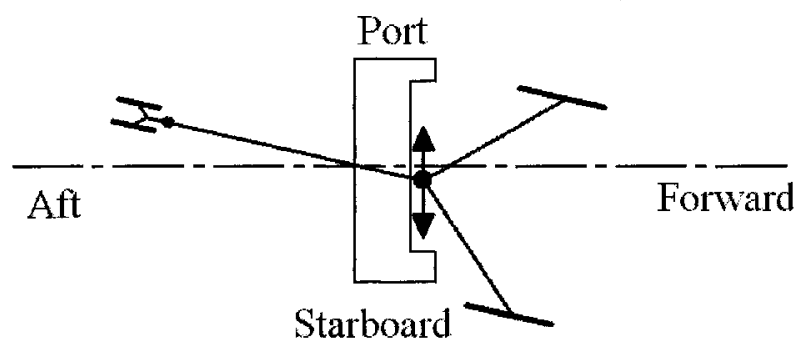

Figure 4.5: A representation of the computational simulation.

In order to test for the lateral stiffness of the tires, the dead load test vehicle was traversed back and forth in the lateral direction while the helicopter was positioned with a small orientation angle to starboard. Figure 4.5 gives a visual representation 
of the helicopter's position relative to the fight deck. The castor wheel was locked in position, disallowing any rotation. Figure 4.6 graphs the y-position of RSD claw for both the experiment and the simulation. The simulation's input position is closely matched by the actual probe displacement in the experiment with only a slight difference when traversing in the port/straboard direction.

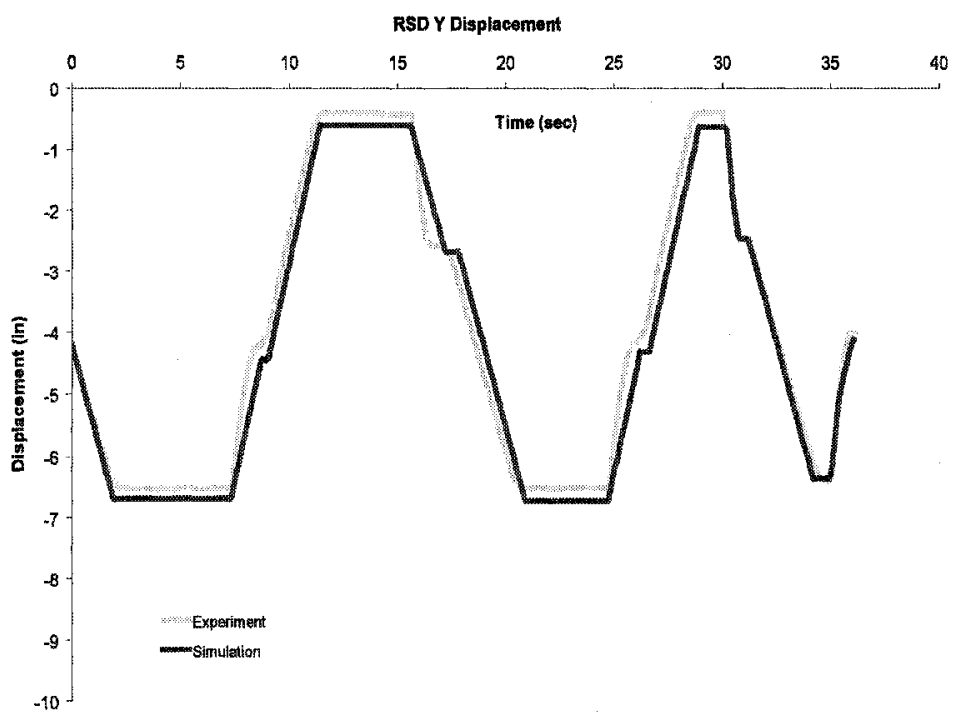

Figure 4.6: Comparison of the RSD claw y-position for Case 1.

Figure 4.6 shows the force on the probe acting in the $y$-direction relative to the helicopter with the positive direction pointing toward the port side. Both the old and new tire models demonstrate an accurate prediction of the force. Figure 4.8 shows the probe force in the $\mathrm{x}$-direction relative to the helicopter. The simulation slightly underestimates the force recorded in the experiment. However, the new tire simulation's results are slightly better with no force decay present during the stationary periods of the experiment.

The tire defection for the right and left tires are presented in Figures 4.9 and 4.10 respectively. Differences exist in the tire deflection when the helicopter is resting starboard. The old tire model overestimates the deflection while the new tire model 


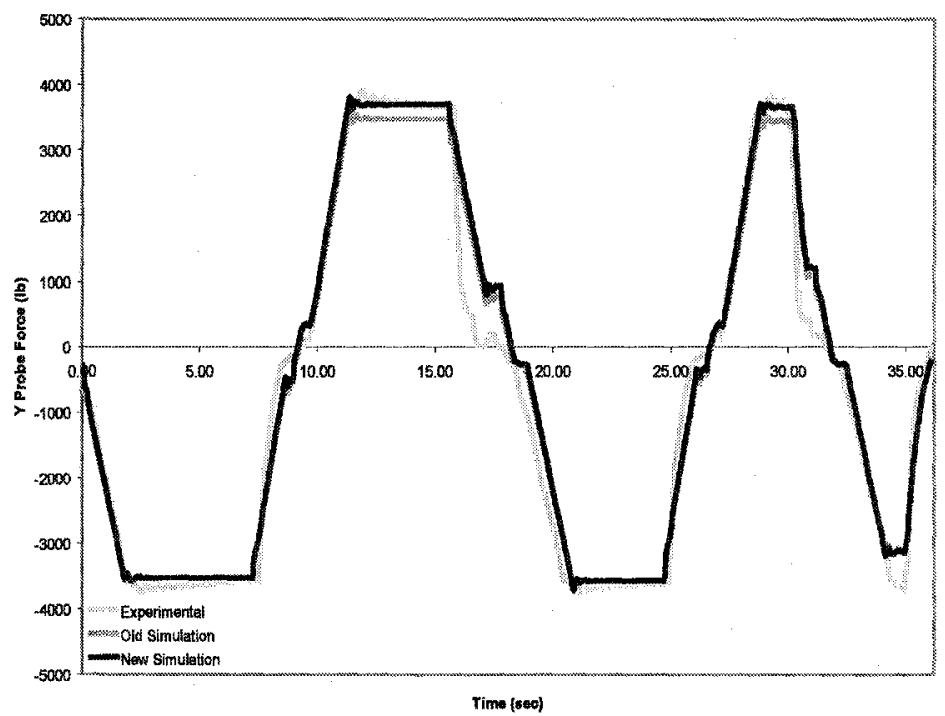

Figure 4.7: Comparison of the force acting on the probe in the DLTV's $\mathrm{y}$-direction for Case 1.

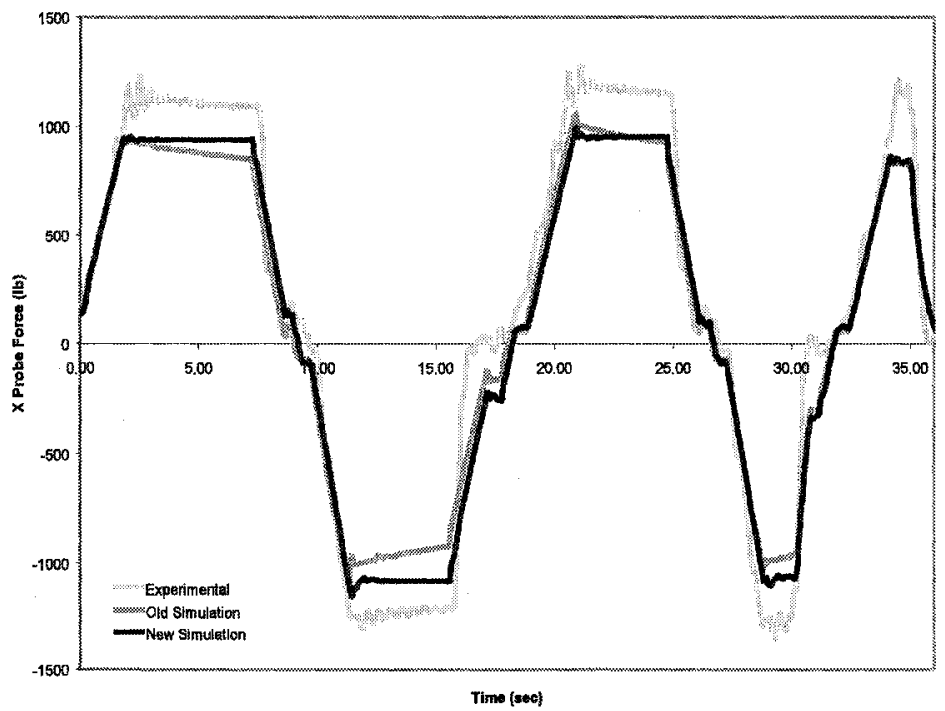

Figure 4.8: Comparison of the force acting on the probe in the DLTV's $\mathrm{x}$-direction for Case 1 . 


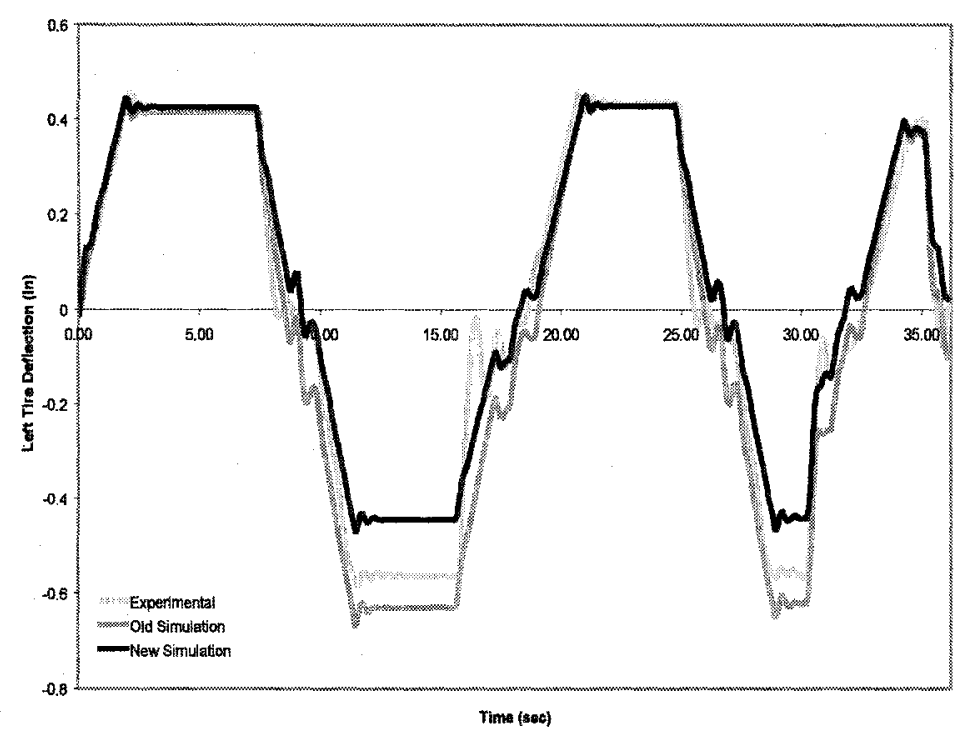

Figure 4.9: Comparison of the lateral deflection of the right tire for Case 1.

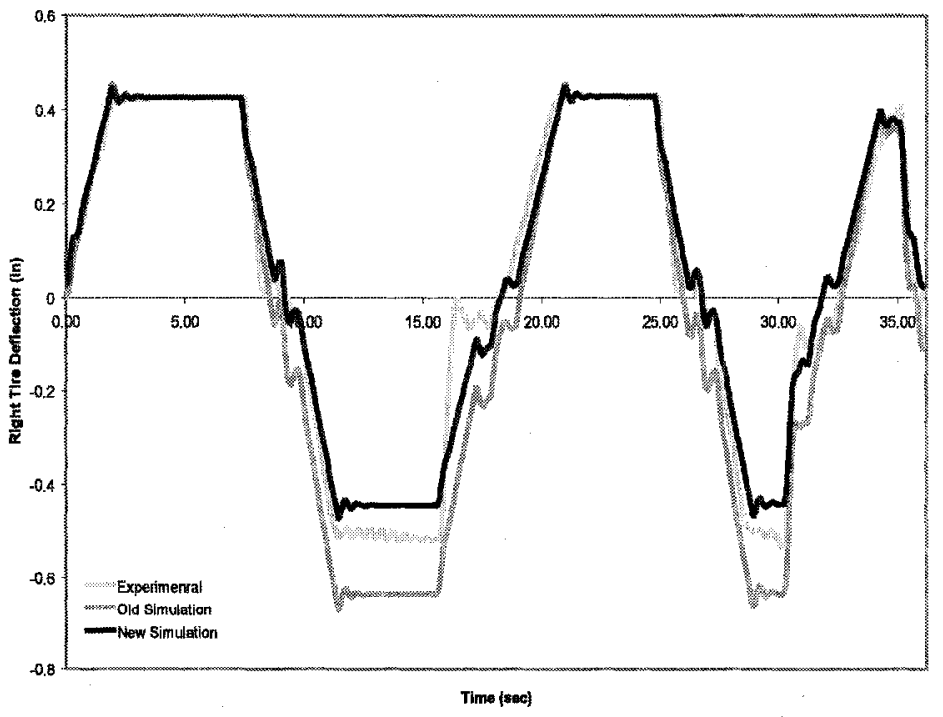

Figure 4.10: Comparison of the lateral deflection of the left tire for Case 1. 
underestimates it. This is peculiar since a linear relationship exists between the tire force and displacement. Since the tire had the right deflection during the helicopter's travel in the port direction the same should be the case when travelling in the starboard direction. Also, the force generated in the y-direction on the probe is correct. This suggests that there may have been discrepancies in data collection when recording the displacement of the tires. The difference in tire deflection between the right and left tires in the starboard position also supports the scepticism.

\section{Case 3: Yawed to 90 Degrees}

For the second test case, the DLTV performed a manoeuvre that started with the DLTV's longitudinal axis almost parallel with the longitudinal axis of the deck. The DLTV is orientated $9^{\circ}$ towards port. The RSD pulls the probe in the deck's longitudinal direction, making the DLTV yaw with the front of the DLTV orientation changing until the front is facing $90^{\circ}$ to port. Figure 4.11 illustrates the starting position of the helicopter.

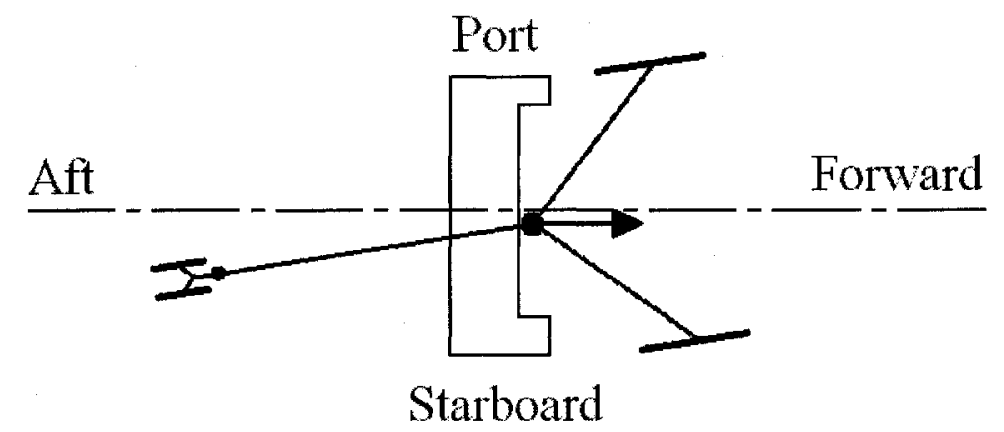

Figure 4.11: Initial position of DLTV and direction RSD is moved for Case 2.

Figures 4.12 and 4.13 compare the position of the probe with the experiment and simulation, showing consistant displacements. The purpose of the manoeuvre is to test the simulated helicopter model, by making it rotate $90^{\circ}$. To ensure that the simulation is implementing the correct dynamics, it is important that the freely 
rotating castor wheel have the correct orientation. Figure 4.14 compares the simulated castor wheel orientation with the actual orientation of the DLTV castor assembly. Both the new and old tire models create results that correlate well with the actual experiment. During the experiment, the DLTV was stopped at various time intervals throughout the manoeuvre so that manual measurements of the helicopter could be made. Both tire models show the tires rotating during these phases. A comparison of the helicopter's orientation relative to the ground is shown in Figure 4.15. The new and old tire models produce nearly identical results.

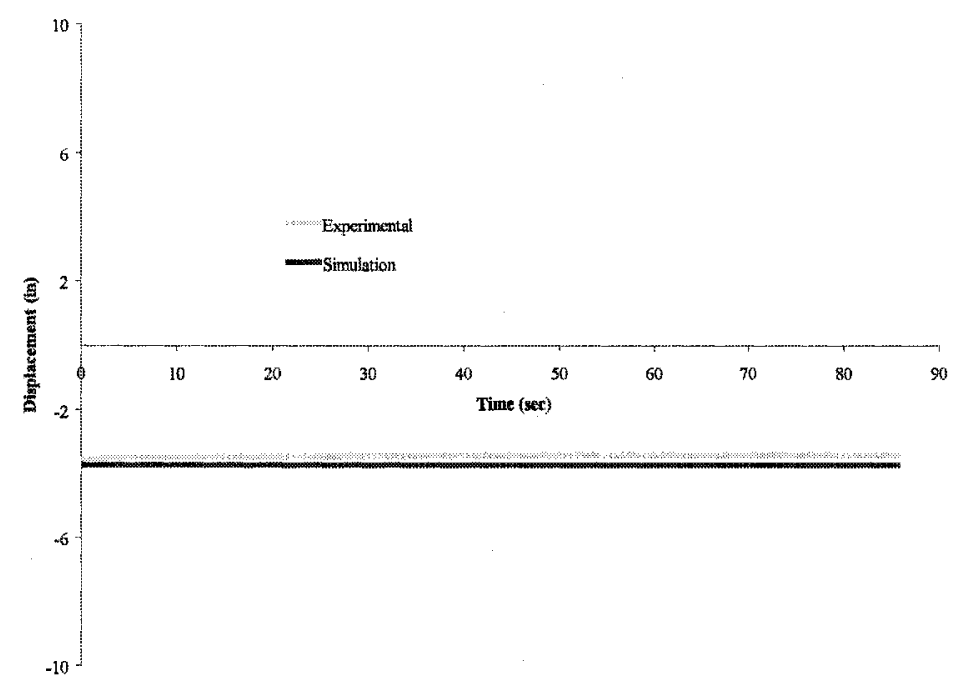

Figure 4.12: Comparison of the RSD claw y-position for Case 3.

The forces acting on the probe are shown in Figures 4.16 and 4.17. The new model shows a definite improvement in the $y$-direction force acting on the probe. The $\mathrm{x}$-direction forces do not correlate well with the measured experimental values. The $\mathrm{x}$-direction forces result from the components of lateral and longitudinal force produced by the tail wheel tires, as well as from the rolling resistance from the front tires. The tail wheel only contributes its lateral force to the probe's $\mathrm{x}$-direction force for short intervals since the force causes the castor to rotate to the orientation 


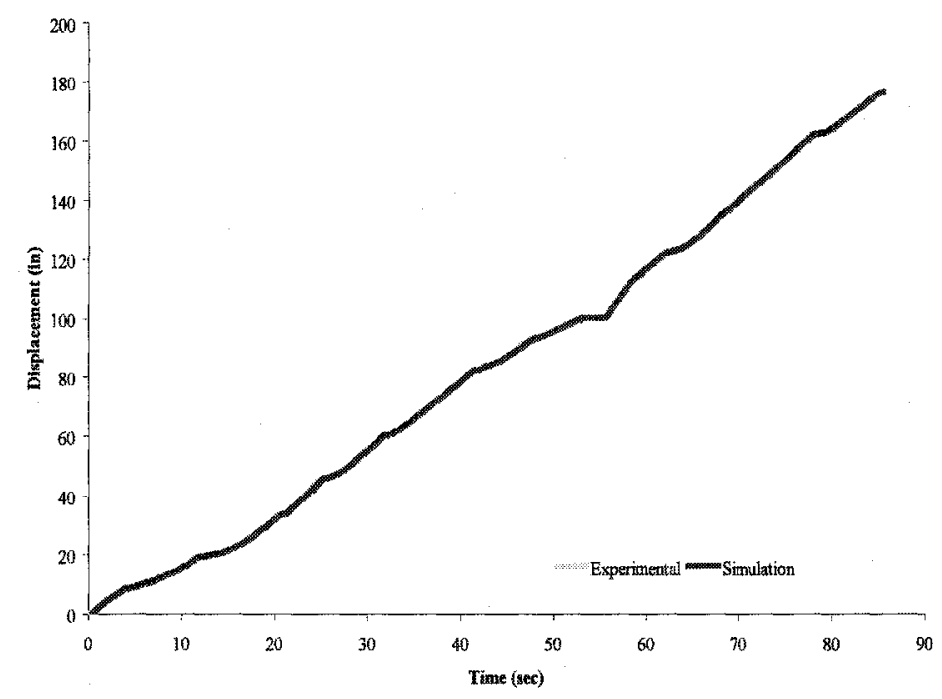

Figure 4.13: Comparison of the RSD claw x-position for Case 3.

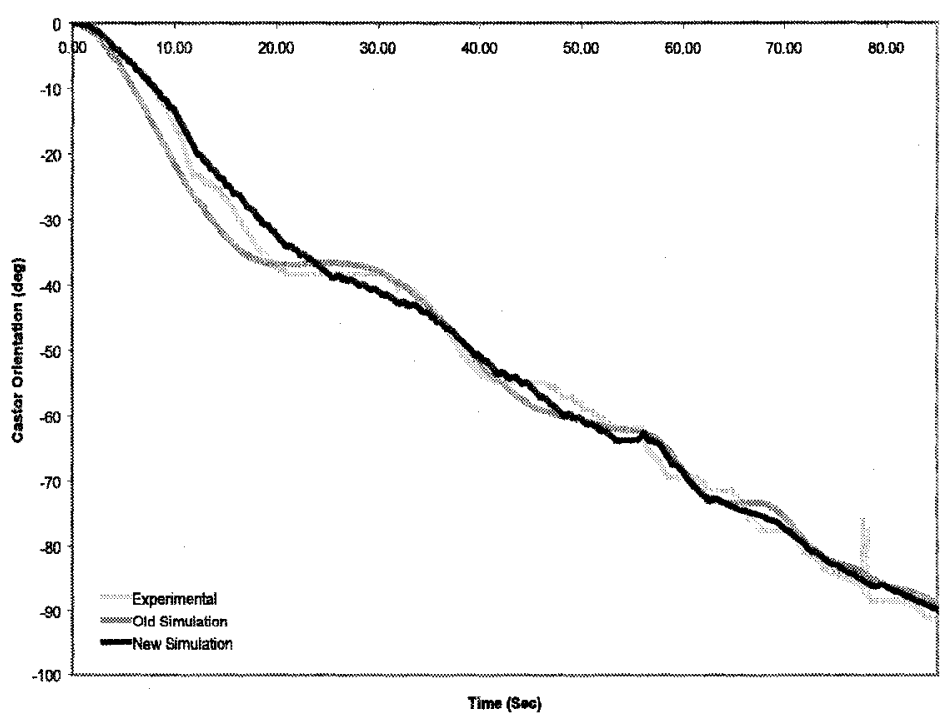

Figure 4.14: Comparison of the castor orientation for Case 3. 


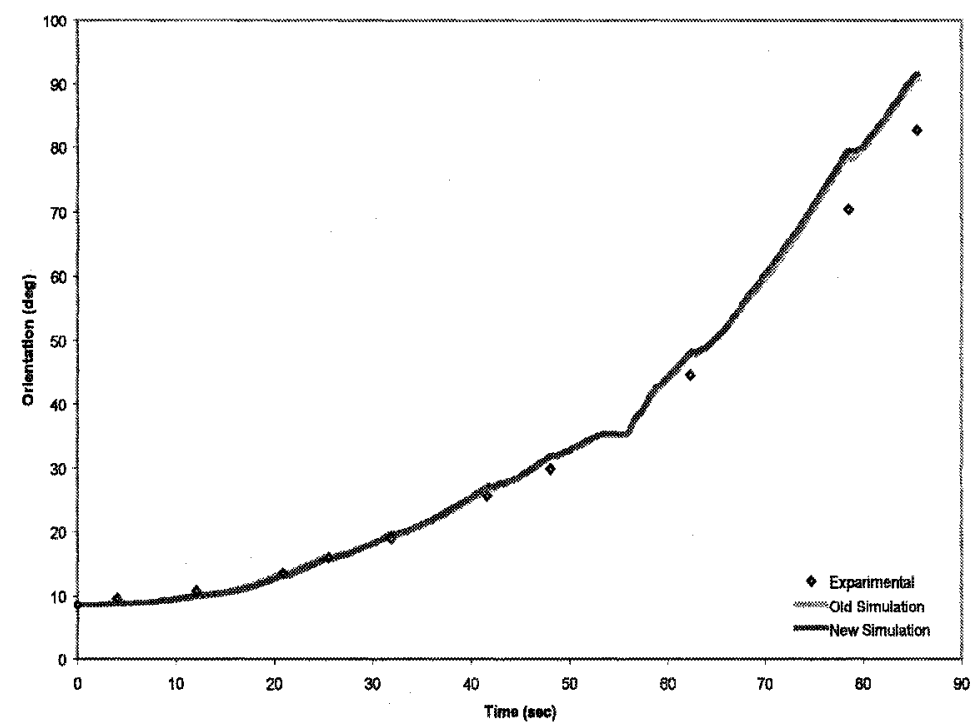

Figure 4.15: Comparison of the helicopter orientation for Case 3.

of least resistance. Therefore, the $\mathrm{x}$-direction probe force should mainly consist of rolling resistance, that is relatively small in magnitude since the tire is free to roll. The $\mathrm{x}$-direction shows forces of greater magnitude then those in the y-detection over some periods of time. This should not be the case.

The deflections of the right and left front tires are shown in Figures 4.18 and 4.19. The difference in deflection between the new tire model and the experiment can be attributed to initial conditions. However, the results of the y-direction forces on the probe correlate well. Therefore, the model is affecting the dynamics of the helicopter properly. There also may be a physical difference between the deflection of the single contact point of the transient tire model and the actual side deflection of the tire. The experiment measures the side wall deflection while the computational model simulates the deflection of the contact patch centre. The side wall is influenced by both the normal load and the lateral force, while the contact patch center is influenced by the lateral force only. The model is valid as long as the deflection made by the simulated tire produces the correct force. 


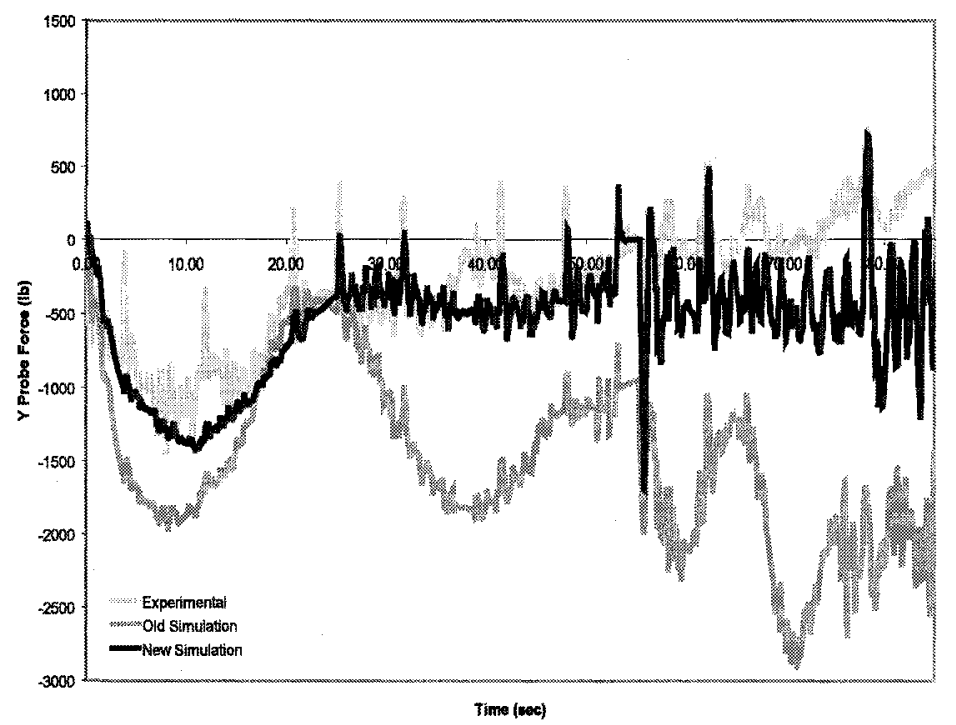

Figure 4.16: Comparison of the force acting on the probe in the DLTV's y-direction for Case 3.

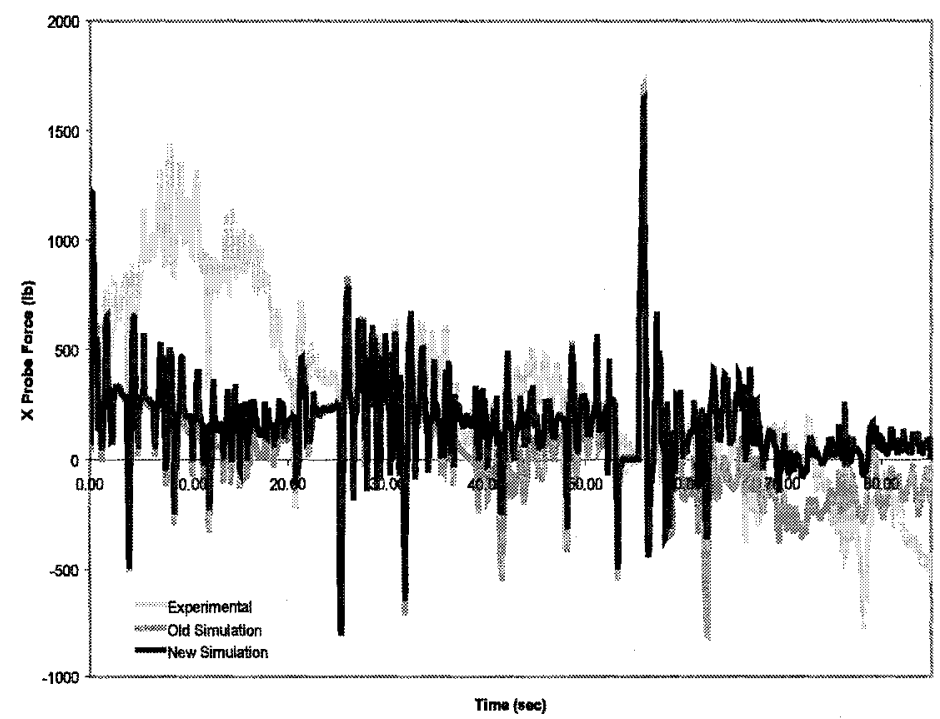

Figure 4.17: Comparison of the force acting on the probe in the DLTV's $\mathrm{x}$-direction for Case 3 . 


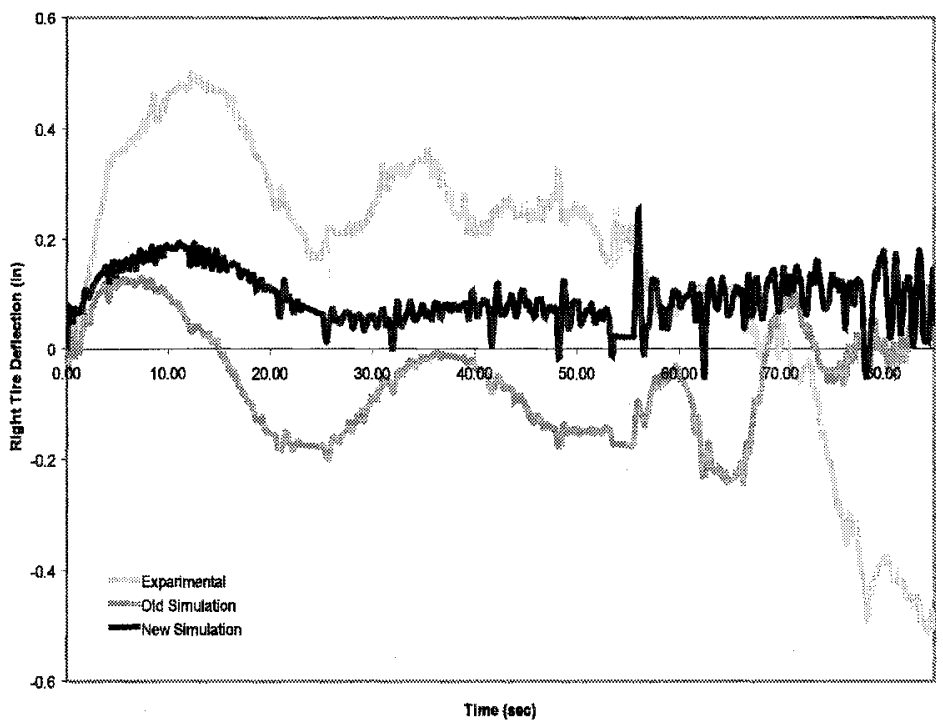

Figure 4.18: Comparison of the lateral deflection of the left tire for Case 3.

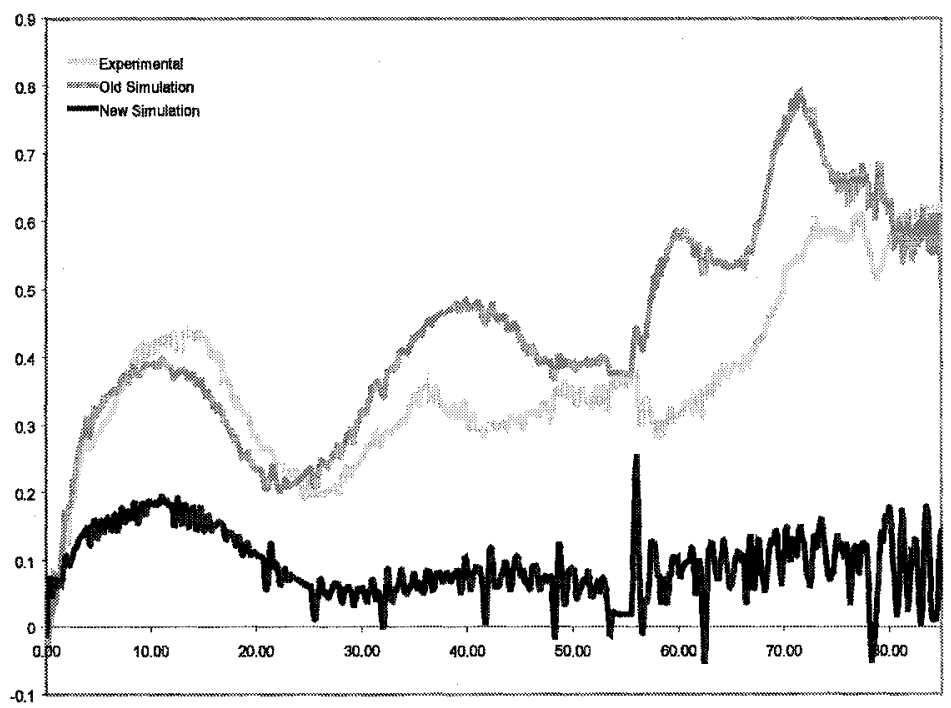

Figure 4.19: Comparison of the lateral deflection of the right for Case 3. 
Case 4: Yawed Back to 0 Degrees with Free Float

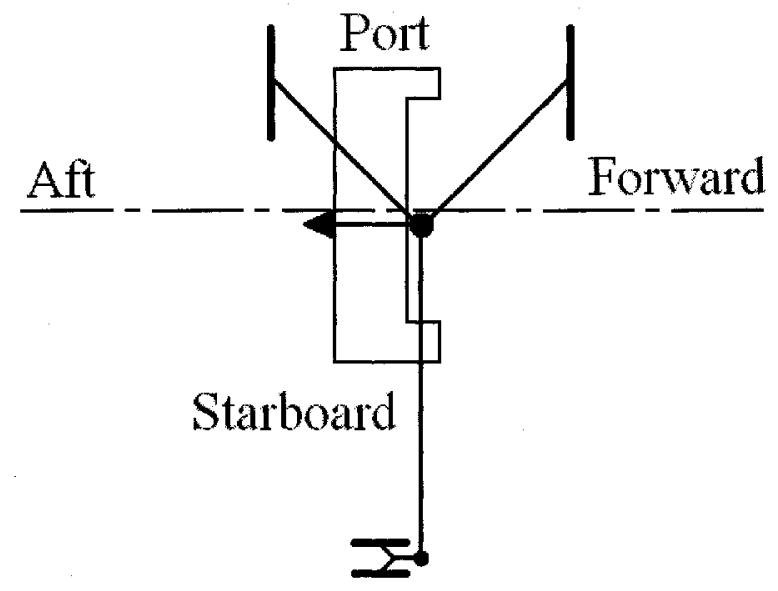

Figure 4.20: Initial position of DLTV and direction RSD is moved for Case 4.

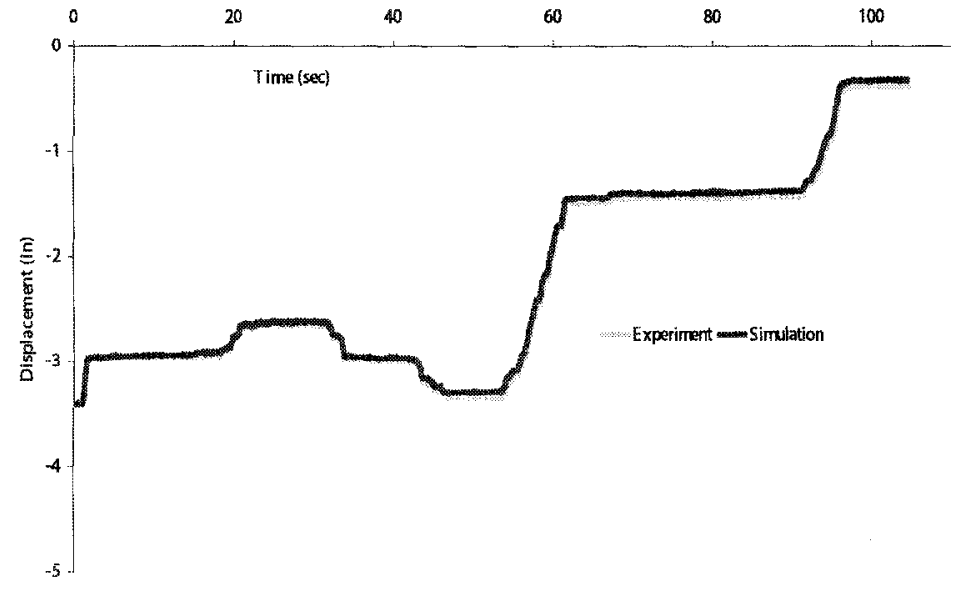

Figure 4.21: Comparison of the RSD claw y-position for Case 4.

The third test case had the DLTV start from the last position of the second test case. The DLTV is straightened from a position of $90^{\circ}$ until its longitudinal axis is parallel with the longitudinal axis of the ship. During the test, the DLTV was given an orientation as illustrated in Figure 4.20. Apparently, the castor wheel did not 


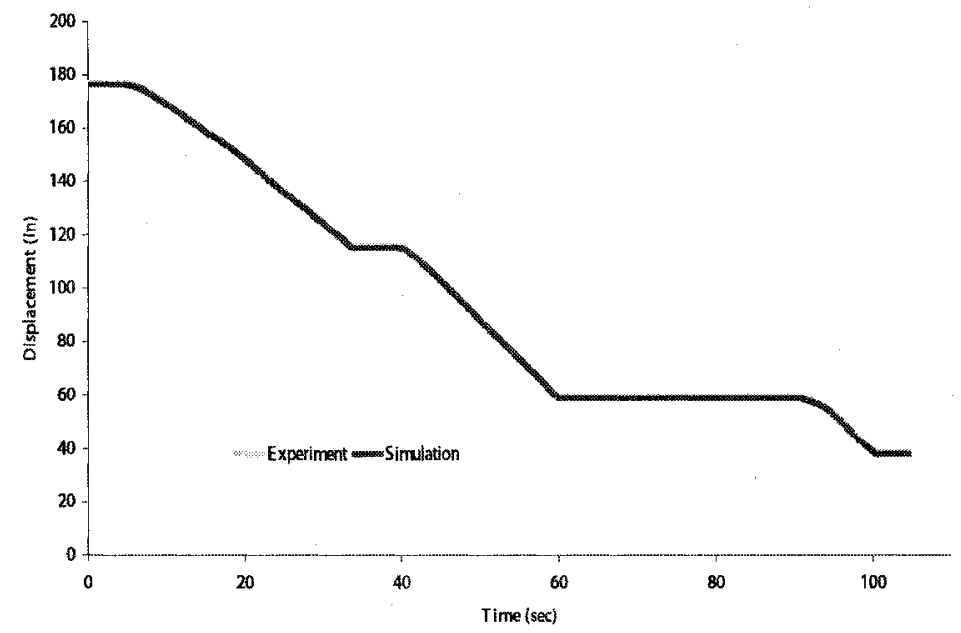

Figure 4.22: Comparison of the RSD claw x-position for Case 4.

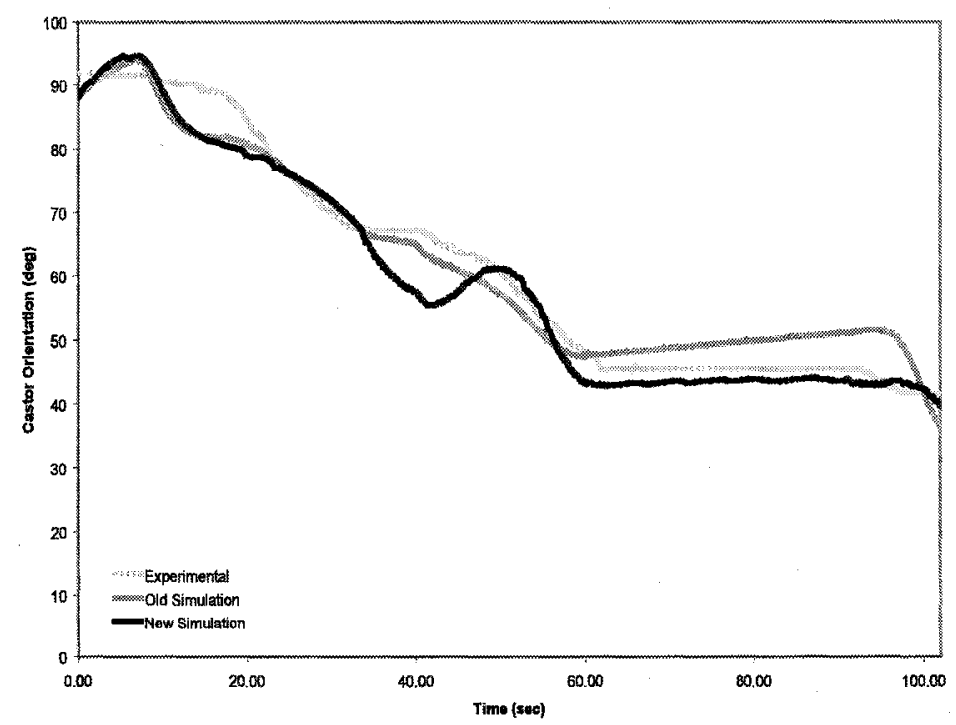

Figure 4.23: Comparison of the castor orientation for Case 4. 


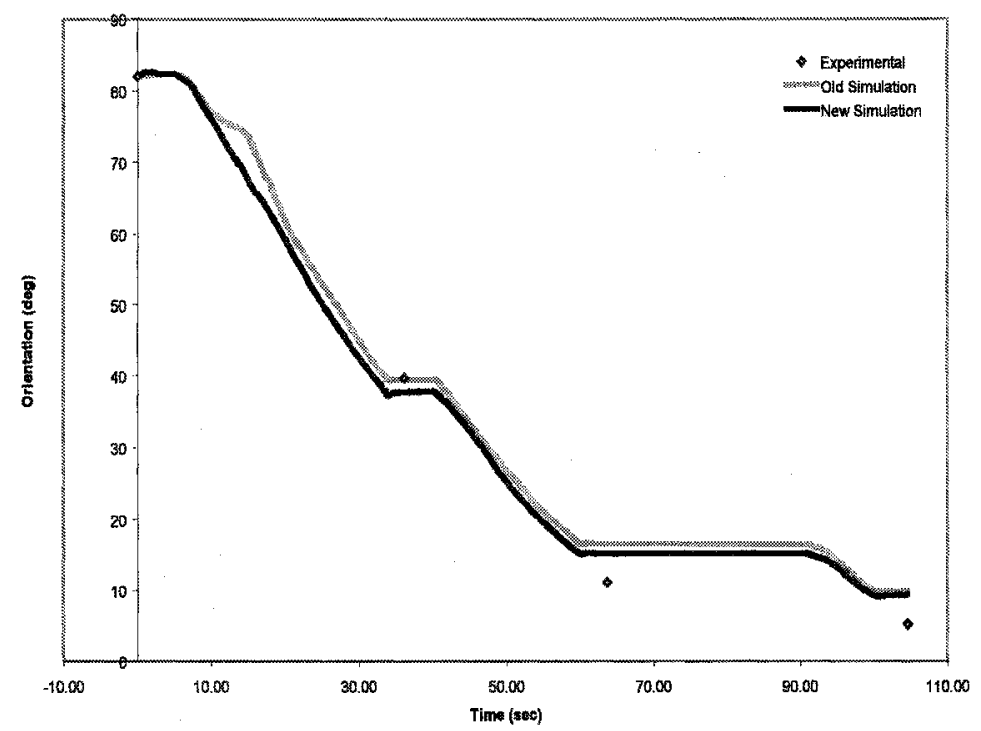

Figure 4.24: Comparison of the helicopter orientation for Case 4 .

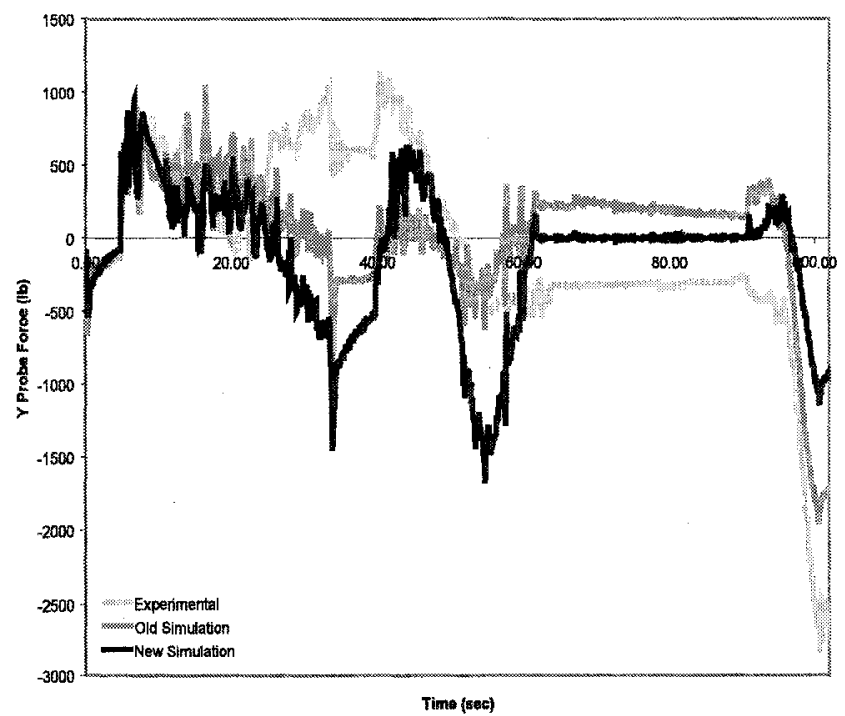

Figure 4.25: Comparison of the force acting on the probe in the DLTV's $\mathrm{y}$-direction for Case 4. 


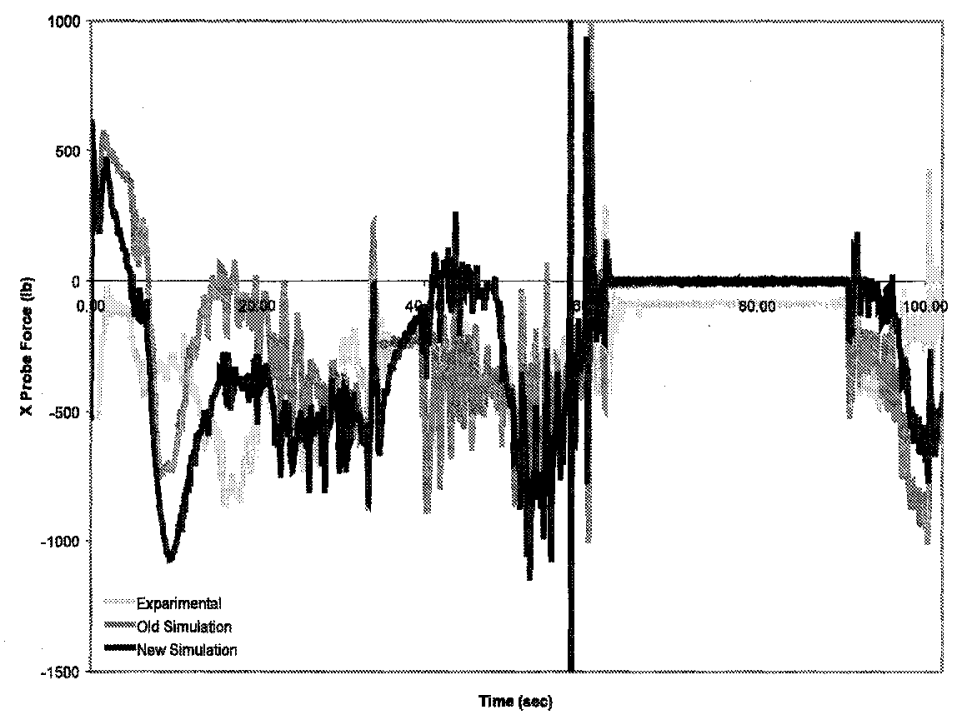

Figure 4.26: Comparison of the force acting on the probe in the DLTV's $\mathrm{x}$-direction for Case 4.

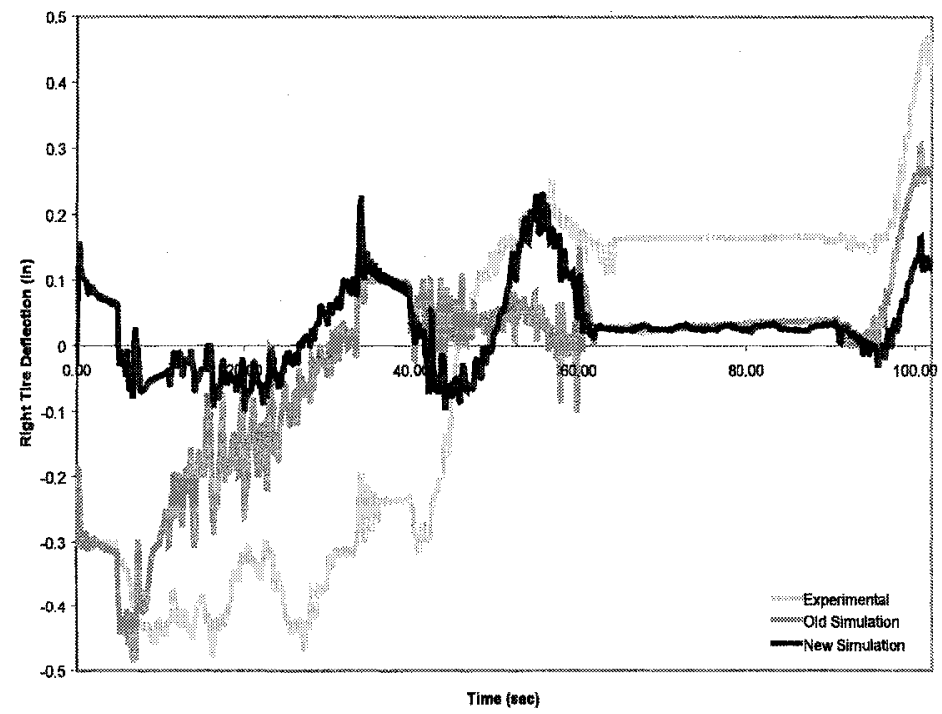

Figure 4.27: Comparison of the lateral deflection of the left tire for Case 4. 


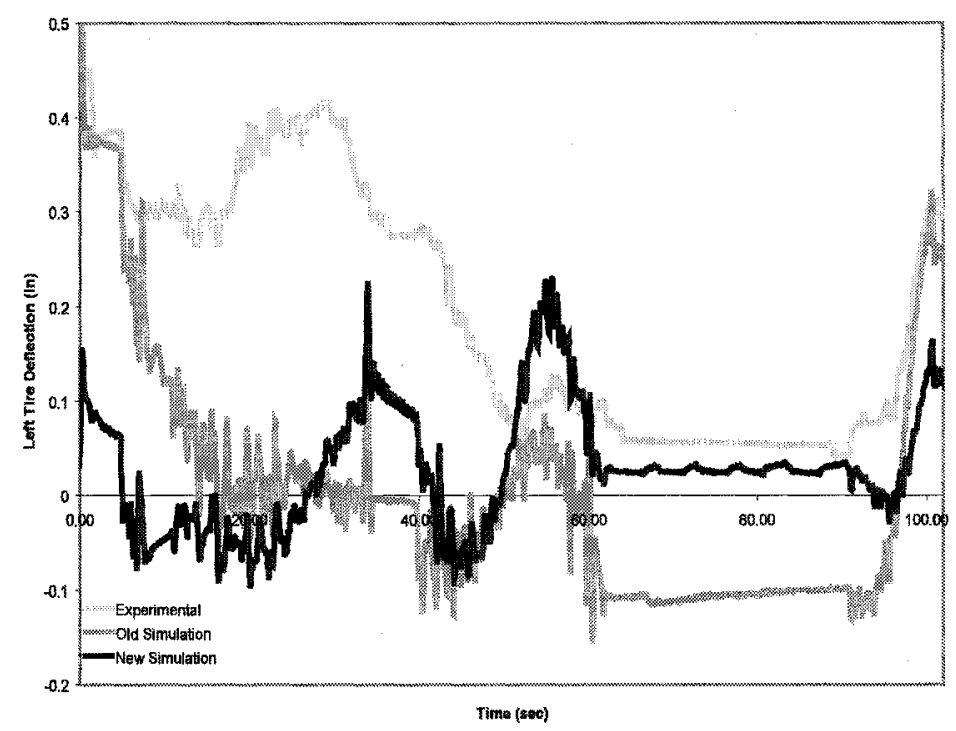

Figure 4.28: Comparison of the lateral deflection of the right for Case 4 .

rotate around $180^{\circ}$ as expected. According to Linn [1], an experienced operator of the ASIST system stated that this sometimes occurs. This is possible if the instantaneous center of velocity is positioned in such a way that would only allow pure tangential velocity of the castor assembly to occur. The castor joint would most likely have to experience an additional restricting moment as well that is not present in the simulation program. By design, the simulation includes a frictional moment that has zero magnitude at zero relative angular velocity to ensure stability of the numerical time propagation solution. This case will always be an unstable configuration in the simulation program. The results would be similar to those made by the castor wheel with an orientation of $180^{\circ}$ from that depicted in Figure 4.20. Therefore, the simulation will run with the tail wheel assembly pointed in the forward direction.

The comparison results for the probe position, castor orientation, helicopter orientation, probe forces, and front tire deflections are illustrated in Figures 4.21 through 4.28. The correlation between the results is fair with some major differences occurring in the middle of the test sequence. The differences are most likely due to the initial 
conditions on orientation and tire deflection.

\section{Case 7: Complex Manoeuvre}

The fourth test case represents a scenario where the helicopter begins with an offset and skewed position and orientation that requires the ASIST operator to straighten the helicopter's orientation out so that it may be traversed into the hanger. The scenario is a good test of the tire simulation's general performance. The results of the comparison study are presented as Figures 4.29 through 4.36. During the test the belt on the potentiometer measuring the castor orientation slipped and resulted in a period of no experimental castor rotation.

The new tire model contributed to a slight improvement in the $\mathrm{y}$ force results, illustrated in Figure 4.33. The results show that the new tire model chased the measured probe forces during the periods of 20 through 40 seconds and 50 through 190 seconds closer then the older tire model. The $\mathrm{x}$ force was also modelled with a greater accuracy then the older model with the exception of the last 50 seconds, as shown on Figure 4.34. The tire deflections behave in a similar fashion to the y force measurements, once again showing an overall improvement.

The results show that the new tire model contributes to an overall improvement in the simulation of the helicopter. In general, the new model has the greatest impact on improving the lateral force created by the tire. The simulation is able to replicate the dynamic behaviour of the helicopter well when it is undergoing a simple manoeuvre such as cases 1 and 3 . The greater the complexity of the manoeuvre the less accurate the tire dynamic behaviour is duplicated. This can be blamed in part by the need for greater accuracy in initial conditions placed on the simulation model. The tires will have initial deflections and forces that will affect their long term behavior. The helicopter and castor assembly orientation must also be set to their proper inial values 


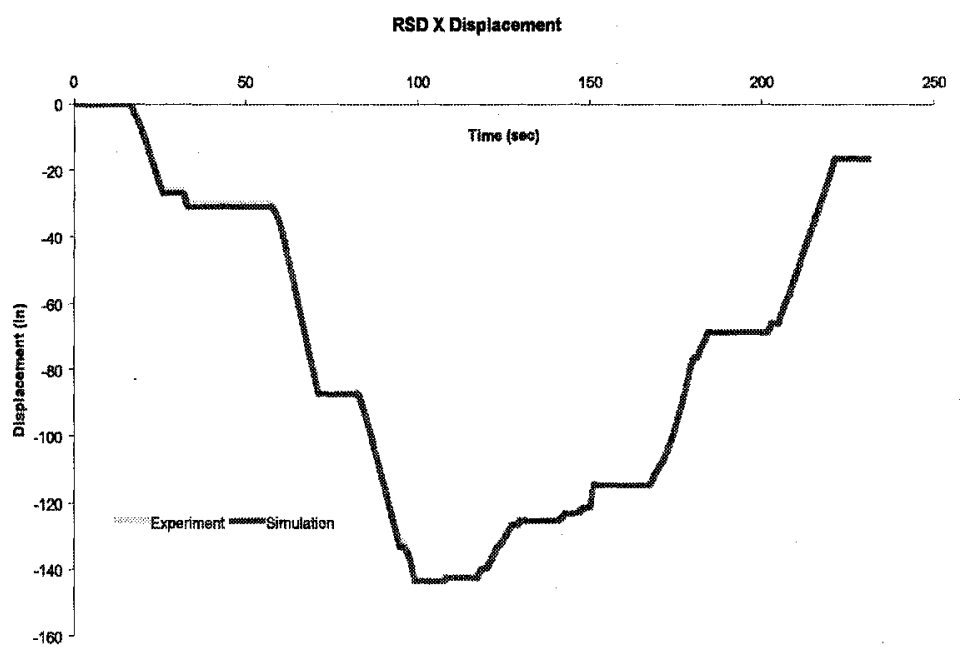

Figure 4.29: Comparison of the RSD claw y-position for Case 7.

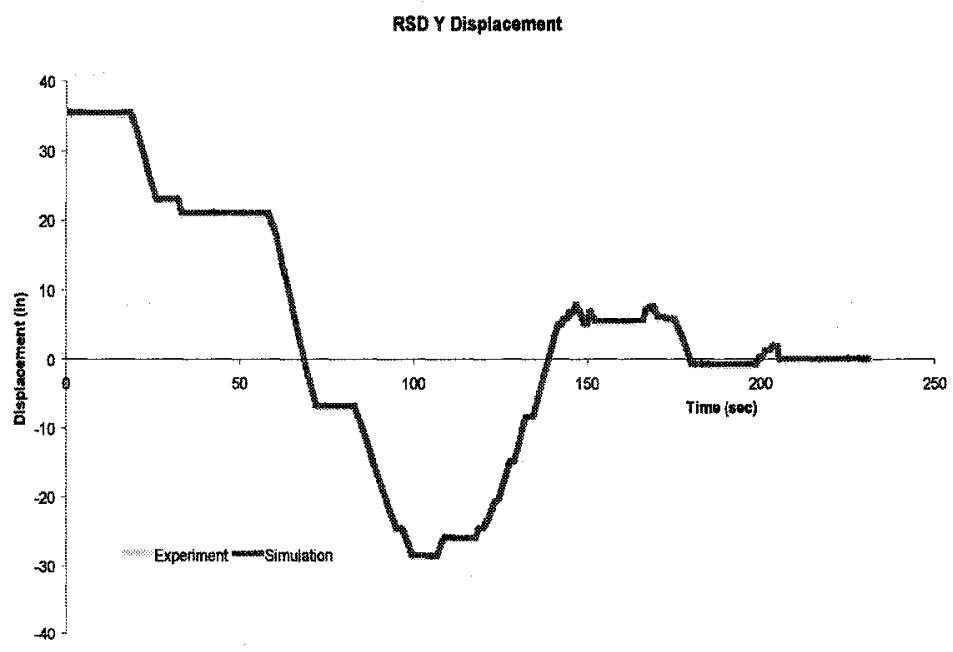

Figure 4.30: Comparison of the RSD claw x-position for Case 7 . 


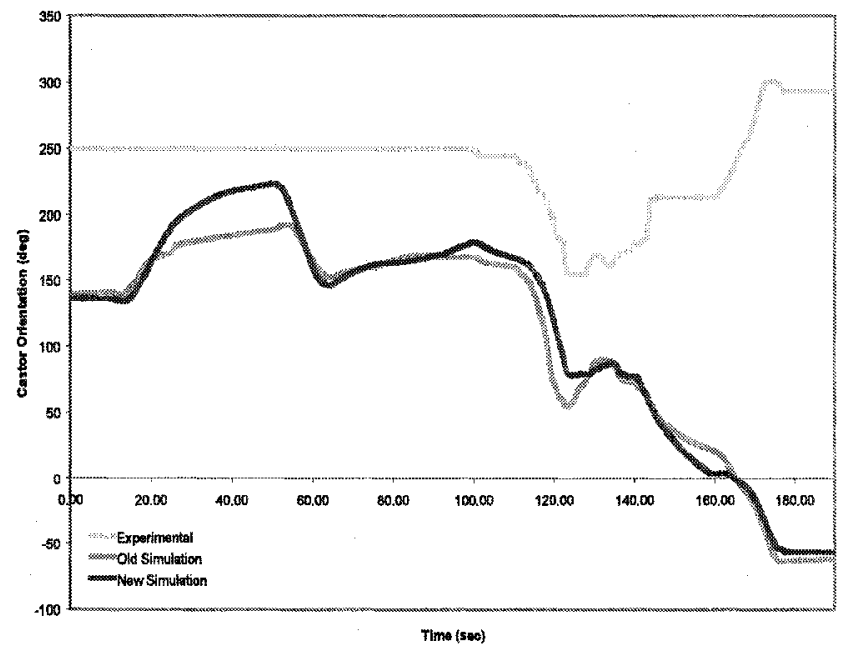

Figure 4.31: Comparison of the castor orientation for Case 7.

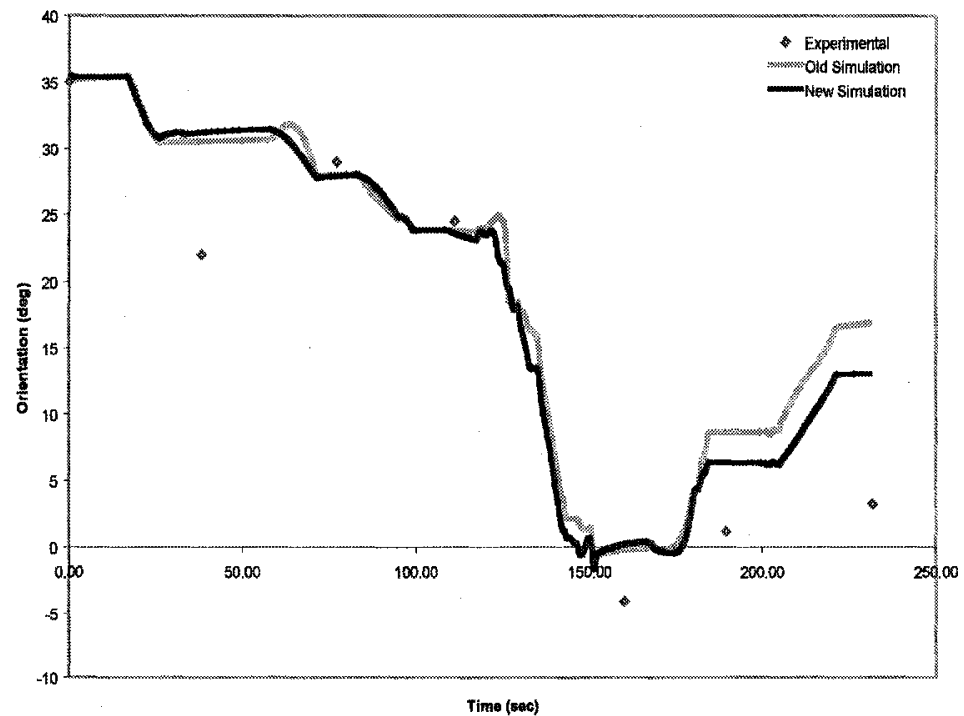

Figure 4.32: Comparison of the helicopter orientation for Case 7. 


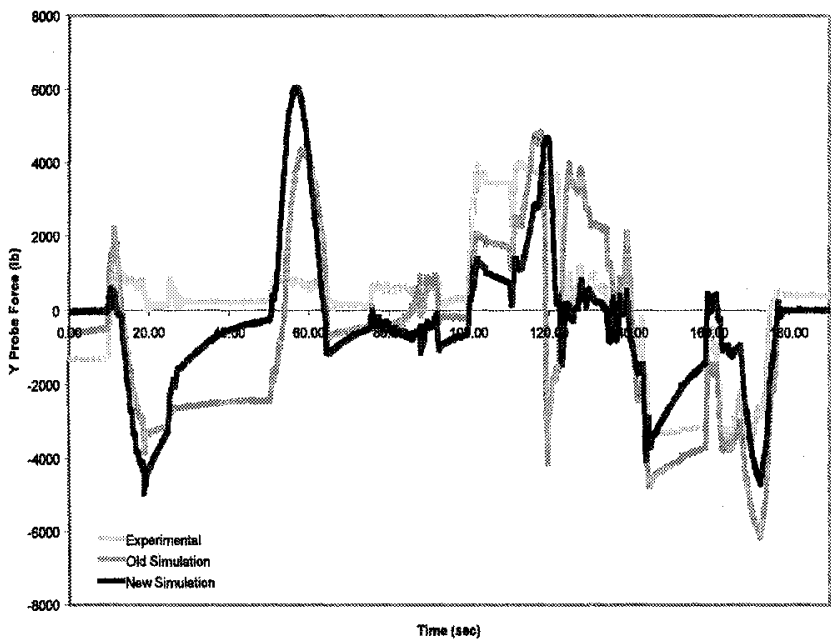

Figure 4.33: Comparison of the force acting on the probe in the DLTV's y-direction for Case 7.

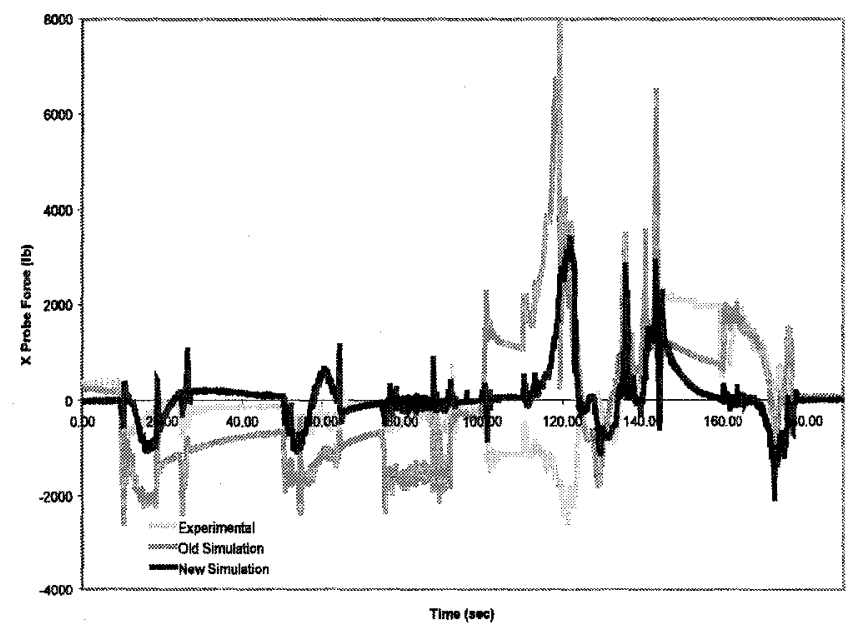

Figure 4.34: Comparison of the force acting on the probe in the DLTV's $\mathrm{x}$-direction for Case 7 . 


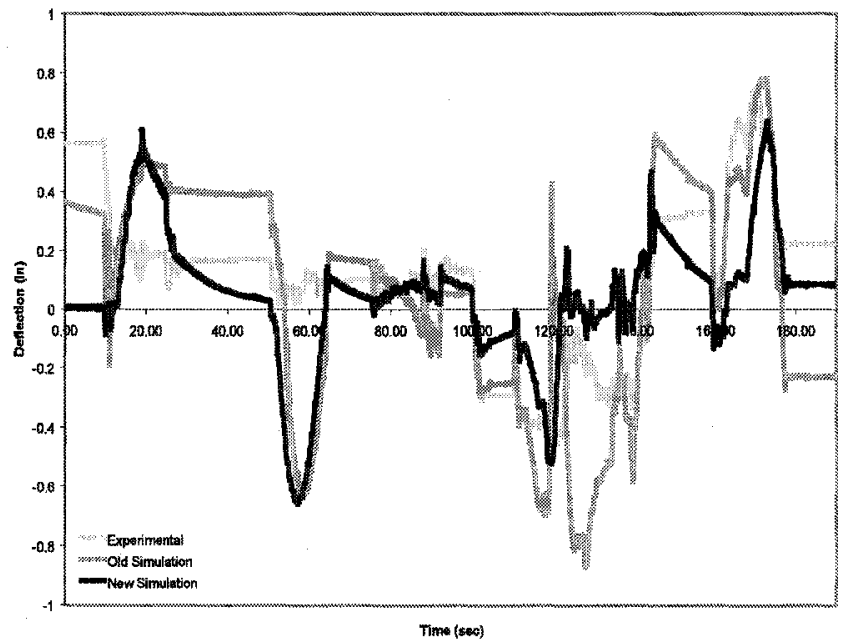

Figure 4.35: Comparison of the lateral deflection of the left tire for Case 7.

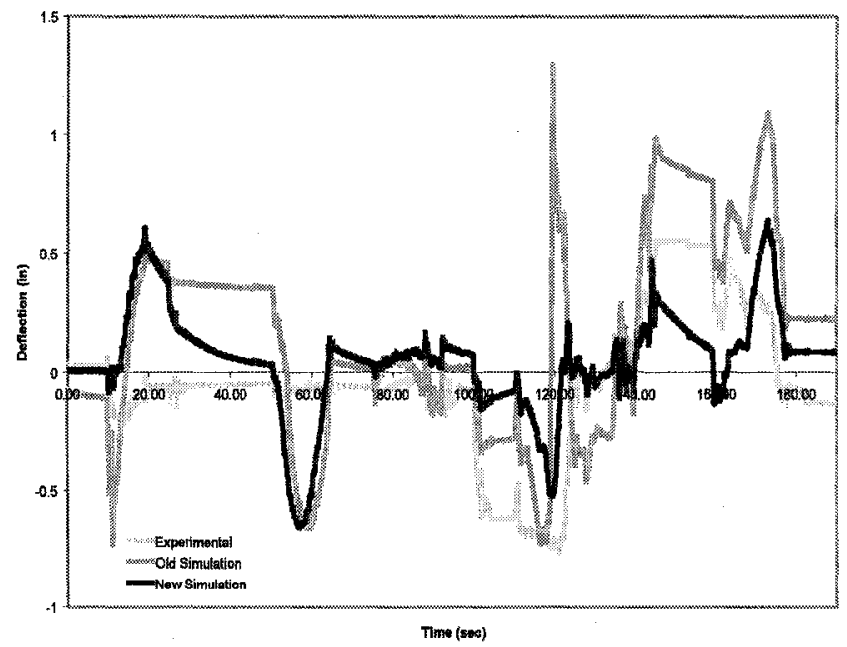

Figure 4.36: Comparison of the lateral deflection of the right for Case 7 . 
in order for the correct manoeuvre to occur. The complicated manoeuvres, such as case 7 , will be more susceptible to initial condition error. 


\section{Chapter 5}

\section{Discussion and Conclusion}

A new tire simulation was created to improve the dynamic behaviour of the HeliMan computer simulation. The new tire model can serve as an alternative to the Smiley and Horne tire model previously implemented. The overall dynamic behaviour improved through the implementation of the new tire model.

The new computational tire model was created by implementing the nonlinear version of the single contact point transient tire model presented by Pacejka. The nonlinearity of the mathematical model is due to the relaxation length varying with the transient slip angle. The problem is solved by obtaining the instantaneous relaxation length through the use of a steady state tire model. The string theory model relates the relaxation length to the slope of the lateral tire force versus slip angle curve. As a result, when the tire deformation increases the relaxation length decreases causing a faster transient response time.

The steady state tire model was created using Pacejka's Magic Formula tire model and Similarity Method tire model. The Magic Formula tire model consists of mathematical equations suited for matching the trends of tire behaviour due to longitudinal slip, lateral slip, turn slip, and camber inclination. The Similarity Method uses a 
nominal set of conditions of normal force, frictional coefficient, and various stiffness coefficients to model the tire behaviour due to changes in normal force and frictional coefficient.

The tire behaviour used in the steady state tire model was collected through tire testing of an aircraft tire closely resembling that used on the Sea King's tail wheels. Lateral force and aligning torque test data were collected by varying the tire's slip angle, camber inclination, and normal force. A nominal set of tire behaviour curves were modelled using the Magic Formula model. The results of the steady state tire model show that the Magic Formula curves match closely with the empirical data when modelling behaviour due to side slip and camber inclinations. The nominal data set was then altered through the use of the Similarity Method due to changes in normal force. The Similarity Method was successful in matching the results of the empirical data.

It was not possible to simulate the effects of turn slip using the tire test apparatus at Carleton University. In order to obtain feasible turn slip tire characteristics, a contact patch simulation program created by Pacejka called TreadSim was used. The contact patch was first modelled to simulate the results of the lateral forces and aligning moments to that of the tire test data. Once a match was obtained, the program was used to simulate the behaviour due to turn slip. The turn slip behaviour was then modelled through the use of the Magic Formula. The current version of the Magic Formula model can not exactly duplicate the effects of turn slip. However, the parameters of the Magic Formula were chosen to model the common operating points of turn slip and lateral slip closely.

The strengths of the new tire model lie in its flexibility to model desired tire behaviour. The tire model, created by the behaviour matching of one tire, had to be adapted to match the behaviour of the tires on the simulated Sea Hawk helicopter. 
This was done by making the assumption that the Sea Hawk's tires would behave similarly to the aviation tire used in the tire experiments. This is not completely true, since the Sea Hawk's tires have a higher pressure setting and are under a larger normal load. The front and castor tires also have a different ply rating in their construction. The ply, normal load, and pressure variation effect the tires stiffness. By altering the stiffness variable of the Magic Formula, the desired tire behaviour was obtained, demonstrated by the comparison of the HeliMan simulation with the DLTV experiments.

It is not known how well the new tire model will simulate changes in normal load. The DLTV experiment only consisted of one normal loading. The new model was calibrated to the normal loading of the Sea Hawk helicopter by changing the peak value coefficient of the Magic Formula model to the maximum allowable lateral force. The maximum lateral force is obtained by multiplying the normal force by the friction coefficient. This setting was then used in conjunction with the nominal settings of the steady state model created through the tire experiments. The simulation will mimic the changes in tire behaviour due to normal force in a similar fashion to the tire used in the tire experiment. To model the actual behaviour accurately, data concerning the cornering stiffness as a variable of normal loading is required. Altering the normal load on the DLTV and matching the tire simulation would be one way of obtaining this information. However, with multiple tires with different characteristics, this would be a difficult process. The best solution is to obtain actual data from explicit tire tests, hence the usual direct relationship between the amount of testing and the accuracy of the model.

Applying the new tire model to the HeliMan simulation mostly improved the lateral force generation. This can be seen by observing the force generated in the $y$ direction on the probe. The y-direction probe force is mostly due to tire lateral force 
with the exception of longitudinal force from the castor tires and the force generated by inertia.

The $\mathrm{x}$-direction probe force observed in the DLTV experiments is quite different from the forces generated by the simulation. There could be several reasons this is the case such as inadequate tire modelling, inaccurate inertial properties, or an error in data collection. For the case of tire modelling, most vehicle models simulate rolling resistance by offsetting the longitudinal force graph versus longitudinal slip. This creates a longitudinal force at zero longitudinal slip. The effect of rolling resistance is often quite small for vehicle simulation. However, for the case of a helicopter manoeuvring and traversing on a ship flight deck the tires undergo large quantities of lateral slip, at large normal pressures, while travelling relatively slowly. This might cause a longitudinal force that is significant and relative to the lateral force. An attempt was made to generate the longitudinal force by making it a ratio of lateral force prediction. This resulted in good x-direction probe force. However, all other dynamic characteristics were shifted away from their observed behaviour. Also, different ratios were required for the different test cases. As noted by Linn [1], it is possible that a misalignment of strain gauges could have effected the results. The shapes of the $\mathrm{x}$-direction probe force plots resemble the shape of $\mathrm{y}$-direction probe force plots in a fashion that suggest coupling between directions.

The new tire model underestimated the tire deflection observed from the results of the DLTV tests. One plausible explanation for this occurrence is the location of tire deformation. The tire model relates the position of the deformed contact patch centre to the position of the un-deformed contact patch centre. During the DLTV experiments, the tire deflection was measured on the side of the tire. The side wall of the tire will deform both due to normal load as well as lateral deflection. The deformation might not be as linear as the relation of the contact patch centre. 
The model could simulate the tire deflection closer by decreasing the lateral tire stiffness. However, this resulted in changes to the lateral force. Since the motion of the helicopter is governed by the forces, it was decided to model the force as closely as posible. The shape of the deflection is similar to that observed by the DLTV experiments with exceptions most likely due to the initial conditions of the tire deflection. One advantage to having the tire deflection modelled accurately is to predict when a tire might be unseated from its rim due to the tire over-deflecting. Since the tire behaviour is relatively accurate, a scaling ratio could be implemented to correct for the error.

The helicopter and castor orientation is well modelled by the simulation. One exception is the occurrence of the castor wheel not rotating when the castor wheel's longitudinal direction is pointed in the same tangential direction of the helicopter's rotation, such as the scenario in test case 3 . This is physically possible due to the near perfect tangential velocity of the tire due to the position of the castor's instantaneous centre, as well as perhaps some restricting moment in the castor joint which is not accurately modelled. In the simulation, this is an unstable configuration for this manoeuvre and the castor wheel will always rotate $180^{\circ}$.

The thesis demonstrates the use of the Magic Formula tire model with aviation tires, and increases the flexibility of the tire model implemented by HeliMan. The study of the helicopter manoeuvring and traversing on the ship flight deck allows for possible improvement of design in both the securing and traversing mechanism as well as helicopter landing gear design. It can also be used for training future operators and designing autonomous control of the manoeuvring and traversing of the helicopter in and out of the hanger. The new tire model is adaptable for use with a three dimensional helicopter/ship model where transient normal forces and camber inclinations will be included among the tire variables governing the response of the 
tires. 


\section{Bibliography}

[1] D.R. Linn. Development and validation of a planar on-deck helicopter manoeuvring simulation. Master's thesis, Carleton University, 2003.

[2] M. Hashimoto, H. Yoshinaga, H. Yoshikawa, N. Morikawa, T. Uekusa, and S. Asai. Three-dimensional finite element analysis of loaded tires. NEC Research $\&$ Development, 35(4):477-487, October 1994.

[3] H. J. Yu and H. Aboutorabi. Dynamics of tire, wheel, and suspension assembly. Tire Science and Technology, 29(2):66-78, April/June 2001.

[4] M. Shiraishi, H. Yoshinaga, A. Miyori, and E. Takahashi. Simulation of dynamically rolling tire. Tire Science and Technology, 28(4):264-276, October 2000.

[5] G. Anghelache F. Negrus and A. Stanescu. Finite element analysis and experimental analysis of natural frequencies. In F. Bohm and H.P. Willumeit, editors, Tyre models for vehicle dynamic analysis : proceedings of the 2nd International Colloquium on Tyre Models for Vehicle Dynamics Analysis, volume 27 of Vehicle System dynamics, pages 221-224, Delft, The Netherlands, February 1997. Swets \& Zeitlinger.

[6] H. B. Pacejka. Tire and Vehicle Dynamics. Society of Automotive Engineers, Inc., 400 Commonwealth, Warrendale, PA, U.S.A., 1st edition, 2002. 
[7] P. W. A. Zegelaar, S. Gong, and H. B. Pacejka. Tyre models for the study of inplane dynamics. In Proceedings of the 13th IAVSD Symposium on the Dynamics of Vehicles on Roads and on Tracks, volume 23 of Vehicle System Dynamics, pages 578-590, Sichuan, China, August 1993. Swets Publishing Services.

[8] G. H. Nybakken, R. N. Dodge, and S. K. Clark. A study of the dynamic tire properties over a range of tire constructions. Technical report, The University of Michigan, Ann Arbor, Michigan, U.S.A., March 1973. Prepared for Langley Research Center, NASA as report CR-2219.

[9] F. Montanaro G.Mastinu, S. Gaiazzi and D. Pirola. A semi-analytical tyre model for steaty and transient state simulation. In F. Bohm and H.P. Willumeit, editors, Tyre models for vehicle dynamic analysis : proceedings of the 2nd International Colloquium on Tyre Models for Vehicle Dynamics Analysis, volume 27 of Vehicle System dynamics, pages 2-21, Delft, The Netherlands, February 1997. Swets \& Zeitlinger.

[10] J. Zhou, J. Y. Wong, and R. S. Sharp. Multi-spoke, three plane tyre model for simulation of transient behaviour. Vehicle System Dynamics, 31(1):35-45, January 1999.

[11] H. B. Pacejka and I. J. M. Besselink. Magic formula tyre model with transient properties. In Proceedings of the 1997 2nd International Colloquium on Tyre Models for Vehicle Dynamic Analysis, volume 21 of Vehicle System Dynamics, pages 234-249, Berlin, Germany, Febuary 1997. Swets Publishing Services.

[12] M. Hoffman, E. Fischer, and B. Richter. The incorporation of tire models into vehicle simulations. In H. B. Pacejka, editor, Tyre models for vehicle dynamic analysis : proceedings of the 1st International Colloquium on Tyre Models for 
Vehicle Dynamics Analysis, volume 21 of Vehicle System dynamics, pages 4957, Delft, The Netherlands, October 1991. Swets \& Zeitlinger.

[13] Y. Tezuka, H. Ishii, and S. Kiyota. Application of the magic formula tire model to motorcycle maneuverability analysis. JSAE Review, 22(3):305-310, July 2001.

[14] M. T. P. van Slagmaat. Tire models in aircraft landing gear simulation. In Proceedings of the 1st International Colloquium on Tyre Models for Vehicle Dynamics Analysis, volume 21 of Vehicle System Dynamics, pages 108-115, Delft, Netherlands, October 1991. Swets Publishing Services.

[15] J.J.M. van Oosten and E. Bakker. Tire models in aircraft landing gear simulation. In Determintion of Magic Tyre Model Parameters, volume 21 of Vehicle System Dynamics, pages 19-29, Delft, Netherlands, October 1991. Swets Publishing Services.

[16] G. Leister. New procedures for tyre characteristics measurement. In F. Bohm and H.P. Willumeit, editors, Tyre models for vehicle dynamic analysis : proceedings of the 2nd International Colloquium on Tyre Models for Vehicle Dynamics Analysis, volume 27 of Vehicle System dynamics, pages 2-21, Delft, The Netherlands, February 1997. Swets \& Zeitlinger.

[17] R. F. Smiley and W. B. Horne. Mechanical properties of pneumatic tires with special reference to modern aircraft tires. Technical Report TR R-64, Langley Research Center, NASA, Langley Field, Virginia, U.S.A., 1960.

[18] C. R. Hogg. Simulation of Ground Handling of Taxiing Aircraft. PhD thesis, Department of Mechanical and Manufacturing Engineering, University of Brighton, Brighton, U.K., June 1993. 
[19] A. Self, C. Hogg, D. Pearce, and G. Kapadoukas. The simulation of taxiing and low flying aircraft. In F. Maceri and G. Iazeolla, editors, EUROSIM '92 Simulation Congress : proceedings of the 1992 EUROSIM Conference, pages 263-268, Capri, italy, September 1993. North-Holland.

[20] G. G. Kapadoukas, A. W. Self, and C. J. Harris. Flight simulation: Eigenvalue analysis during taxiing. In Chinni, editor, Proceedings of the Military, Government and Aerospace Conference.

[21] B. Mallock, C. J. Harris, C. R. Hogg, and D. Jones. Trimming of flight simulation ground models. Proceedings of the Institution of Mechanical Engineers, Part G: Journal of Aerospace Engineering, 211(2):91-102, 1997.

[22] R.H. Daugherty. A study of mechanical properties of modern radial aircraft tires. Technical Report NASA/TM-2003-212415, Langley Research Center, NASA, Langley Field, Virginia, U.S.A., 2003.

[23] A. Higuchi and H.B. Pacejka. The relaxation length concept at large wheel slip and camber. In F. Bohm and H.P. Willumeit, editors, Tyre models for vehicle dynamic analysis : proceedings of the 2nd International Colloquium on Tyre Models for Vehicle Dynamics Analysis, volume 27 of Vehicle System dynamics, pages 2-21, Delft, The Netherlands, February 1997. Swets \& Zeitlinger.

[24] Song-Tao Chen. Analysis of the Tractive Performance of Pneumatic Tires Over Soft Terrain. PhD thesis, Carleton University, 1993.

[25] Yeun-Chung Wu. Handling of Multiaxle All-Wheel-Drive Off-Road Vehicles. PhD thesis, Carleton University, 2000. 
[26] Standard for the required level and measurment of coefficient of friction on flight decks. North Atlantic Treaty Organization Standardardization Agreement, (STANAG N 1278 (Edition 2)), February 2005.

[27] Coating system, non-skid, for roll or spray applicaiton (metric). Performance Specification, (MIL-PRF-24667A(NAVY)), August 1992. 


\section{Appendix A}

\section{Test Equipment and Calibration}

Figures A.1 through A.4 show the calibrations of both Lebow dual component load cells used to gather the forces on the tires. The load cells have an $\mathrm{x}$-direction capacity of $1000 \mathrm{lb}$ and a y-direction capacity of $500 \mathrm{lb}$. Each channel of the load cells were tested using an axial load testing machine in the structural laboratory at Carleton University. All of the channels were in proper working order with only a minute amount of instrument error due to the load versus voltage relationship. 


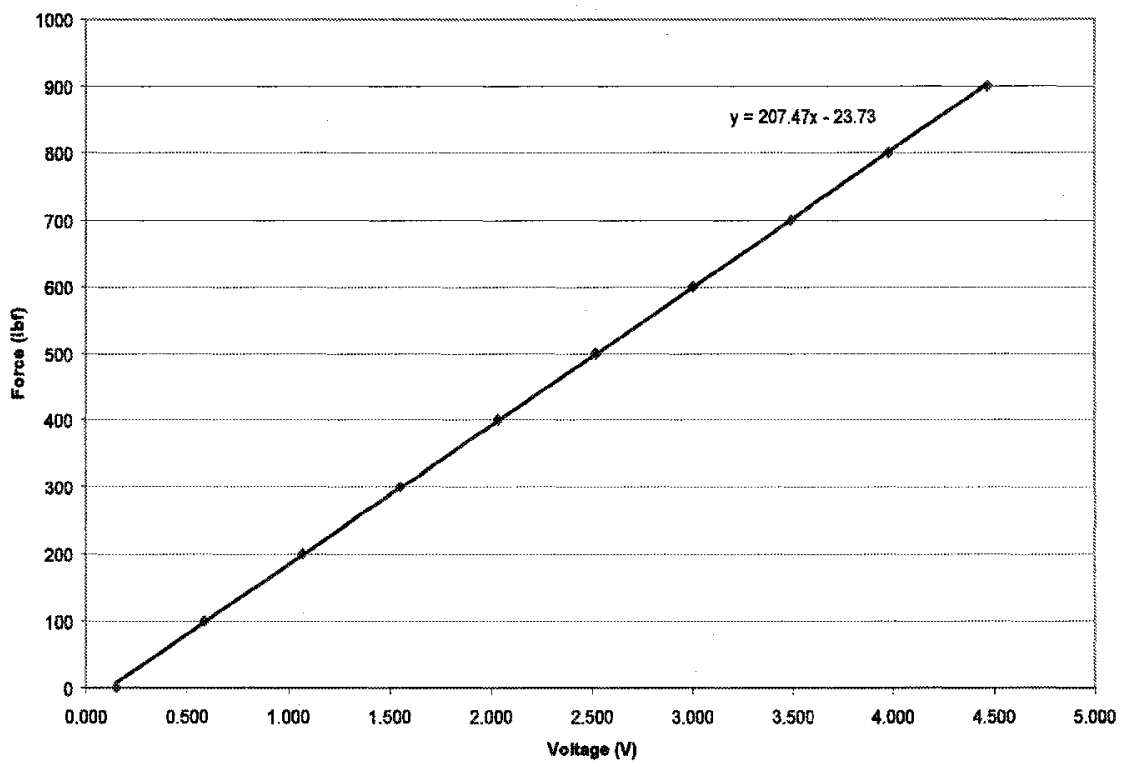

Figure A.1: Calibration of load cell $7745 \mathrm{x}$-force.

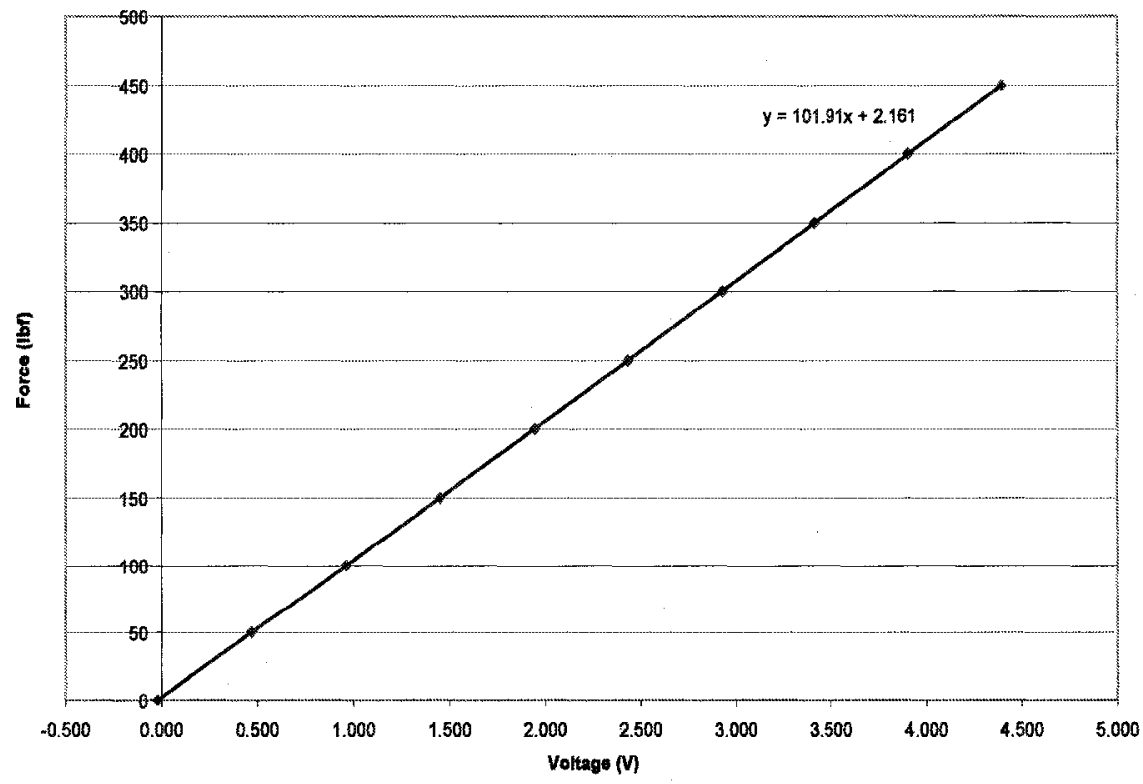

Figure A.2: Calibration of load cell 7745 y-force. 


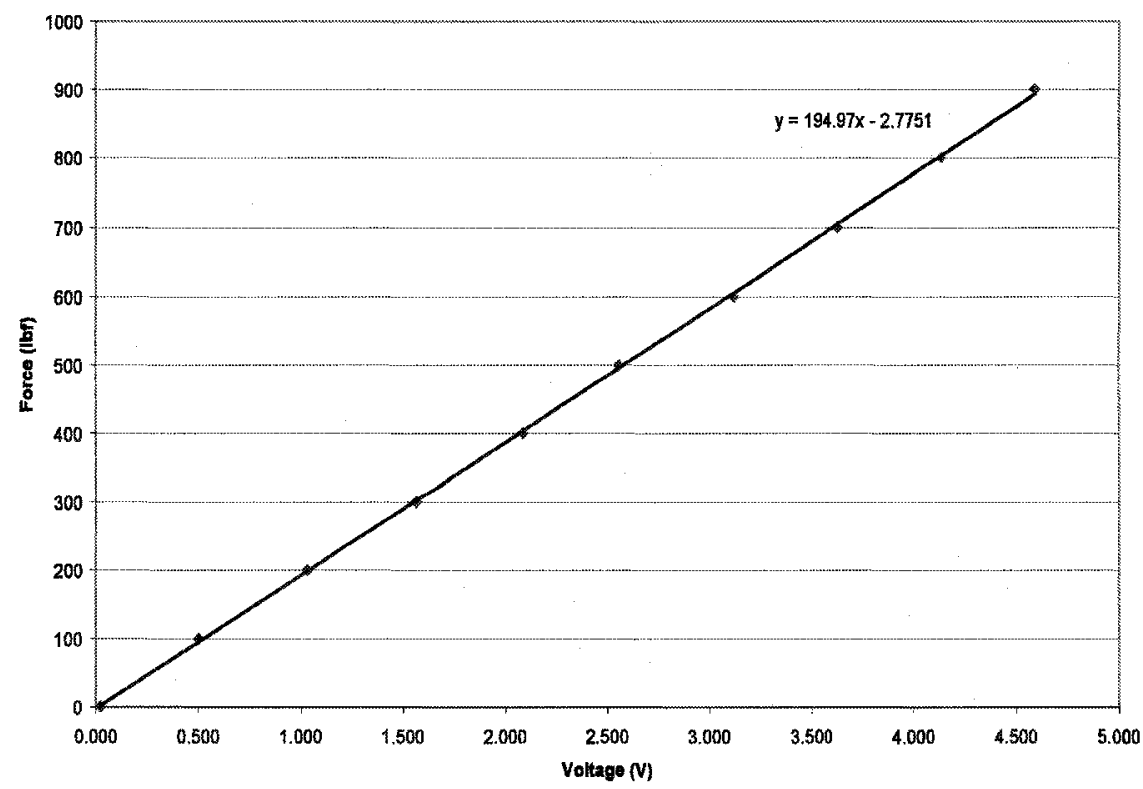

Figure A.3: Calibration of load cell $7700 \mathrm{x}$-force.

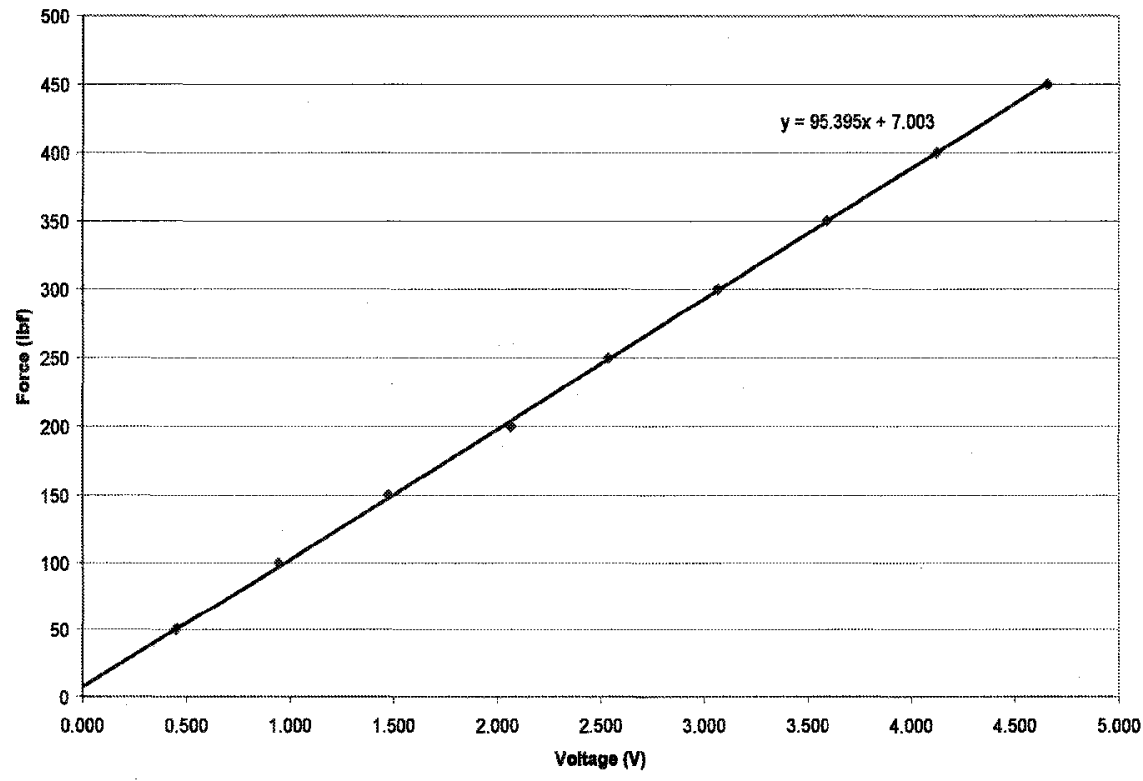

Figure A.4: Calibration of load cell 7700 y-force. 